\title{
NISTIR 4369
}

\section{System Requirements Analysis for the \\ U.S. Army Rock Island Arsenal Tool Management System}

Simon P. Frechette

Charles R. McLean

U.S. Department of Commerce National Institute of Standards and Technology Center for Manufacturing Engineering Factory Automation Systems Division Gaithersburg, MD 20899

Prepared for:

The U.S. Army Rock Island Arsenal U.S. Department of Defense

Washington, DC 20301

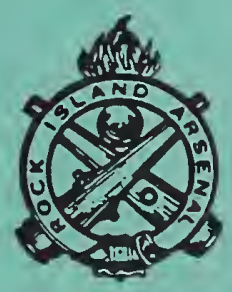





\section{NISTIR 4369}

\section{System Requirements Analysis for the \\ U.S. Army Rock Island Arsenal Tool Management System}

Simon P. Frechette

Charles R. McLean

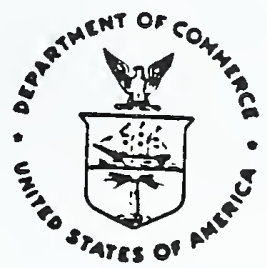

U.S. Department of Commerce

National Institute of Standards and Technology

Center for Manufacturing Engineering

Factory Automation Systems Division

Gaithersburg, MD 20899

Prepared for:

The U.S. Army Rock Island Arsenal

U.S. Department of Defense

Washington, DC 20301

August 1990

U.S. Department of Commerce

Robert A. Mosbacher, Secretary

National Institute of Standards and Technology John W. Lyons, Director 


\section{.}


Certain commercial equipment, instruments, or materials are identified in this paper in order to adequately specify the experimental procedure. Such identification does not imply recommendation or endorsement by the National Institute of Standards and Technology or the U.S. Army Rock Island Arsenal, nor does it imply that the materials or equipment identified are necessarily the best available for the purpose. 
List of Figures $\ldots \ldots \ldots \ldots \ldots \ldots \ldots \ldots \ldots \ldots \ldots \ldots \ldots \ldots$

Acknowledgements $\ldots \ldots \ldots \ldots \ldots \ldots \ldots \ldots \ldots \ldots \ldots \ldots \ldots \ldots \ldots \ldots \ldots$

Executive Summary $\ldots \ldots \ldots \ldots \ldots \ldots \ldots \ldots \ldots \ldots \ldots \ldots \ldots \ldots \ldots$

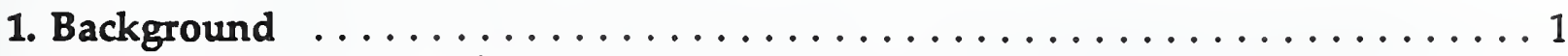

1.1 A Brief Overview of RIA Manufacturing $\ldots \ldots \ldots \ldots \ldots \ldots \ldots$

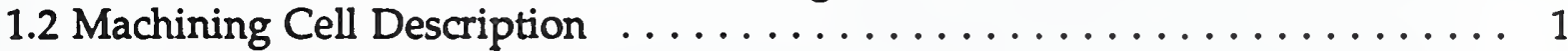

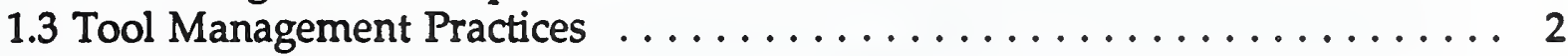

1.4 Goals and Expected Benefits $\ldots \ldots \ldots \ldots \ldots \ldots \ldots \ldots \ldots \ldots \ldots$

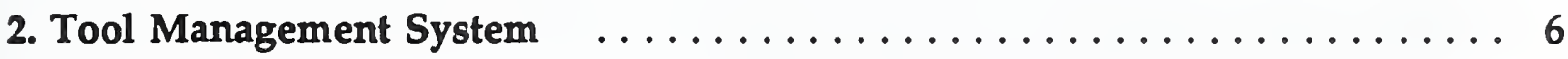

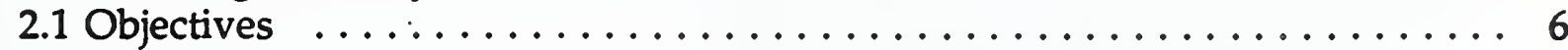

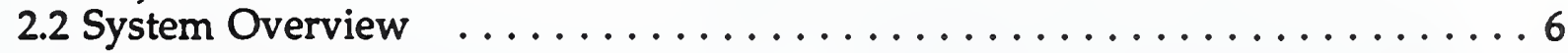

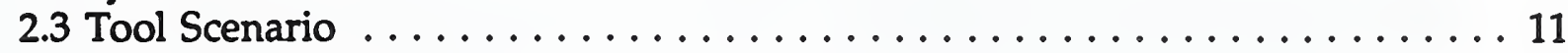

2.4 Introduction to Subsystem Requirements . . . . . . . . . . . . 15

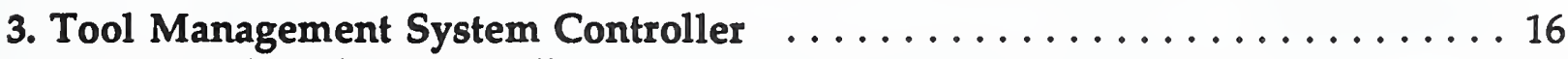

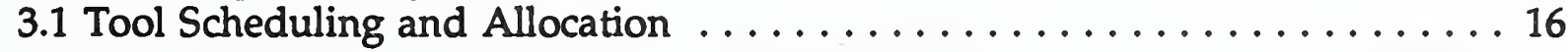

3.2 Tool Dispatching . . . . . . . . . . . . . . . . . . 24

3.3 Tool-life Monitoring, Analysis, and Assignment . . . . . . . . . 26

4. Tool Room .................................. 30

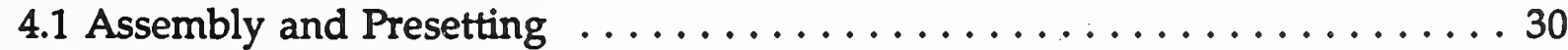

4.2 Component Inventory Management $\ldots \ldots \ldots \ldots \ldots \ldots \ldots \ldots$

4.3 Assembly Storage, Issue and Receipts . . . . . . . . . . . . . . . 39

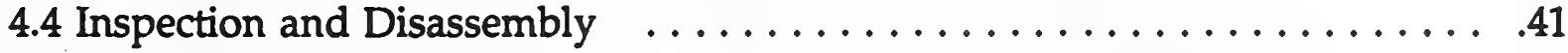

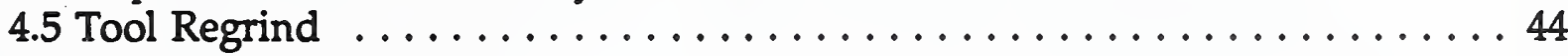

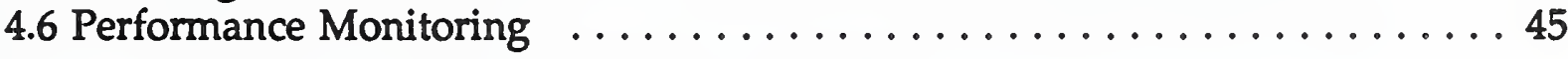

5. Tool Assembly Storage and Delivery $\ldots \ldots \ldots \ldots \ldots \ldots \ldots \ldots$

5.1 Automated Tool Assembly Delivery ... . . . . . . . . . . . . . . . . 49

5.2 Automated Storage and Retrieval of Tooling . . . . . . . . . . . 52

5.3 Manual Handling and Storage $\ldots \ldots \ldots \ldots \ldots \ldots \ldots \ldots \ldots$

5.4 System Controller . . . . . . . . . . . . . . . . . . 58

6. Tool Tracking and Data Collection $\ldots \ldots \ldots \ldots \ldots \ldots \ldots \ldots \ldots \ldots$

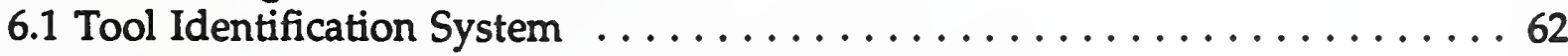

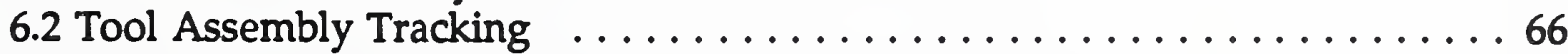

6.3 Tool Monitoring at Machine Tools . . . . . . . . . . . . . . . . . 69

6.4 Tool Data Collection (Freeze Frame) $\ldots \ldots \ldots \ldots \ldots \ldots . \ldots . \ldots 73$ 
7. Tool Management Upgrades to the Existing Cell $\ldots \ldots \ldots \ldots \ldots \ldots$

7.1 Tool Handling by Robot(s) . . . . . . . . . . . . . . . . . 76

7.2 Loading and Unloading of Tool Magazines ................ 83

7.3 Tool Storage in Buffer Areas . . . . . . . . . . . . . . . . 85

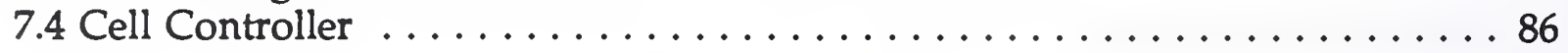

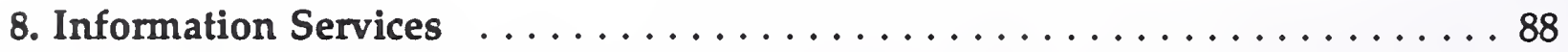

8.1 Data Base Management . . . . . . . . . . . . . . . . . . 88

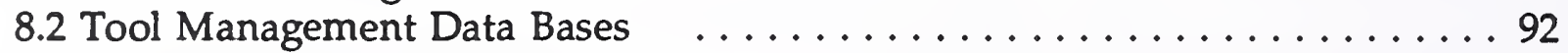

8.3 Communications Network .......................... 94

8.4 Peripheral Devices - Terminals, Printers, Readers, etc. . . . . . . . . . . 97

9. CIM System Links . . . . . . . . . . . . . . . . . . . . . 99

9.1 Data Services and External Databases ..................... 99

9.2 Distributed Numerical Control System . . . . . . . . . . . . . . . . . 100

9.3 Network Bridge or Gateway . . . . . . . . . . . . . . . . 100

9.4 Workbench User Interface . . . . . . . . . . . . . . . . . . 100

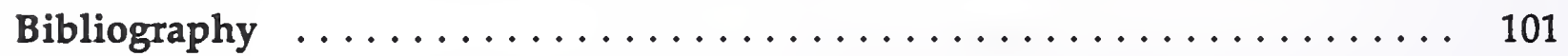

Glossary of Terms ............................ 104 


\section{List of Figures}

Figure 1-1. The existing flexible machining cell at RIA

Figure 2-1. Major functional areas of tool management

Figure 2-2. Component modules of the proposed tool management system

Figure 2-3. An example tool management scenario

Figure 3-1. Allocation strategy: bulk exchange

Figure 3-2. Allocation strategy: shared tooling

Figure 3-3. Allocation strategy: migration

Figure 3-4. Allocation strategy: resident tooling

Figure 4-1. Tool component and assembly flow within the tool room

Figure 4-2. Data flow within the tool room

Figure 4-3. Example terminal display of tool setup specification

Figure 5-1. Automated tool assembly delivery system

Figure 5-2. Manual tool assembly delivery

Figure 5-3. Block diagram of proposed automated system

Figure 5-4. Block diagram of proposed manual system

Figure 6-1. Tool identification system

Figure 6-2. Freeze Frame real-time data collection system

Figure 7-1. Gantry robot configured with tool buffer racks

Figure 7-2. Gantry robot configured with carousel tool storage

Figure 7-3. Monorail robot configured with carousel tool storage

Figure 8-1. Tool management databases 


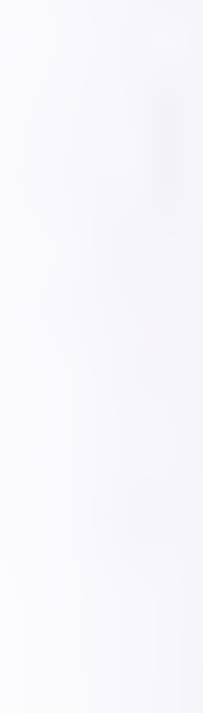




\section{Acknowledgements}

To our colleagues at Rock Island, we would like to express our thanks for the tremendous support that they gave us. We would especially like to recognize the technical contributions of Dr. Jack Moriarty and Mr. Robert Daligee, without whose efforts this project probably would not have gotten off the ground. We would also like to thank Dr. Richard Kalkan, the management lead at RIA on this project.

We appreciate the support of Mr. Fred Dearborn, the Civilian Executive Assistant of the Rock Island Arsenal for helping to establish the collaborative relationship with the National Institute of Standards and Technology. We would also like to recognize the support of Steve Robinson, Manager of the REARM/CIMS Project for the time that he and his staff spent with us. We especially thank Jerry Hatch and Steve Harris for their technical contributions to this effort.

At NIST, we would like to thank Dr. Pierre Kacha for his technical contributions and editing of sections of this document. Finally, we would like to recognize Mark Gerules from the University of Kansas for his help in preparing illustrations for this document. 


\section{Executive Summary}

The purpose of this document is to outline requirements for implementing an automated tool management system for flexible machining cells at The U.S. Army Rock Island Arsenal (RIA) in Illinois. The Rock Island Arsenal is currently conducting an extensive manufacturing modernization program. A flexible machining cell system (FMCS) has recently been installed in the facility as an "island of automation." Procurements of other "islands of automation" and manufacturing information systems are expected in the future.

Tool management, as it applies to these automated systems, is one area that RIA has identified for additional modernization activities. The engineers at the Rock Island Arsenal and the National Institute of Standards and Technology (NIST) recognize that a consistent and integrated implementation of tool management is critical to the Army's manufacturing program. With its current work in real-time tool performance data collection (Freeze Frame), adaptive control of cutting processes, and now in automated tool management systems, RIA is establishing itself as a leader in this field.

Tool management is not a single, simple activity - it is comprised of a complex and pervasive set of functions. The failure of a manufacturing system to perform these functions properly (i.e., get the right tool to the right machine at the right time) can often bring production processes to a halt. For example, some of the issues associated with tool management include:

* Tool preparation often requires design, fabrication, and assembly - tools and fixtures are usually fabricated for use on a single job. The normal design, process planning, and fabrication steps must be carried out just to create the tools to work on that job. Ideally, the tooling data generated and used in these steps should be shared between a number of different systems.

* Tool crib management is a major inventory control problem - a typical fabrication shop maintains an inventory of thousands of cutting tools, tool holders or adapters, and fixtures. Systems are needed to manage the tool inventory within the tool crib, record issues and receipts of tools to shop personnel, and track tool movements within an automated manufacturing systems. Carrying costs for unnecessary tool inventory are high. Unused or obsolete tooling must be identified and removed from inventory.

* Tool planning is both a material requirements planning and a scheduling problem - tool requirements are directly linked to the production schedule. Purchase orders for perishable tooling must be released to 
vendors with sufficient lead time to ensure timely delivery to the facility. Similarly lead times must be considered for assembly and tool setting operations within the crib to ensure that machines do not sit idle while their operators await tooling.

* Effective use of production resources means that jobs must be able to be reassigned to different machines - the reassignment of jobs due to bottlenecks or machine failures necessitates the migration of supporting tools. Due to use of different adapters or setup requirements for a new machine, the assignment of a job to a new machine may mean that the tools will have to be returned to the tool crib for reassembly and resetting.

* Economic use of tooling means making effective use of tool-life -accurate techniques or systems for estimation or measurement of remaining tool-life are essential. Tool-life enters into the tool selection process, determining gross tool requirements for individual jobs, planning the replenishment of tooling at machining centers for large jobs, evaluating the performance of individual vendor products, and determining when to scrap or rework individual tools.

Although tool management systems with limited capabilities have been developed, they are basically stand-alone systems which are difficult to integrate into a larger factory information system.

As World War II vintage machine tools have been replaced with more modern numerically controlled (NC) machines and flexible manufacturing systems, the compatibility problem has become more and more evident. For example, the programs that are used to produce parts on newer machines will not run on older models from the same manufacturer. Manufacturing systems constructed by different vendors are highly incompatible. For the most part they have closed, proprietary architectures. Research in automated manufacturing at NIST is directed at developing open system architectures that would provide satisfactory answers to the above questions.

Because industry has only recently begun to look more closely at the tool management problem, very little information about automated tool management is publicly available. Few documents are available which are helpful in defining requirements and specifications for automated tool management. No commercial systems are known to exist which perform all of the functions outlined in this document. This study documents joint efforts by NIST and RIA staff to develop an open architecture for tool management at the Rock Island manufacturing facility. 
Previous architectures for automation developed by NIST focus on defining key interface points between the major subsystems of an automated manufacturing facility. NIST scientists and engineers believe that an open architecture, based upon publicly defined interfaces, can be used to construct integrated factories from proprietary components. During the assembly of the Automated Manufacturing Research Facility (AMRF) test bed, NIST researchers chose to integrate hardware and software products from a number of different system vendors in order to substantiate this belief about open architectures. The integration process has taught NIST scientists and engineers a great deal about how to identify, specify and implement system interfaces. Due to the lack of interface standards for factory automation systems and the competitive government procurement process, it is likely that the same architecture and interface issues that have concerned researchers at NIST will be critical at U.S. Army manufacturing facilities.

A tool management architecture would serve as a reference model, defining the major subsystems and the interfaces that exist between them. It could provide a basis for planning the future integration of tool management functions into Department of Defense (DOD) manufacturing systems. Some of the functions, critical to effective tool management, that must be addressed by an architecture include:

* tool/fixture design

* tooling rationalization

* process planning, tool design and tool selection

* tooling list/bill of material preparation

* requirements planning and scheduling

* assembly, setting and kitting

* tool assembly identification and tracking

* tool room inventory control

* tool storage

* delivery of tools

* tool transfer within FMS systems

* alternate tool migration strategies

* tool wear monitoring

* estimation of remaining cutter life

* tool breakage detection

* imminent tool failure sensing

* tool reconditioning

* job cost accounting

* system simulation and analysis

* tooling procurements and vendor performance analysis 
Unfortunately, current "turnkey" commercial implementations of these functions by different system vendors are not likely to be compatible. Systems that perform these functions must be capable of interacting with each other, with other factory systems, and with people performing manual activities.

The functions listed above are specified in detail within this document according to the following categories:

* the tool management system controller (Section 3)

* the tool room (Section 4)

* tool assembly storage and delivery (Section 5)

* tool tracking and data collection (Section 6)

* tool management upgrades to the existing cell (Section 7)

* information services (Section 8)

* CIM system links (Section 9)

A bibliography of suggested reading on tool management topics and a glossary of terms can be found at the end of this document.

With the completion of this requirement analysis, a major step has been made towards defining what automated tool management should be at RIA. The next steps are already underway which will result in the development of:

* detailed system design specifications

* simulation and emulation models of the proposed system

* database definitions

* module interface specifications

When this specification phase is over, it will be necessary to assess the technical and economic feasibility of implementing the proposed system. If it is indeed feasible, the system would mostly likely be constructed using off-the-shelf or custom-built products, and the services of commercial system integrators. 


\section{Background}

\subsection{A Brief Overview of RIA Manufacturing}

The Rock Island Arsenal has been serving the defense needs of the nation for over 100 years. It is located in the central United States on a 946 acre island in the Mississippi River. The FY 1988 Annual Report for the Rock Island Arsenal describes its three distinct missions:

* Manufacture of artillery, gun mounts, recoil mechanisms, small arms and spare parts;

* Perform tool set and basic issue item fabrication assembly for AMCCOM and other AMC sub-MACOMS, and

* Provide administrative, logistical and facility support services for RIA, HQ, AMCCOM, and a host of tenant activities.

The RIA manufacturing facilities have been undergoing a major modernization as a part of the \$220 million REARM project. Some of the features of the project included the construction of a building for a high density, automated storage and retrieval system and the installation of a flexible machining cell. Other improvements included the construction of an electroplating, welding and founding facility. A forge shop was also expanded and some existing buildings were rehabilitated.

The purpose of this requirements analysis is to identify tool management extensions for the flexible NC machining cell that was installed by Project REARM.

\subsection{Machining Cell Description}

The Kearney and Trecker machining cell handles parts weighing from ten to several hundred pounds. It is comprised of two Model $800 \mathrm{~K} \& \mathrm{~T}$ horizontal machining centers which are serviced by a pallet shuttle mechanism. The pallet shuttle system has nine pallet storage positions and three fixturing stations. The tool magazines on each machining center hold 68 tool assemblies. RIA staff expect that typical jobs will use 30 to 80 different tools and will require several hours of cutting per setup. The existing machining cell is illustrated in Figure 1-1.

The cell is currently in the process of being upgraded to a total of seven machining centers and one coordinate measuring machine. The new cell will have four K\&T Orion 2300 and three K\&T Model 800 horizontal machining centers. Each Orion 
2300 machine will be serviced by a six position carousel pallet shuttle. Seven of the machining centers will be used for production and one will be used for prove-out. All machining centers will use RS-494 Binary Cutter Location (BCL) data format. A distributed numerical control (DNC) system will deliver BCL programs to the cell controller which will download them to the machining centers.

Two DeVlieg tool setting stations have already been installed in the tool room. The stations will be directly connected to the cell in the near future to transfer tool offset data.

Specifications for an overhead tool delivery robot are currently in development. Under a grant from NIST, the University of Illinois is performing simulation analyses of possible robot tool delivery system configurations.

\subsection{Tool Management Practices}

Current tool management practices, within the RIA machine shop, are typical of those generally found in industry. Several tool rooms (i.e., tool cribs) are strategically located around the RIA facility. The tool rooms stock tool components, maintain computerized inventory data on tooling, initiate stock replacements when inventory levels reach reorder point, assemble and preset tools to specification, store assembled tools prior to release to shop personnel, and regrind cutters that can be reused.

Within the tool room, component inventory is maintained in drawers and racks on shelves. Stock levels of each component are maintained in a computerized inventory control system that was implemented by RIA staff. The system maintains key data about each tooling component, usage, reorder point levels, and suppliers. Reports are generated for inventory verification and procurement initiation when reorder points are reached. Orders for replacement stock are placed through RIA's regular procurement channels. The RIA procurement department has some of the responsibility for vendor selection.

Part programmers design tools and prepare tool setup specifications. Standard setups are called out by number in the header section of the NC program. Traveler packages are prepared for each production job. The traveler packages contain shop order data, NC tapes, tool lists, and tool setup specifications. Standard tool setup specifications may be marked with dimensions and other requirements that are specific to the individual job. Shop foremen review traveler packages for pending jobs and provide production tooling requirements to the tool room manager, who schedules tool setup activities. It is normal practice for the tool room to receive 48-hour advanced notice of impending tool requirements. Under normal conditions, the tool room is staffed on a 


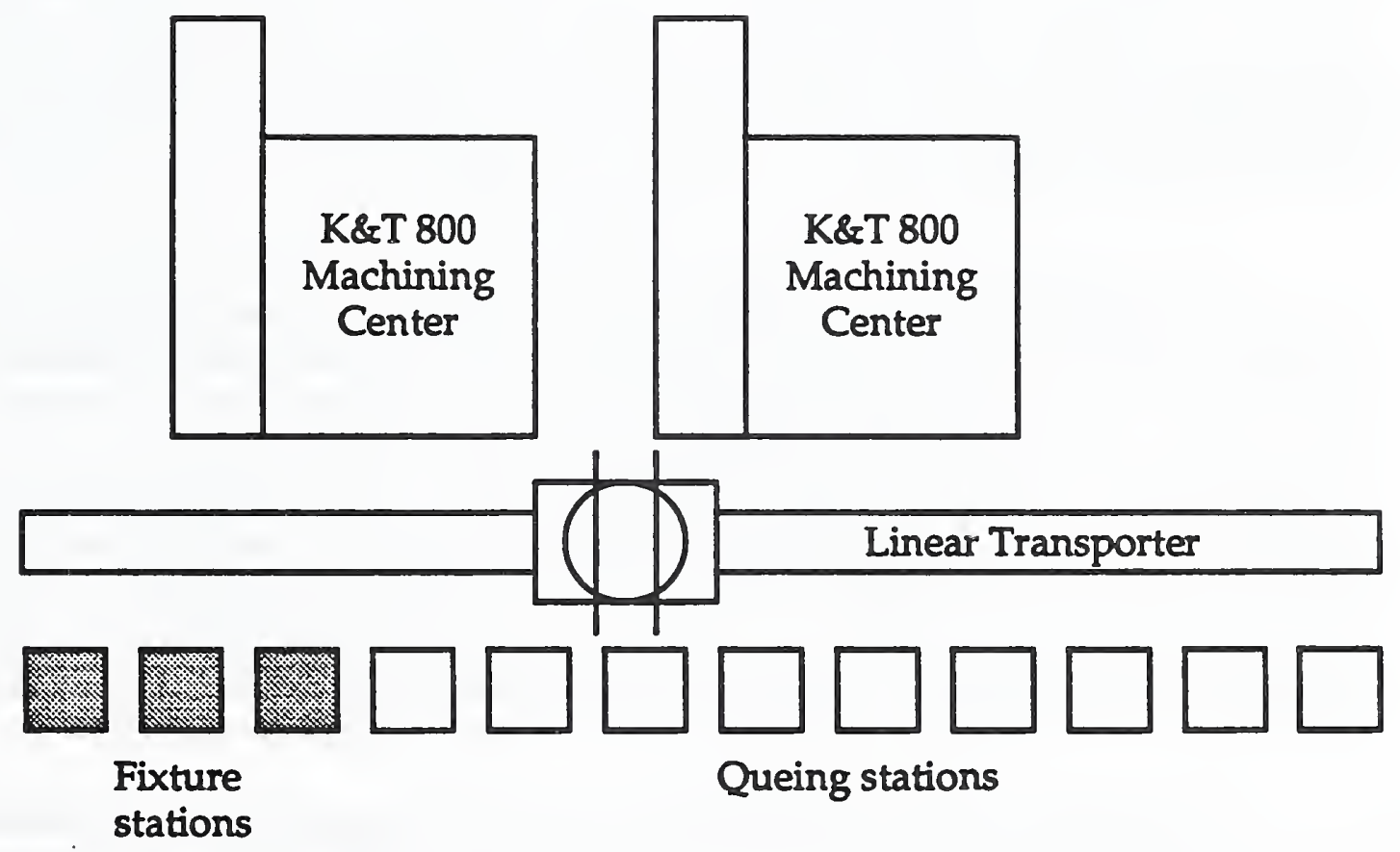

Figure 1-1. The existing flexible machining cell at RIA 
limited basis during the second-shift. If the demand for tool setups is great, second-shift staffing may be increased. The tool rooms maintain notebooks of specifications for standard tool assembly configurations. Tool room staff refer to these notebooks when assembling and presetting tools. Ready assembled tools are grouped by job on shelves in the tool room. Machinists and machine tool operators are responsible for picking up and signing out tooling. Tools are signed out to a job and operator, not to a specific location on the shop floor. Individual tool assemblies are identified only by setup specification number, not by individual serial numbers. Tool tracking is entirely a paperbased system, which does not maintain current location data for tools. With this system, it is very unlikely that tools that are on the floor are reassigned to higher priority jobs.

Operators are responsible for making tool replacement decisions. Assessment of tool wear is made by visually inspecting tools, listening to the tools in cut, monitoring chip formation and part surface finish. If the operator decides that he needs a new tool, he returns the worn tool to the tool room. A replacement tool is assembled by tool room staff on a priority basis while the operator waits. Or, a replacement tool may already been preset for issuance. Very little data is maintained on tool requirements that are common across different jobs. For the most part, no attempt will be made to determine if an identical, unused tool is available for reassignment on the shop floor.

There are two major areas where RIA tool management practices are not typical of industry in general. One of these areas is the adaptive compensation of cutter path for the machining of recoil mechanism chambers on the basis of real-time measurements. The other is the real-time monitoring of cutting parameters. A system has been developed, and placed in use at RIA called Freeze Frame. This system allows the NC programmer or machine operator to capture and record key machining parameters instantaneously, at targeted intervals, during a cut. At the end of the cutting sequence, the captured data is dumped to a printer for analysis by engineers. Cutters for which Freeze Frame data was collected are tagged and set aside for study when they are returned to the tool room. The Freeze Frame effort is leading to a better understanding of the critical factors that affect cutter performance.

Although complete and precise data is unavailable for tooling costs at RIA, it is known to be a significant factor in overall production costs. Tooling inventory is valued in millions of dollars. Other major tool-related cost factors include overhead labor charges for tool room staff, support equipment, tooling-based job delays, etc. Existing tool management practices must be continually refined if tooling costs are to be kept down and production operations are to be made more efficient. 


\subsection{Goals and Expected Benefits}

The goal of this program is to incorporate modern tool management capabilities within the RIA machining cells. The goal of NIST Center for Manufacturing Engineering staff, who are participating in the tool management system (TMS) development effort, is to identify requirements and develop technical specifications which can be used to construct a tool management system with an open architecture. An open architecture would facilitate the implementation of plug-compatible modules which could be upgraded, or replaced, as RIA's needs change in the future. If the Arsenal decides to proceed with development of the tool management system, the technical specifications will provide a basis for selecting commercial products, contracting custom-built systems, and integrating component subsystems. Due to RIA's need for production quality systems, it is expected that all systems will be built and integrated by commercial contractors with proven performance in the field of tool management and/or manufacturing systems integration.

A number of significant benefits are anticipated from this tool management program. Within Rock Island's manufacturing facility, it is expected that there will be:

* increased knowledge of total tooling requirements,

* better understanding of the performance of individual tools,

* accurate on-line tool tracking and inventory data,

* better utilization of tooling, including:

- reallocation of tools on a job priority basis,

- migration of tooling within the facility, and

- fewer work delays due to tooling shortages,

* less possibility to damage to expensive work pieces due to:

- improper tooling,

- tooling with insufficient performance capabilities, and

- worn and broken tools,

* a generally more efficient production system.

An indirect benefit that may result from this effort is the establishment of an open architecture for tool management. This architecture may be used at other DOD facilities, serve as a foundation for standardization, or drive future commercial product development. 


\section{Tool Management System}

\subsection{Objectives}

The primary objective of the Tool Management System (TMS) is to support the tool needs of manufacturing production. Proper tool management should help improve the efficiency of production operations, improve product quality, and minimize the costs. The tool management system should provide a comprehensive and integrated set of functions that all aspects of tooling within an FMS environment. In order to satisfy this objective, the TMS must ensure that:

* tool orders are released to the tool room in a timely fashion,

* tool assembly specifications are clear and unambiguous,

* sufficient quantities of tooling components are always available,

* tools are correctly assembled and preset to proper dimensions,

* all tool movements within the system are positively tracked,

* required tooling is available in tool magazines when needed,

* tools are reassigned to keep active, priority jobs going,

* tools are automatically migrated within cells,

* worn tooling is detected and returned for reconditioning,

* unused tools are returned to vendors for credit or surplused,

* re-usable tools are recycled or reground, as appropriate,

* tool performance is monitored and recorded,

* performance data is factored into future tooling decisions,

* accurate, on-line records are maintained for all tooling data,

* tooling data can be easily accessed by staff and external systems,

* subsystems are not too difficult to operate or maintain, and

* the entire system cannot be crippled by single point failures.

Although the preceding list is by no means exhaustive, it does identify the major important operational characteristics of the proposed RIA Tool Management System.

\subsection{System Overview}

The proposed tool management system is a hybrid system, comprised of both manual and automated components. Furthermore, interfaces must be established between it and other systems which already exist or are on the drawing board at RIA. 
The requirements for the TMS are broken down into seven major functional areas:

* tool management system controller

* tool room

* tool assembly storage and delivery

* tool tracking and data collection

* tool management upgrades to the existing cell

* information services

* CIM system links

The relationship between each of these functional areas is illustrated in Figure 2-1. A brief introduction to each area follows.

Tool management system controller - This controller is responsible for coordinating both automated and manual tool management operations that occur in the tool room and on the shop floor. The system will schedule tool operations, allocate tooling to production jobs, issues dispatches to move tools on the floor, manage the tool-life data, and perform a number of system supervision functions, e.g., initialization, configuration management, data backups, etc.

Tool room - This area, commonly referred to as the "tool crib," is where the inventory of tooling components are stored and tool assemblies are constructed to specification. At presetting stations within the tool room, initial measurements of a tool assembly's critical dimensions are taken and are used by the machine tools to determine offsets. Assemblies are issued from the tool room for use on the shop floor. The tool room staff are responsible for accurately accounting for all issues and receipts. Tool identification readers are located at the presetting stations and in the issue/receipt areas. Tool room staff are responsible for inspecting returned tools to determine whether they should be returned to inventory, reconditioned or scrapped.

Tool assembly storage and delivery - There are both manual and automated options for performing these functions. The automated options include automated guided vehicles and automated storage and retrieval systems for handling tool transport carriers, i.e., racks or trays of assembled tools. Under the automated option, a system controller is required to coordinate operations. The controller processes dispatch orders sent from the tool management system controller. Under the manual option, carriers may be stored on shelves and delivered to the flexible manufacturing system by forklift trucks. 
Tool Management System

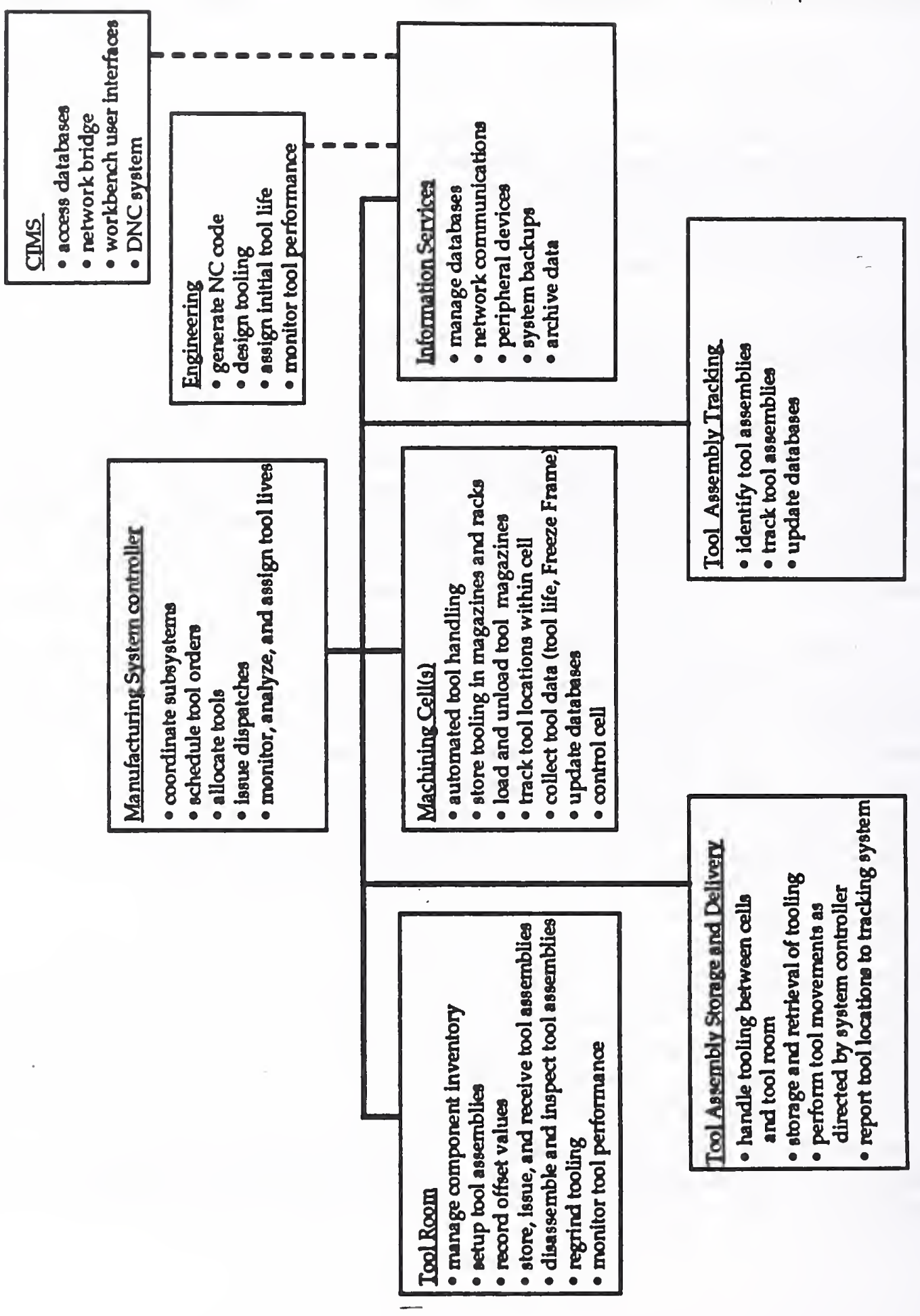

Figure 2-1. Major functional areas of tool management 
Tool tracking and data collection - This system is comprised of readers, sensors and database systems which track the location and monitor the condition of tool assemblies on the shop floor. The system which tracks the location of tool assemblies is based upon readers strategically located at key transfer points in the system. The reader scans an identification chip contained in each tool adapter. The chip contains a serial number code that uniquely identifies the adapter. The tool assembly can then be identified by checking its current configuration data in the database, using theidentification code as a key. Other functions performed by the system include tool wear sensing at the machine tool, tool failure detection during machining, and real-time data collection of cutting parameters (Freeze Frame).

Tool management upgrades to the existing cell - In order to support more sophisticated tool management operations within the existing cell, a robotic tool handling system is required. The overhead robot system carries tool assemblies from a transport carrier pickup and delivery point to other locations within the cell. Tool assemblies may be placed temporarily in tool buffer storage areas or delivered directly to tool exchange points, i.e., ready stations, behind each machine tool. The exchange mechanisms handle the transfer of tools from the ready station into the tool magazine. Each ready station includes a reader to positively identify the tool assembly being transferred. The machine tool controller will update its own magazine log to verify pocket location for retrieval. A read cycle will occur each time a tool is added or removed from a machine tool magazine. Tool-life status and other tool assembly data will be sent to the cell controller when a tool assembly is removed for use in another machine, for rework, or for storage.

Information services - In order for the tool management system to function properly, a number of information systems will be required, i.e., a database management system, a number of tooling and production databases, and a local area network (LAN). Within each work area, a number of computer peripherals will be required, including: terminals, readers, printers, and/or plotters. The information services component of the tool management system is responsible for integrating these systems into a reliable, easy-to-use computing environment.

CIM system links - The TMS will need to share data with a number of RIA systems which are either outside of the FMS environment or above the shop floor. Some of the systems which need to be linked include: the distributed numerical control (DNC) system, other communications networks via bridges or gateways, external database management systems and databases. External procurement systems may need access to inventory and ordering information contained within the TMS.

The requirements for each of these systems are specified in considerable more detail in Sections 3 through 9, respectively. 
Tool Management System

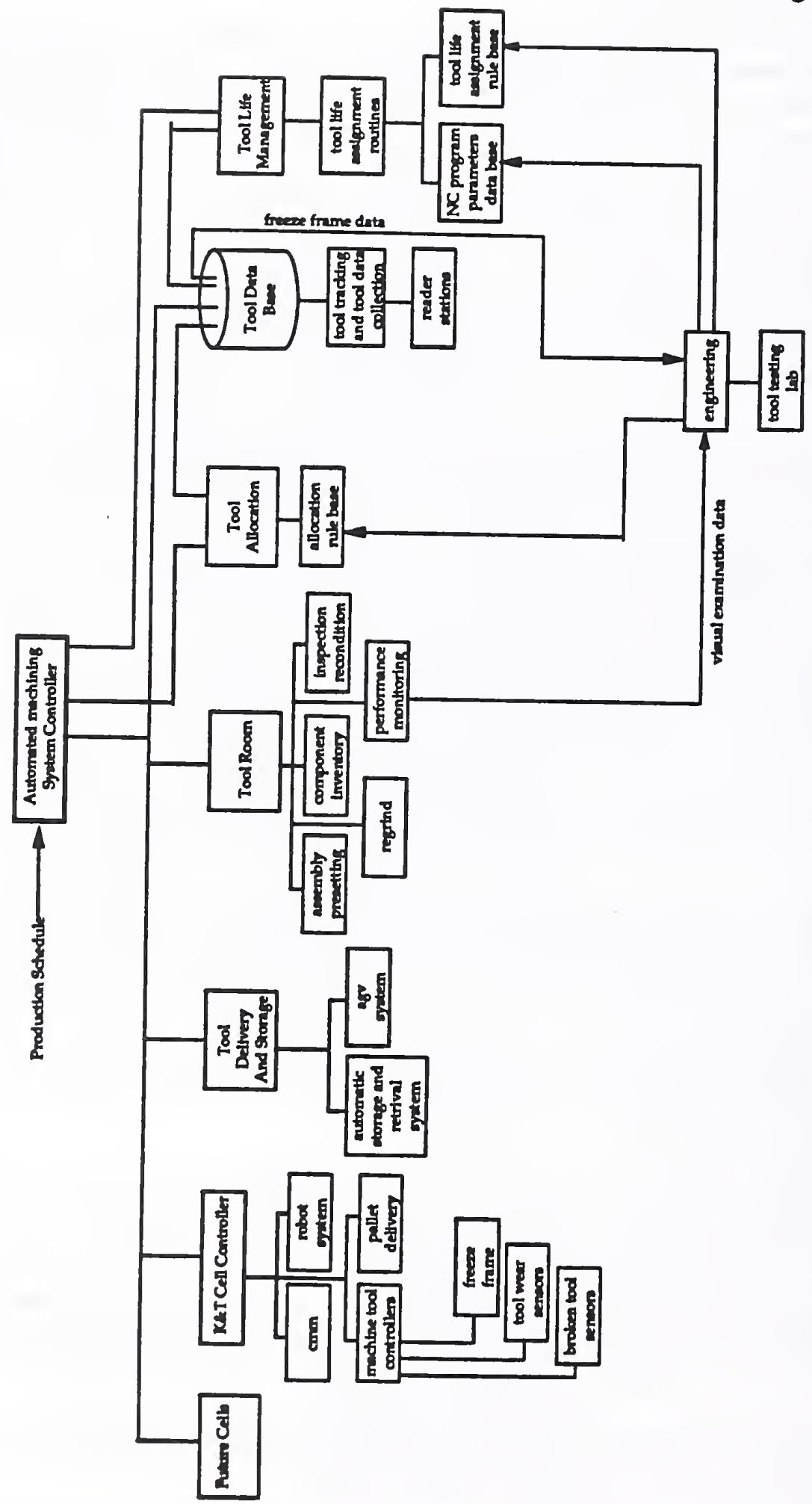

Figure 2-2. Component modules of the proposed tool management system 
Tool Management System

\subsection{Tool Scenario}

At this point, a scenario is an effective way of illustrating how the functions, as outlined above, work together to manage tooling operations and support production operations. This scenario will briefly outline the functions by tracing the movement of a tool and associated data through the system. This scenario is illustrated by Figure 2-2.

Part programming and tool design - The initial requirements for tooling are determined in engineering. Engineering includes tool design, NC programming (i.e., part programming), and process planning functions. Part programmers use part designs to develop NC code, design tooling, and create process plans to manufacture a part. The actual part design drawings are created by designers at another facility. The part programmers at RIA are responsible for designing the individual tool assemblies used to machine the parts. To keep the number of different tool assembly designs to a minimum, programmers are required to select from a set of existing tool assembly designs. If no existing tool setup specification is adequate, a new tool may be designed. The new tool setup specification will then be placed in the database. The tool setup specification contains a bill of materials that identifies the components which make up the tool and a drawing which shows the relationships. The drawing also indicates the critical dimensions of the tool assembly. Next, the job is handed over to a process planner who identifies the sequence of other operations which must be performed on the part, such as heat treatment, painting, anodizing, etc. When planning a job, a process planner checks to see if special tooling is required that is not regularly maintained in inventory in the tool room. If special tools are needed, the tool room staff will enter inventory data for the new items in the database and initiate any required procurements.

Shop order release - Some time after engineering functions are completed, the job will be released to the shop for production by master scheduling. The schedule will specify the jobs that should be accomplished during the next week. The shop manager will enter the information for these jobs at a terminal which interfaces to the tool management system controller. The TMS controller will schedule the jobs for production within the cell and will allocate tools to individual jobs. The status of tools will be updated in the local database to indicate that a tool has been committed to a particular job. About 48 hours before the tool is required on the machine tool, a dispatch order will be sent to the tool room to initiate the tool setup operations.

Tool assembly and presetting - A tool room technician consults a local terminal to determine the next setup job that he/she should work on. The technician has the option of viewing the tool setup specification on the screen or printing out a hard copy on a printer/plotter located in the tool room. Next, the technician draws the required components from inventory drawers and an available tool holder from the rack. The tool is assembled to nominal dimensions, as indicated in the drawing, and is place on the 
Tool Management System

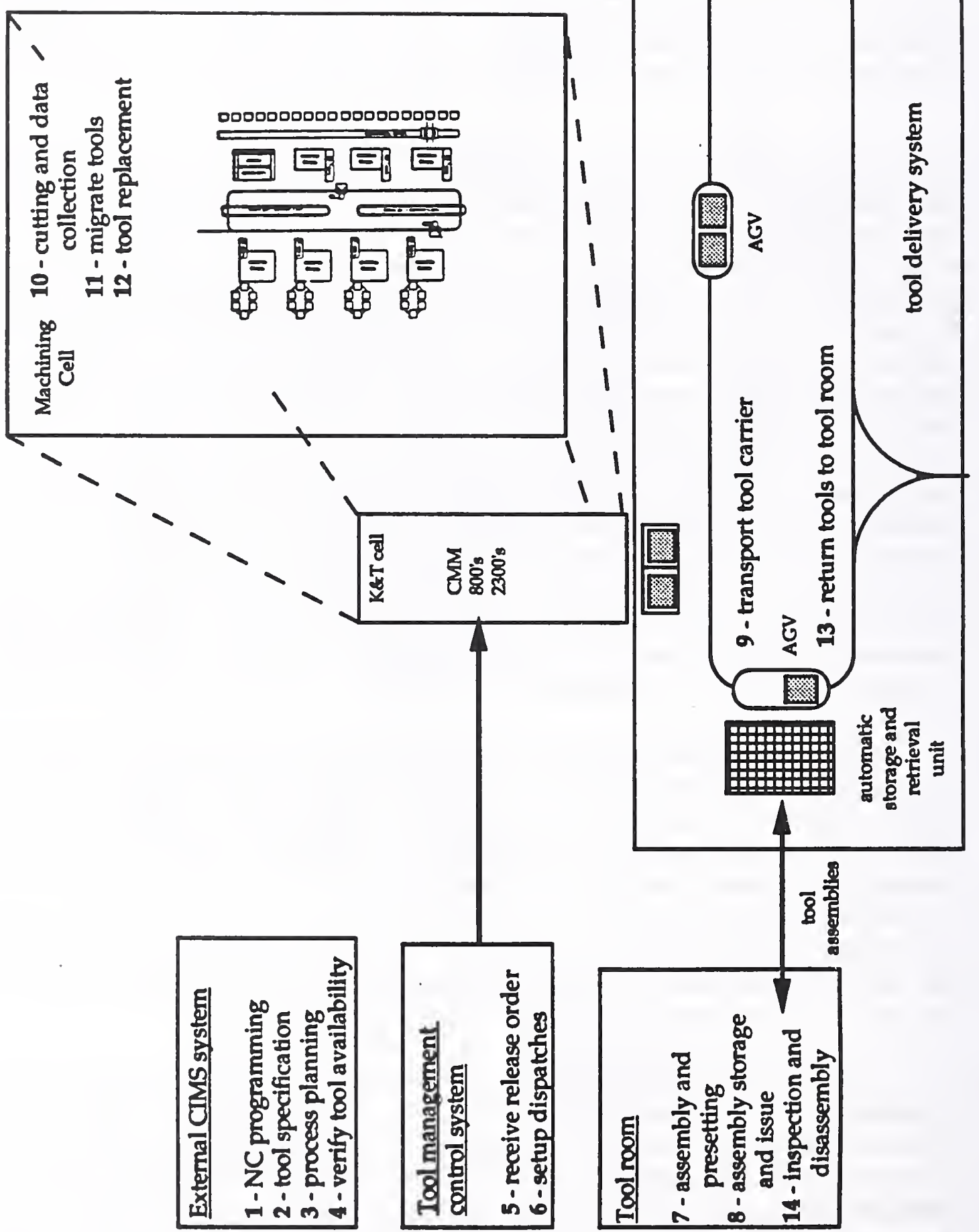

Figure 2-3. An example tool management scenario 
presetting equipment. The technician passes the reader wand over the identification chip to record the identity of the tool holder. Tool offsets are adjust on the basis of dimensional readings made with the presetting equipment. When the technician is satisfied that the dimensions

are within tolerance, he/she enters a keystroke indicating that the tool assembly has been accepted. The tool assembly identification number, tool setup configuration, and critical offsets are then stored in the database. The tool assembly is placed in a storage rack with other tools assigned to the same production job.

Assembly storage and issue - Some time before a tool is needed for production (from a few hours to as much as a day), it must be moved into the cell. The TMS controller sends a dispatch to the tool room ordering that the tool set be issued. Assembled tools are removed from storage racks, wanded with a reader to indicated issuance, and are loaded into tool transport carriers. Whenever the identification code on a tool assembly is checked by a reader, its location data is updated in the TMS database. The carriers may be transported to the cell from the tool room by either automated guided vehicle or forklift truck. If a guided vehicle is used, a dispatch may also be sent to the material handling system which orders the delivery of the next carrier. The transport carrier is placed in a drop-off/pick-up point which is accessed by an overhead robot within the cell. The robot picks tools out of the carrier, moves them past a reader to identify each tool assembly, and moves the tool either to a buffer storage area within the cell or a tool exchange ready station next to a machine tool.

Transfer from cell tool buffer storage - If the tool assembly was temporarily stored in one of the buffer storage areas, it must be moved to the machine tool before the work piece is placed upon the machine for processing. The cell controller commands the robot to pickup the tool in the buffer storage area move it to the exchange point. The tool is delivered to the exchange point and its identification is again checked by a reader. The exchange mechanism (a simple robot arm mounted on the back of the machine tool) loads the tool into the tool magazine.

Cutting operations on machine - When all required tools and the work piece for the next job are present on the machine, it begins execution of the NC program. Tools are cycled from the magazine to the spindle. Before each cut, the tool is touched off on a probe to verify tool length and diameter. At preselected points in the NC program, steps have been inserted to activate the Freeze Frame system. As the controller passes these program points, key machining parameters are stored, e.g., spindle speed, feed rate, spindle torque, axis thrust, date/time, job identification, and feature identification data. When the NC program completes execution, these recorded values are uploaded to the cell controller for transfer to the TMS database. This data is analyzed by engineers to better understand tool performance. This analysis will influence future tool-life estimates and tool selection decisions. 
Migrate tool to highest priority job - A job running on another machine detects that the tool it was using has become worn and has insufficient tool-life remaining for it to be used on the next cut. It advises the cell controller that it needs another tool immediately, i.e., work has stopped. The cell controller searches its database and determines that an identical tool is available on another machine. Through commands to both of the machines and the overhead robot it migrates the tool to the machine with the work stoppage.

Tool replacement - The cell control system must now obtain another tool to replace the tool that was removed from the first machine. It reports to the TMS controller that it has an immediate need for a replacement tool. The TMS controller issues an urgent dispatch to the tool room to order the setup of another tool assembly. Meanwhile the cell overhead robot transfers the worn tool from the machine tool ready station to the tool carrier pickup and delivery point. It loads the tool into an awaiting carrier for return to the tool room. Simultaneously, the tool room staff assembles and presets another tool for immediate delivery to the cell. The tool assembly is delivered to the awaiting magazine and the job can begin, assuming that the work piece is already mounted on the machine.

Return of tools to the tool room - There are three reasons for returning a tool to the tool room: 1) it is worn and unusable, 2) it is not required for jobs in the near future, or 3 ) there is limited storage capacity within the cell. The tool follows the same sequence of operations to return to the tool room. The robot picks it up at the machine tool exchange ready station, wands it by the reader at the pick-up/drop-off point, and loads it into an awaiting tool transport carrier. The forklift or AGV picks up the carrier and returns it to the tool room. Tools are wanded by a reader as they are received back into the tool room.

Inspection and disassembly - Tool room staff wand the returned tool to enter its identification into the computer. The local terminal displays the disposition of the tool. The disposition will indicate either that the assembly is to be either torn down or reworked. If the tool is to be torn down, non-perishable components are returned to inventory, and worn cutters are reground or scrapped. Reworked tools have their cutters replaced, are reset, and returned to a storage rack to await return delivery to the cell. Regrind staff pick up worn tools and recondition the cutting edges.

The preceding discussion illustrates some of the more typical tool management functions. Again, see the functional requirements discussions in Sections 3 through 9 for more detailed information. 


\subsection{Introduction to Subsystem Requirements}

A common template format is used to outline the major requirements for the systems in each functional area or subarea. An example of an area is Section 4, Tool Room. An example of a functional subarea is Section 4.1, Assembly and Presetting. The categories of the requirements template are as follows:

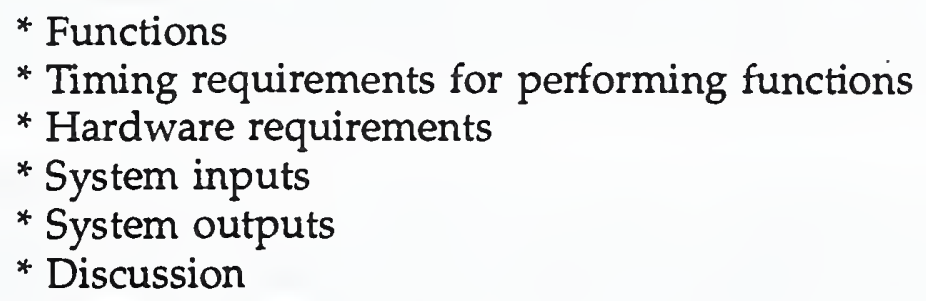

These headings appear in italics in each section or subsection. An attempt is made to identify each requirement once, at either the section or subsection level. For example, if one computer system is needed to satisfy the functions in several subsections, it will be identified at the section level. Descriptive text has been made as brief and as concise as possible.

Under the "System inputs" and "System outputs" heading, a single letter codes are used to indicate the devices or systems which send or receive that data.

(D) - database or database management system

(E) - physical equipment

(P) - printer or plotter

(R) - tool identification reader

(T) - computer terminal, display monitor, or teach pendant

(S) - supervisory or control system 


\section{Tool Management System Controller}

The Tool Management System (TMS) controller is responsible for coordinating tool operations among several shop floor areas. The major areas that the system must directly or indirectly coordinate include the tool room, the tool storage and delivery system, and the machining cells. It is responsible for allocating tools to individual production jobs, scheduling tool room setup activities, ordering the delivery of tool trays to the production cells by the material handling system, and performing tool-life monitoring, analysis, and assignment functions.

The system is comprised of computer hardware, software, peripherals and interfaces to other parts of the tool management system. The controller will also have supervisory functions which pertain to the software nature of the system, e.g., providing operator consoles and user interfaces, initializing systems, managing backups of data and recovery from errors, and coordinating degraded modes of operation.

\subsection{Tool Scheduling and Allocation}

The Tool Management System schedules the setup of tool assemblies in the tool room, the delivery of tooling trays by the automated guided vehicle system, the transfer of tools within the cell, the disassembly, and/or the reconditioning operations. Once delivery schedules are established, the Tool Management System determines the physical allocations of tools to machines in accordance to cell loading instructions. Tool assemblies are allocated to specific production jobs in the system. Allocations are released and reassigned to ensure that tooling is available for the highest priority jobs.

\section{Functions}

* The TMS will contain software which generates schedules that are used to determine when commands will be dispatched to the various subsystems. Activities that need to be scheduled include tool assembly and presetting, storage and delivery of tools to the machining cell, and transfers between machines. The TMS is also responsible for determining the physical allocation of tools to jobs and machines. It does so by interacting with a machine loading function responsible for allocating jobs to machines. Tool allocation is driven by the job schedule. The TMS provides user interface software. Operators should be able to:

- View the established schedules.

- View the commands that have been initiated and that are currently executing. 
- Monitor the progress of the subsystems in carrying out tool allocations commands.

* The tool allocation system must provide feedback to the scheduling software when tool allocation shortages or conflicts will require changes in the production schedule. Discussions of the tool scheduling and allocation procedures follow in the next section.

Timing requirements for performing functions - NA

\section{Hardware requirements}

* One data entry and display terminals shall be provided local to the control room. The display shall have sufficient graphic resolution to display schedules as Gantt charts or network displays.

* The user interface provided on this terminal shall be support data entry and display operations for at least the following types of activities:

- View the current (daily/hourly/...) Job Schedule.

- View the corresponding (daily/hourly/...) Tool Assembly and/or Delivery Schedule.

- Override the tool allocation plan, and provide for manual reassignment.

- View the current Tool Allocation Plan for a specific Work Order.

- View the current Tool Allocation Plan by Tool Type.

- View the current Tool Allocation Plan by Tool Identification Number.

- View the Resource Requirements List for a specific Work Order.

- Determine the location of any Tool Type via a Tool Location Report.

- Determine all the tools at a particular location via a. Inventory Location Report.

- Initiate the printing of hard copy reports

* The user interface shall employ a password security system which prevents unauthorized personnel from modifying the Tool Allocation Plan.

* A system shall be provided by which trained RIA computer personnel can modify tool allocation algorithms without contractor assistance. Documentation shall be provided which clearly illustrates the method by which allocation algorithms, may be modified. Documentation shall provide at least two examples which illustrate allocation on the basis of: 
1) job priority, and 2) most readily or quickly available tool that is not scheduled for use within a specified period of time.

System inputs

* Operator display selections (T)

* Operator hard copy report requests (T)

* Job schedules $(D, T)$

* Job schedule changes $(\mathrm{T})$

\section{System outputs}

\section{Discussion}

* Tool allocation schedules $(T, D, S)$

The daily production plan is used by the tool scheduling and allocation system to determine tools assignments and deliveries. The TMS develops its internal tool scheduling plan that aims to satisfy the production requirements. A time-phased schedule of the tooling requirements is generated; it is then augmented with the machine loading assignments of each job to each machine.

To execute the job schedule, the TMS identifies the location of existing tooling and the shortages of required tool assemblies. It generates the necessary requests to accomplish the required tool deliveries. It also interfaces to the machine loading system which identifies the assigned locations of production jobs. Based on these locations, it places tools transfers requests.

The job schedule specifies when and where each job should run. The resource requirements list identifies the required tools and the tool-life required for each job. The database maintains the location and the available life remaining on each tool assembly. The cell tool allocation software must consider job priorities, tooling availability, and remaining tool-life in order to develop the tool allocation plan which identifies each tool assembly that has been assigned to a specific job number. The software must release and reallocate tool assemblies automatically as required to satisfy schedule revisions and job priority changes.

A job schedule may be generated in any of several alternate ways: 1) externally by a higher level system that is responsible for the supervision of the TMS and the machining cell(s), 2) externally by the machining cell, 3) manually by shop management and/or system operators, and 4) internally by the tool cell controller. 

schedules:

The TMS software will use the following types of information to generate tools

* the jobs released for production,

* the detailed tasks which are required to carry out each job,

* task duration data,

* the status of automated subsystems,

* the availability of tool room staff and required component inventory,

* inventory shortages or allocation conflicts reported by the tool allocation software.

The task duration data are determined from standard operation times maintained as reference data in the data base or calculated as expected durations on the basis of task specific data. The software will maintain a current copy of the schedule in the common data base.

User interface software for the scheduling system should support several different display screens. A Gantt chart display shall be available which illustrates the sequence of tasks that are queued at each resource. For each task start and stop times should be displayed. Slack periods for each resource should also be indicated. A network display shall be available which illustrates the precedence relationship between tasks for any job.

Control room staff will need to review periodically the tool allocation plan that is generated by the cell controller. Operators may occasionally feel the need to override decisions made automatically by the tool allocation software. The user interface must be designed to give system operators this flexibility. The interface should also permit operators to indicate when the system may override or release an allocation made manually. For example, if the job is completed or cancelled, corresponding tool allocations should be released. Also, operators may want to establish a resident set of tools at a particular machine. In this case, the system could assign and release tool allocations among different jobs as long as the tools did not leave a particular machine. Other more complicated variations of resident tool and tool migration scenarios require that RIA staff are provided with a mechanism for modifying tool allocation algorithms.

The tool allocation software must manage not only tool holders and tool assemblies, but also all components in inventory in the tool room. Reorders of component stock can be initiated when allocation records indicate a projected deficiency of a required inventory item. If a critical shortfall in component stock does occur, terminal display screens should provide operators with a mechanism for identifying components in assembled tooling. The operator should be able to determine at the terminal which other tool assemblies use the same component. 


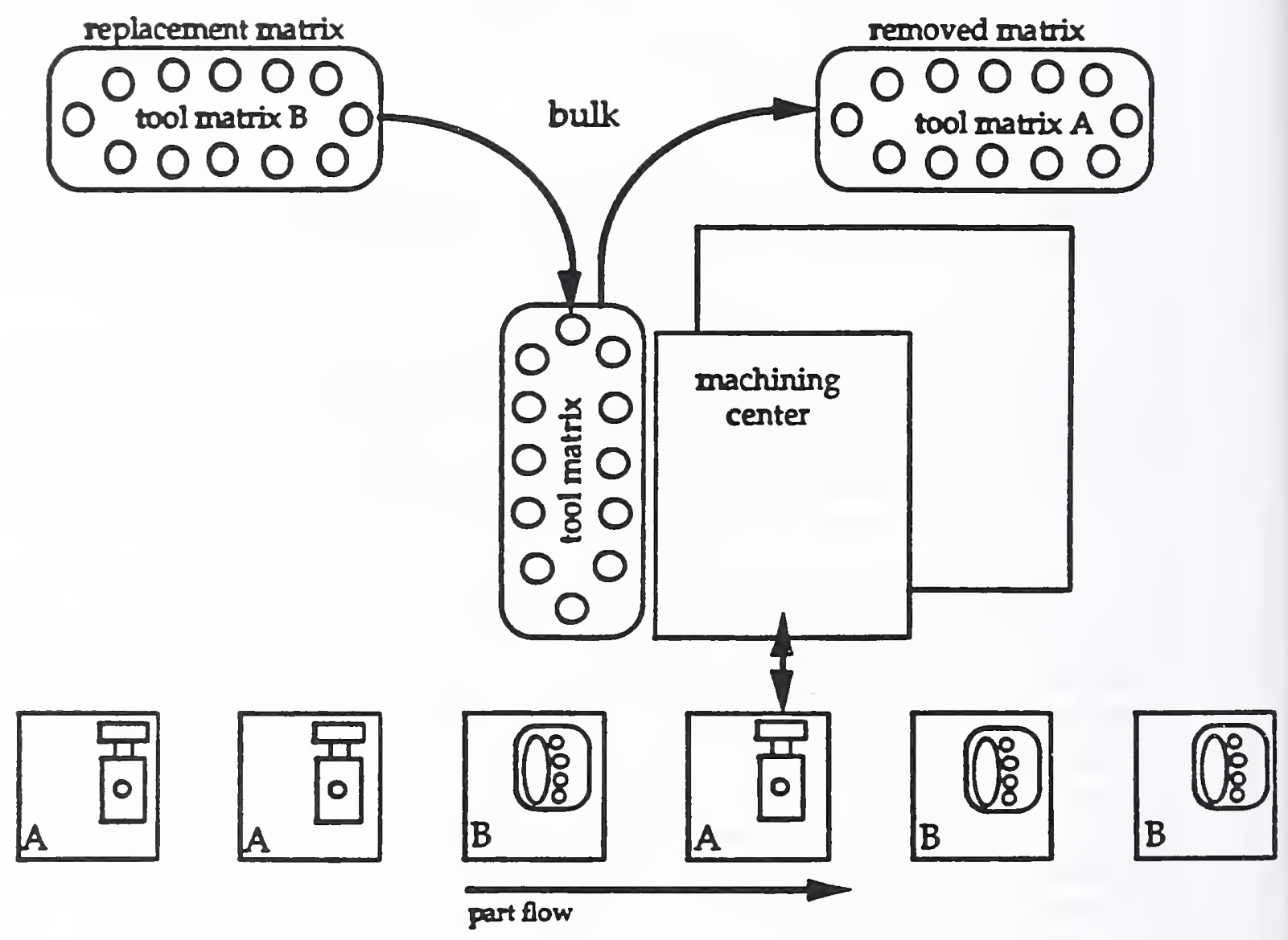

Figure 3-1. Allocation strategy: bulk exchange 


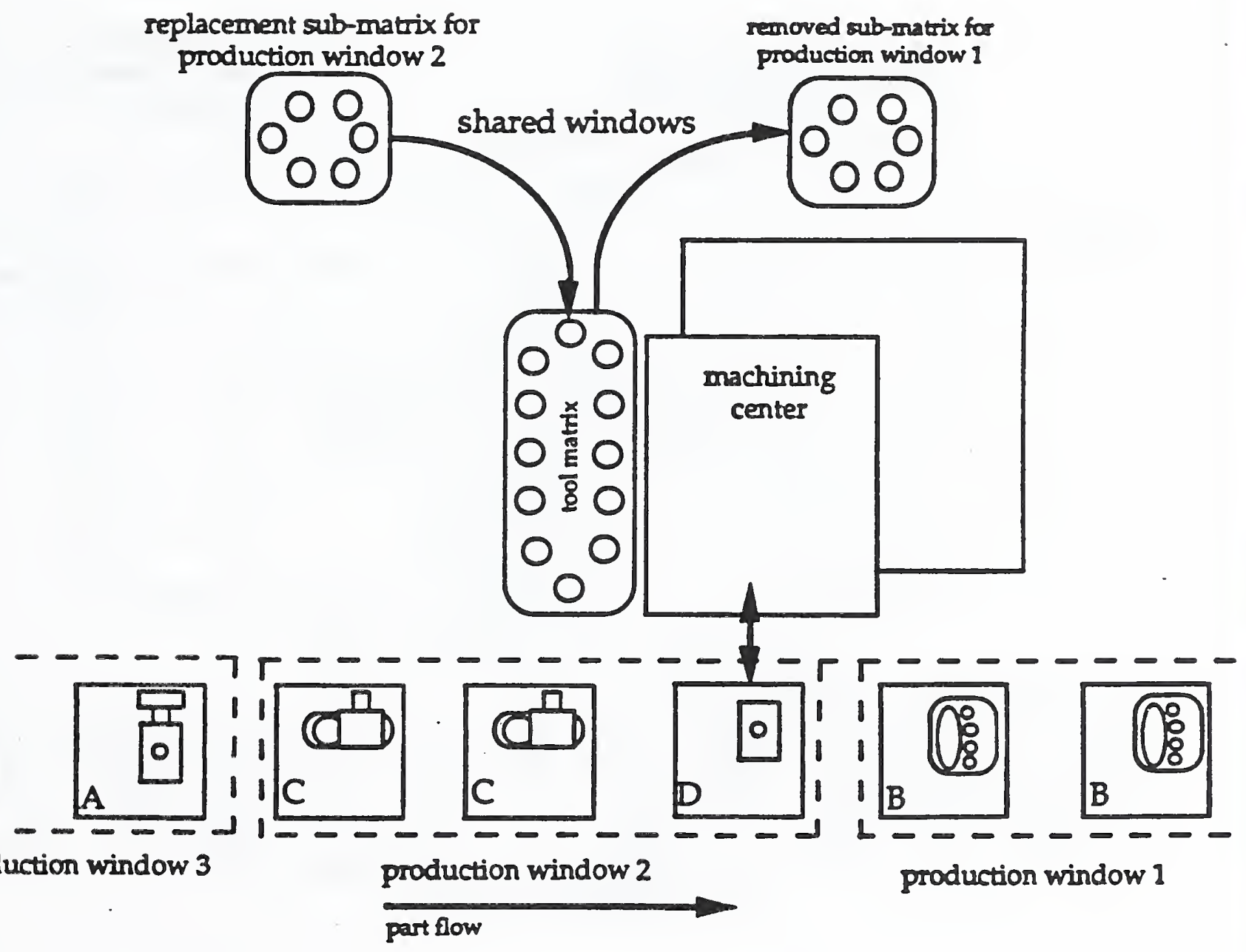

Figure 3-2. Allocation strategy: shared tooling 
Tool Management System Controller

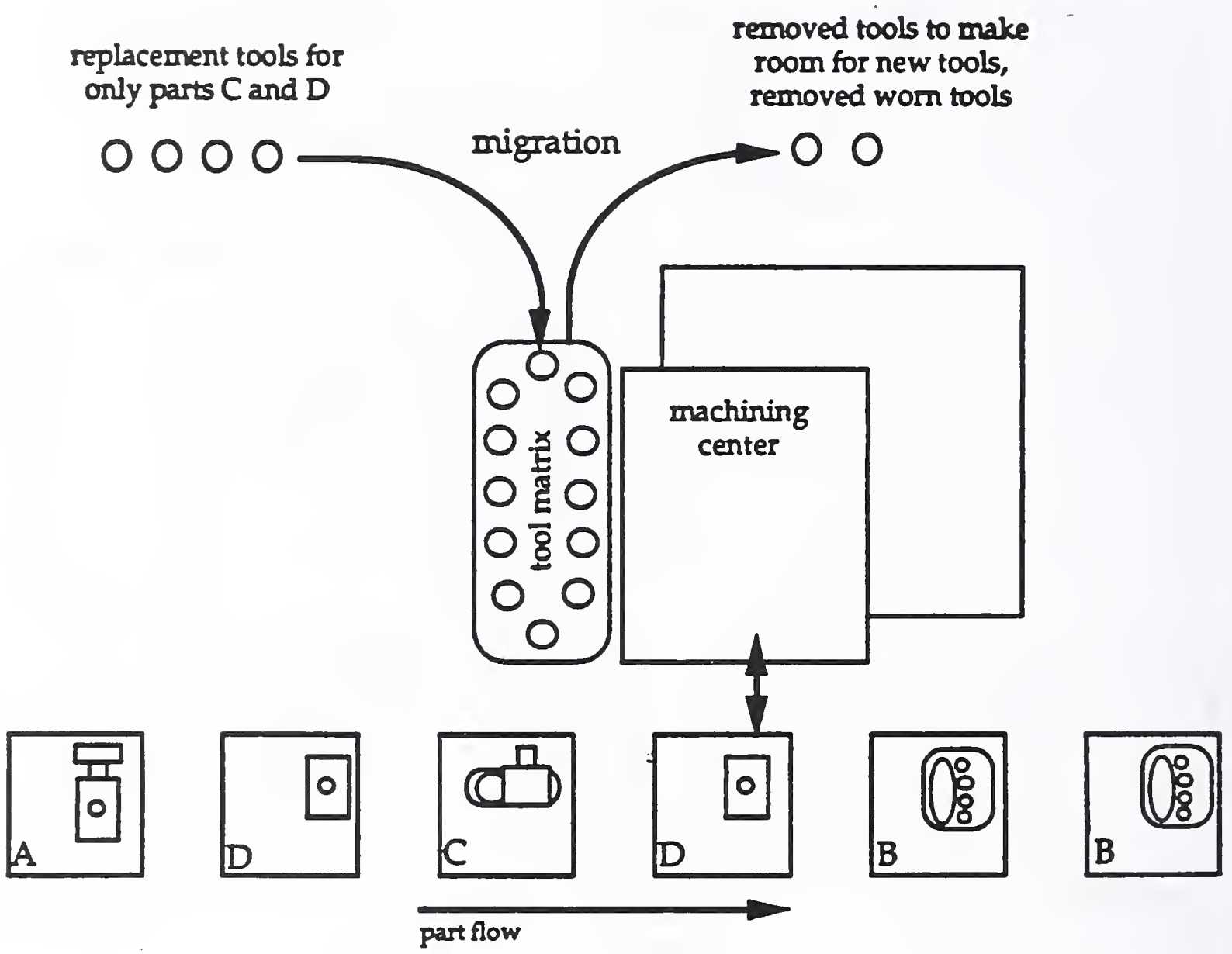

Figure 3-3. Allocation strategy: migration 


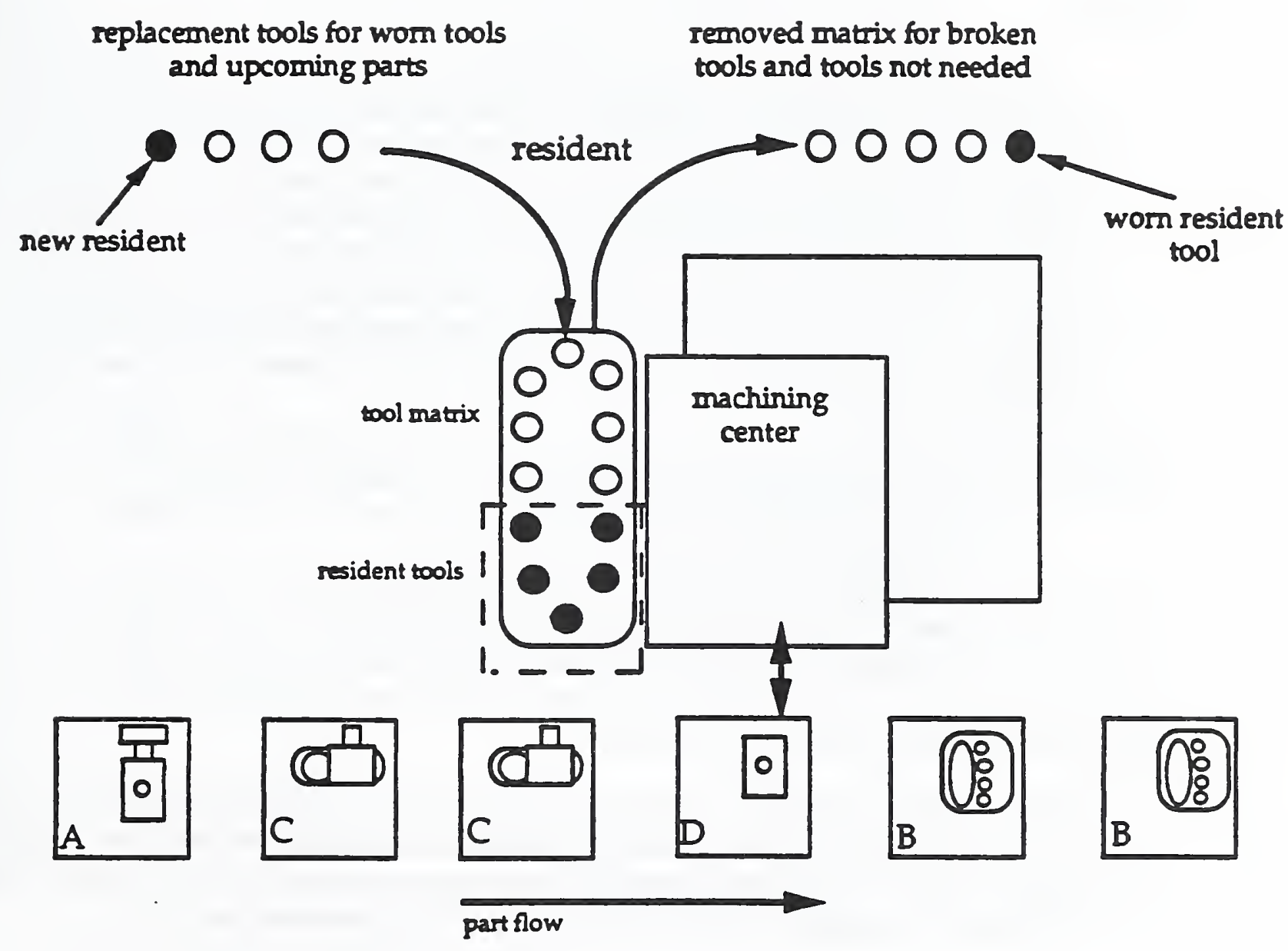

Figure 3-4. Allocation strategy: resident tooling 


\subsection{Tool Dispatching}

Dispatches are issued to the automated systems (e.g., the material handling systems, the ASRS, etc.) to initiate tool movements, according to tool allocation decisions. The Tool Management System is responsible for monitoring the proper execution of dispatching orders, synchronizing tool deliveries, initiating tool deliveries within cells and from storage, updating system status.

\section{Functions}

* The TMS Tool Dispatching function is responsible for tracking the development of the tool scheduling and allocation plan developed by the Scheduling function of the TMS. It synchronizes and coordinates the activities of the tool delivery system between storage, tool room, and work stations. It provides data terminals and user interface software to display dispatching status. The user interfaces also support manual intervention to override tool allocation decisions made automatically by the system. The system provides support information for tool room operators in order to accomplish the manual overrides. This should include at least the following reports:

- Machine utilization loads - current and future.

- Jobs allocations per machine.

- Jobs status and remaining slack per job.

- Current tool allocations per work station.

- Tool room status of assemblies and backlog.

- Priority allocations for tool setup requests.

Timing requirements for performing functions

* Planning data updates - The Tool Allocation Plan should be current at all times. For example, the plan should be updated whenever the job schedule changes, a job completes, a tool replacement becomes necessary, a tool will not be available as planned, and the operational status of a machine changes.

\section{Hardware requirements}

* One data entry and display terminal shall be provided local to the control room, which displays tool assembly locations, tool assembly setup queue, job queues, tool allocation decisions, etc. 
* The user interface provided on this terminal shall support data entry and display operations for at least the following types of activities:

- View the current Dispatch List for each subsystem.

- Override automatic dispatch instructions generated by the system.

- Provide necessary support information in order to accomplish the manual override. This should at least include some of the following information: current and expected machine utilization, tool delivery system status, tool room performance, job completion status and slack per job.

- Alert the tool room staff with either an audio or visual alarm when their attention is required to supervise the tool dispatching processes. This function is especially needed when the tool delivery system cannot satisfy the dispatch list requirements on time.

- Initiate the printing of hard copy reports

* Hard copy reports that shall be printable on the control room printer shall include the current Tool Dispatch List, and the various reports necessary for manual override of the tool dispatching instructions.

* The user interface shall be easy to use. Control room personnel should be able to become qualified in its operation with one day of hands-on training. The user interface shall employ a password security mechanism which prevents unauthorized personnel from modifying the Tool Dispatch List.

* Documentation shall be provided which clearly illustrates the method by which dispatching procedures may be modified. Documentation shall provide at least two examples which illustrate dispatching on the basis of: 1) tool allocation plan, 2) job priority, and 3) backlogs at either the tool room or the tool delivery system.

System inputs

* Operator display selections (T)

* Operator hard copy report requests (T)

* Modifications to tool allocation plans (T) 


\section{System outputs}

* Tool allocation plans (T,D,S,P)

* Job schedule (T,D,S,P)

* Location inventory reports $(\mathrm{T}, \mathrm{D}, \mathrm{S}, \mathrm{P})$

* Tool location report (T,D,S,P)

* Allocation status feedback to the scheduling functions $(T, D, S)$

\section{Discussion}

Dispatching refers to using the tool allocation plan to generate a list of instructions directed to either the tool delivery system or to tool room operators (mostly in the case where the tool delivery system is failing to meet the tool allocation plan). Commands are generated and are issued to the appropriate subordinate components of the tool delivery system (robot, AGV, ASRS) as messages transmitted over the network. A command may contain several orders. Each order has an identifying number, a version or update number, a task which is specified as a parameterized work element, and an action to be taken. The command data is updated and a new message will be passed to the appropriate subsystem any time a new order is added to the current dispatch list. If the required action on any order in the dispatch list is revised, an updated command will be issued to the subsystem.

\subsection{Tool-life Monitoring, Analysis, and Assignment}

The controller must manage the tool-life data that is used to determine gross tooling requirements and make tool replacement decisions. Sources for this data include the inspection and performance monitoring activities within the tool room, machining centers on the shop floor that are equipped with Freeze Frame, and tool wear/breakage sensors. The controller must analyze incoming data and continually update tool-life assignments.

\section{Functions}

* The TMS will maintain tool-life records that are stored in the data base. The system must provide user interfaces for setting initial tool-life expectancies by RIA engineering staff. Monitoring functions will be responsible for accumulating actual tool use data from the machining cell. An analysis function will interpret data obtained by the monitoring system. It will determine whether or not tool-life is being consumed in accordance with expectations. 
The assignment functions will be responsible for:

1) setting the initial expected tool-life values associated with new tools,

2) calculating the effective life remaining on a tool at the completion of each cutting situation, and

3) updating the expected tool-life remaining after each tool use.

Timing requirements for performing functions

* Tool-life data assignment - The Tool-life Records should be current at all times. Frequency of updates will be dependent on the capabilities of the cell control systems. For example, until on-line tool health (wear condition) monitoring is possible, it is likely that the cell control system may only update tool-life data at the end of an NC program. The preferred approach would require that an NC program is only authorized to start if the remaining tool-life of all tools needed is judged to be sufficient for the completion of the part program. Tool-life will then be updated at the end of the NC program. By keeping tool-life data current at all times, a tool may be reallocated and migrated to another job on another machine any time that the tool is not currently in the spindle - provided the tool allocation plan authorizes it.

\section{Hardware requirements}

* One data entry and display terminals shall be provided local to the control room which display tool-life monitoring, analysis and assignment data. The user interface software shall permit RIA staff to manually intervene to adjust tool-life assignments that have been set automatically by the system.

* The user interface provided on this terminal shall support data entry and display operations for at least the following types of activities:

- View the Tool-life record for any tool number

- Initiate the printing of hard copy reports

* Hard copy reports that shall be printable on the control room printer shall include the current Tool Allocation Plan and various inventory reports. 
* Documentation shall be provided which clearly illustrates the method by which life analysis algorithms and assignment routines may be modified.

System inputs

* Operator display requests $(\mathrm{T})$

* Operator hard copy report requests (T)

* Modifications to Tool-life Data (T,D)

System outputs

* Tool-life reports $(T, D, S, P)$

* Recalculated remaining tool-life $(\mathrm{T}, \mathrm{D}, \mathrm{S}, \mathrm{P})$

\section{Discussion}

The primary function within the TMS that needs tool-life data is tool allocation. The tool allocation software will require tool-life data in order to determine the quantity of tools that will be required for machining each part. Ideally, the tool allocation software will guarantee that each tool dispatched for a job has enough useful life to complete the cutting cycle. For tools whose useful life exceeds one part, the software will have to calculate the number of parts that can be machined before the tool will require reconditioning.

It is likely that the machining centers will only be able to track tool-life in terms of minutes of cutting time used. Cutting time will be recorded in two ways: 1) as accumulated cutting time or 2) as cutting time remaining until tool-life is expended. In either case, a machining center will record the consumption of tool-life at a uniform rate, i.e., one minute of active spindle time means one minute of life used. Unfortunately, this simplistic approach does not consider that under different cutting conditions a tool's life may be consumed at different rates. The tool-life software will have the responsibility of adjusting for and revising tool-life based upon these different rates. This may be done by either normalizing useful tool lives with respect to cutting parameters, or simply by compiling all the knowledge available regarding tool usage per job.

For each tool assembly, the software will maintain a tool-life record in the data base. The tool-life record will contain two types of data, predicted tool-life and remaining tool-life. When a consumable tool component is drawn from inventory it is assumed to have $100 \%$ of its predicted tool-life remaining (i.e., when the tool is new or reground; predicted tool-life = remaining tool-life). The tool-life will be represented in terms of the expected number of minutes of cutting time available under a standard set of cutting conditions, i.e., a particular material hardness with appropriate speed/feed 
rates or volumetric material removal rate. This will be true whether the tool is new or has been reconditioned. The tool-life assignment software will be responsible for calculating the increase or decrease in available cutting time for the tool under the next set of cutting conditions. The remaining tool-life field within the tool-life record will be set to this adjusted value. This value will be provided to the machining cell for loading into its cutting time data registers.

After the tool is used, the machining cell will provide the number of minutes of life used to the tool-life software which will calculate the normalized tool-life used under the cutting conditions most recently experienced by the tool. The tool-life assignment software will also calculate an adjustment and record a new remaining tool-life value in the data base. Data obtained from tool wear or breakage sensors may be used to override timer values. Should additional tool wear monitoring methods be incorporated (chatter/vibration measurement, spindle torque/deflection, degradation of surface finish), the tool-life assignment software will then modify the remaining tool-life accordingly. Of course, if tool breakage is detected, the remaining life of the tool assembly should be set to zero. It should also be marked for rework. The analysis function will be responsible for determining which tools have an insufficient tool-life remaining for its Tool Usage Profile. If the life is insufficient, it will be flagged in the data base for reconditioning.

The tool usage profile will identify a set of minimum remaining tool-life requirements for each tool and job type. It will also identify a recondition point which is describe in terms of minutes of tool-life remaining. A tool will automatically be marked for reconditioning when its remaining life drops below this point. Reconditioning requirements should be determined by considering the job schedule, the tool allocation plan, the tool usage profile, and tool-life data. 


\section{Tool Room}

The tool room is where the inventory of basic tool components is maintained, e.g., tool holders, extenders, drills, mills, inserts, etc. Functions performed within tool room include: inventory management, tool assembly, tool data recording, presetting, kitting, issue and receipt of tools, disassembly, inspection, performance monitoring, reconditioning and regrinding. The TMS is responsible for providing information which is used to coordinate activities within the tool room. See Figures 4-1 and 4-2.

\subsection{Assembly and Presetting}

Tool assemblies are constructed from basic components (e.g., tool holders, adapters, extenders, drills, mills, inserts, etc.) which are drawn from inventory cabinets. Existing tool assemblies are reconditioned by replacing worn consumable components (e.g., drills, mills, inserts, etc). Newly constructed tool assemblies and reconditioned tool assemblies are set to nominal dimensions as specified in tool setup diagrams. Tool dimensions are measured and actual values are electronically recorded in the tool data base.

The TMS will provide data terminals and user interface software to coordinate operations at an existing assembly and presetting area within the tool room (see Figure 4-3). The TMS will use these terminals both to provide data to tool room personnel and to acquire data from the staff. The TMS must also provide interfaces for data acquisition to existing presetting stations and a planned tool identification system.

\section{Functions}

* Set-up tool assemblies for production operations

* Read tool identification on tool holder

* Transmit tool assembly data (e.g., offsets) to tooling data base

* Update tool assembly data base when:

- tool assemblies are setup

- tool assemblies are reconditioned

- completed tool assemblies are stored in the tool room

* Update tool component inventory when components are removed 


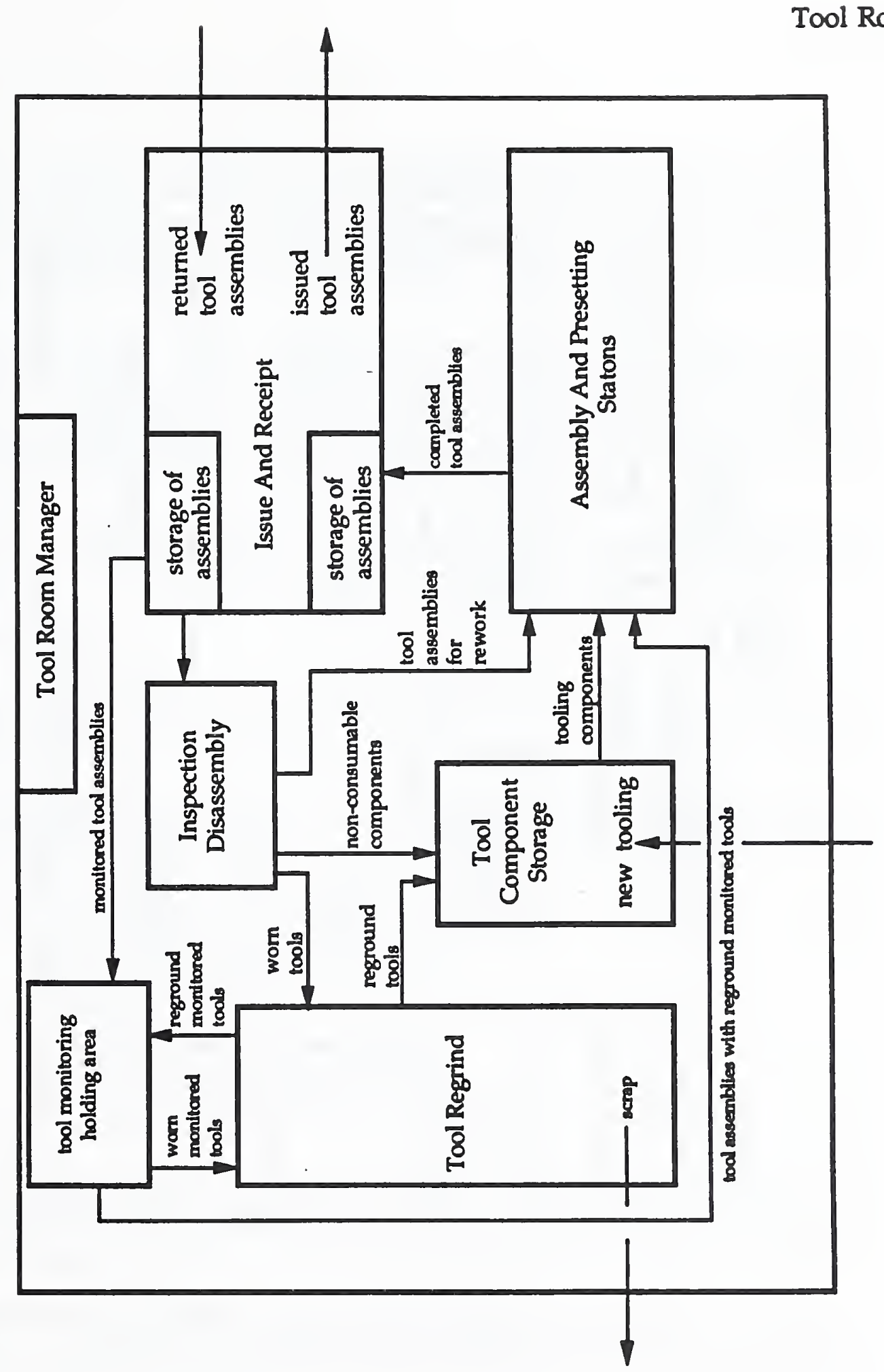

Figure 4-1. Tool component and assembly flow within the tool room 


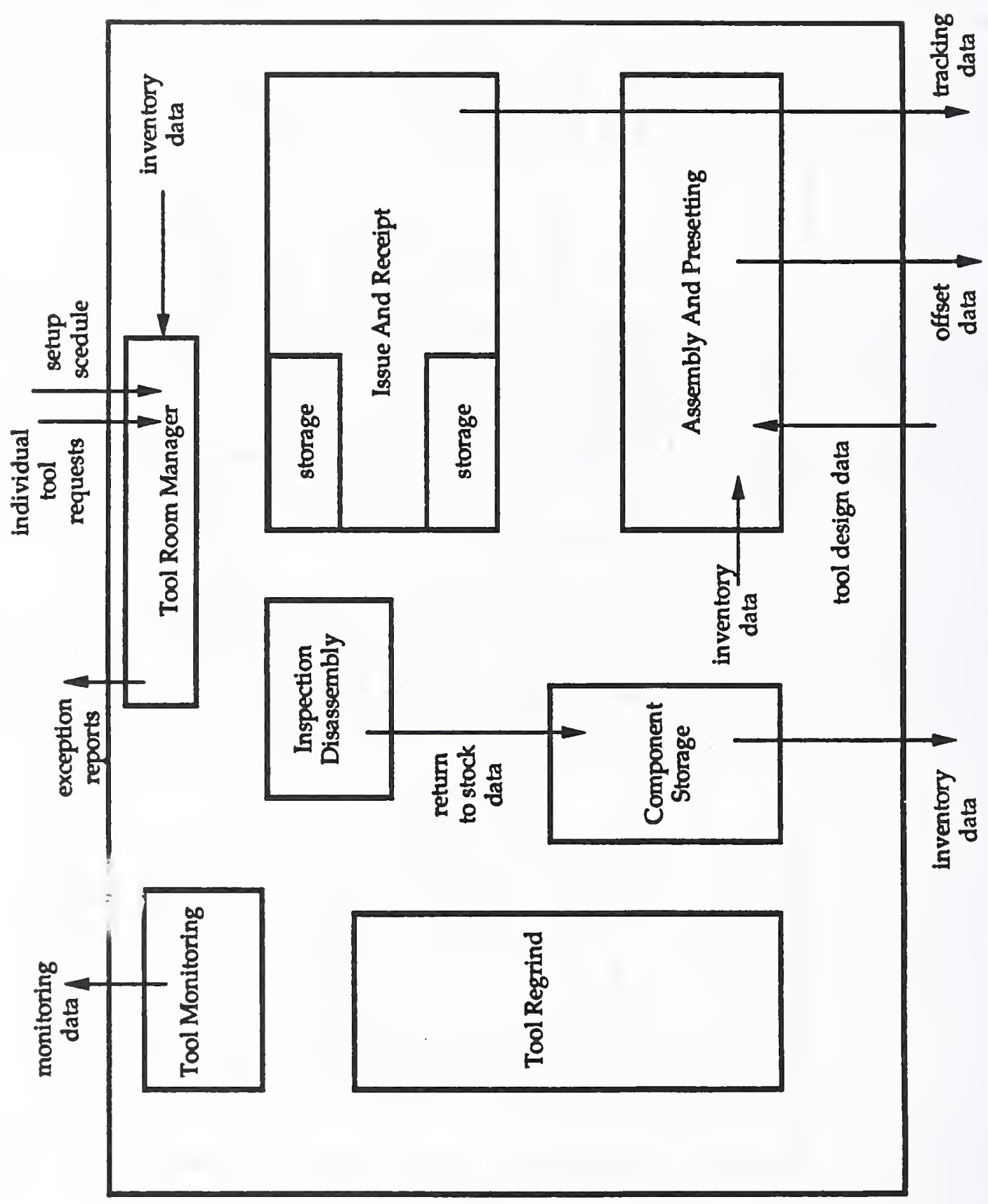

Figure 4-2. Data flow within the tool room 
Timing requirements for performing functions

* Tool identification data - Data acquisition from the tool identification system (micro-chip reader stations) should be completed within 3 seconds after operator indicates that tool identification data is ready for acquisition. The system should be immediately ready to respond to the next operator request after completing data acquisition.

* Presetter data - Data acquisition from the presetting equipment and transmission to the tool data base should be completed within 10 seconds after an operator indicates that measurement data is ready for acquisition by the TMS. The system should be immediately ready to respond to the next operator request after completing data acquisition.

* Tool data base transactions - Data base transactions should be completed within 10 seconds of an operator initiating the transaction.

\section{Hardware requirements}

* One terminal shall be provided for each tool assembly and presetting station. See Section 8.4 for terminal requirements.

* The user interface provided on this terminal shall be support data entry and display operations for at least the following types of activities:

- Check the current tool setup schedule

- View a tool setup specification

- Display a tool assembly drawing

- Determine the availability and location of components in inventory.

- Report the status of a tool setup

- Generate tool room exception reports

- Initiate the printing of hard copy reports

* One printer shall be provided for shared access by all assembly and presetting terminals within the tool room area. See Section 8.4 for printer requirements.

* One tool identification reader shall be provided at each tool setting station. Hardware interfaces shall be provided for the purpose of communicating acquired data from the tool presetting equipment and the tool identification system. 
System inputs

* Tool setup schedule (D,S,T)

* Tool setup specification (D)

* Tool assembly drawing (D)

* Tool availability report (D)

* Data acquisition status indication (R)

* Tool identification (R)

System outputs

* Completed tool assemblies (E)

* Tool offset data acquired from presetting equipment (D,E,T)

* Tool assembly data (D,T)

* Operator display selections (T)

* Operator hard copy reports (P)

* Tool room exception reports $(P, T, D)$

* Tool setup status $(T, D, P)$

\section{Discussion}

Tool room staff will need to be provided with a tool setup schedule which identifies the quantity of each tool number that must be setup to satisfy current job requirements. For each tool number, the tool room staff requires a tool setup specification, which includes an assembly drawing, a list of components, and quantities required. The assembly drawing illustrates the relationships between each component used in the tool assembly.

Presetting is performed on tool setting equipment which records exact tool dimensions or offsets for the tool assembly and stores them in the data base by tool identification number. The tool assembly data, i.e., tool setup specification, is comprised of a tool assembly components list and tool assembly drawing, which is stored by tool number. Each instance of an assembled tool number is associated with a unique identifying number, the tool identification number, i.e., the micro-chip number. The record of tool dimensions, the tool offset record is stored by tool Identification number. This record is read from the data base and sent to a machine tool controller when the tool assembly is loaded into that machine tool's magazine.

Tool room staff must report to the system the completion of assembly and presetting for each tool setup. They must also report exception conditions that might affect schedules, i.e., tool room exception reports. Exceptions that can be reported by tool room staff must include: unavailability of components, tools, equipment, and staff. 


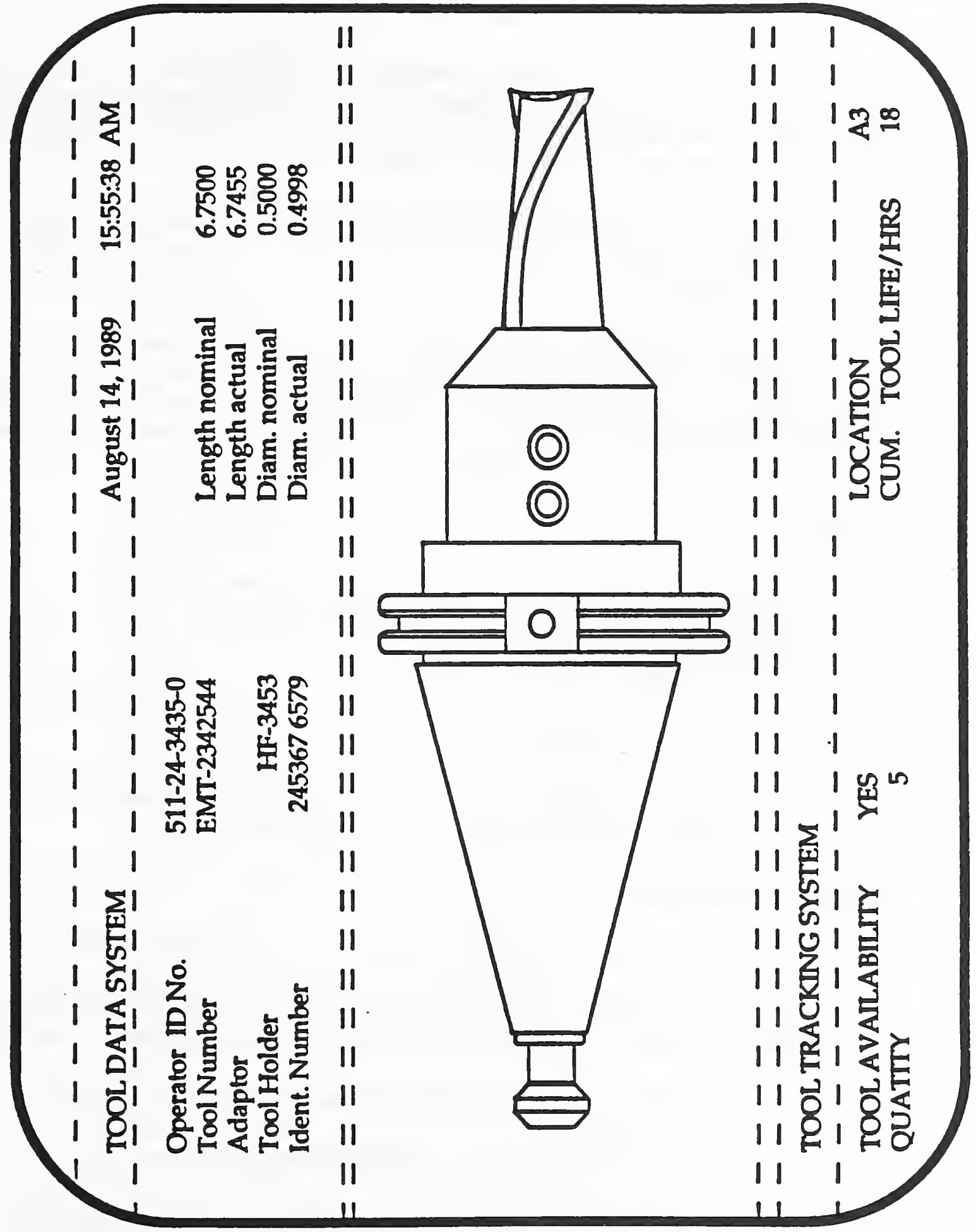

Figure 4-3. Example terminal display of tool setup specification 


\subsection{Component Inventory Management}

Inventories of consumable and reusable tool components are tracked to predict reorder points and determine consumption rates. The data base is updated every time components are withdrawn from or returned to stock. Tool assemblies are not tracked within this system, only individual components are tracked.

Component inventory data will be required by CIMS. A CIMS link will need to be established to this local data base. This link could be in the form of printed reports or a network connection.

The TMS will provide data terminals, user interface software, and data base management software to coordinate tooling component inventory control within the tool room. The TMS will use these terminals both to provide data to tool room personnel and to acquire data from the staff. The TMS must manage the component inventory data base and provide for data input from several terminals.

\section{Functions}

* Maintain and control tooling component inventory

* Maintain a tooling component inventory data base

* Identify stock which is at or below reorder point

* Generate usage levels for tooling

Timing requirements for performing functions

* Inventory transactions - the response to the withdrawal or return of a tooling component should be less then 10 seconds.

\section{Hardware requirements}

* Two terminals shall be provided in the component storage area.

* The user interface provided on these terminals shall be support data entry and display operations for $a^{*} t$ least the following types of activities:

- Determine the availability and location of components in inventory.

- Record withdrawal and return of components from and to the inventory.

- Record the arrival of new components to the inventory. 
- Search the data base on the basis of inventory parameters.

- Generate tool room exception reports.

- Initiate the printing of hard copy reports.

* Inventory parameters shall include but not be limited to:

- tool number

- description

- tool name

- manufacturer

- supplier

- identification number

- storage location

- quantity on hand

- quantity on order

- reorder point

- reorder quantity

- price per unit

- date last used

- order number

- diameter

- shank type

- cutter type

- flute length

- overall length

- number of flutes

- surface treatment

* Data base search routines shall include, but not be limited to:

- tool number

- manufacturer

- supplier

- storage location

- type

- size (diameter, length)

- surface treatment

- reorder point reached

* Hard copy reports that shall be printable on the tooling inventory printer shall include, but not be limited to: tooling component inventory, inventory 
reports by parameter, components at reorder point, and an outstanding exceptions summary.

* One printer shall be provided for shared access by the user terminals in the component storage area.

* A bar code system for component inventory management should be considered.

* Disk storage of component inventory and should be copied daily to tape (or other suitable media). The backup disk and tape storage system should be equipped with an uninterruptible power supply capable of maintaining data base integrity in the case of a power failure.

\section{System inputs}

* Components returned to inventory $(T)$

* New components added to inventory (T,D)

* Tool room exception reports (T)

* Operator display selections (T)

* Operator data base search requests (T)

* Operator hard copy report requests (T)

System outputs

* Components withdrawn from inventory $(E)$

* Tool inventory reports (T,D,P)

* Reorder reports (T,D,P)

* Data base backups (D,P)

\section{Discussion}

The component inventory management system shall maintain a real time account of locations and quantities of tooling components in the tool room. All withdrawals and returns of tooling components shall be recorded by entering the required data at a terminal or a bar code reader station.

The component inventory system represents a definite interface point to CIMS. The CIMS TCM module will require the data maintained in the local component inventory management system. This CIMS link could take the form of a network interface or it could be a simple printed report. The exact nature of this link cannot be determined until the CIMS TCM module is completely defined. 


\subsection{Assembly Storage, Issue and Receipts}

The TMS will provide data terminals and user interface software to coordinate issue and receipt operations within the tool room. The TMS will use these terminals both to provide data to tool room personnel and to acquire data from the staff. The TMS must also provide interfaces for data acquisition to the tool identification system.

Completed tool assemblies, arriving from Assembly and Presetting, are stored in local storage racks. Tool assemblies are inspected before they are issued. Tool assemblies are issued by wanding the tool identification (e.g., the tool identification chip). The TMS then automatically updates the tool data base to show that the tool assembly has been released to the transport and delivery system.

Tool assemblies are received from production by wanding the tool identification chip. Tool assemblies are placed in local storage racks as they are received from production. They are stored separately from the tool assemblies which are awaiting issue. The returned tool assemblies are either sent to presetting for rework, or they are disassembled and their components are returned to storage.

Tool assembly issue and receipt data is only relevant to the local tool management system. It is not required by CIMS.

\section{Functions}

* Control the issue and receipt of tool assemblies.

* Read tool identification and verify that only one data file exists in the tool assembly data base for the identification code.

* Update the tool assembly data base as tools enter/exit the tool room.

* Verify tool assembly configuration before issuing the tool assembly to production.

* Load tool assemblies onto tool assembly transport devices. The operators should not be required to place tool assemblies in predetermined locations on the transport device.

Timing requirements for performing functions

* Tool assembly data base transactions - Data base transactions should be completed within 10 seconds of an operator initiating the transaction. 
* Tool identification data - Data acquisition from the tool identification system (micro-chip reader stations) should be completed within 3 seconds after operator indicates that tool identification data is ready for acquisition.

\section{Hardware requirements}

* Two data entry and display terminals shall be provided local to the issue and receiving area.

* The user interface provided on these terminals shall be support data entry and display operations for at least the following types of activities:

- Issue a tool assembly

- Receive a tool assembly

- View a tool setup specification

- Display a tool assembly drawing

- Report the status of a tool assembly

- Generate tool room exception reports

- Initiate the printing of hard copy reports

* Two tool identification readers shall be provided to the issue/receiving area. Suitable hardware interfaces shall be provided for the purpose of communicating acquired data from the tool identification system.

System inputs

* Tool assembly received $(T, R)$

* Tool setup schedule (D)

* Tool identification (R,T)

* Tool transporter identification $(\mathrm{R}, \mathrm{T})$

* Tool setup specification (D)

* Tool assembly drawing (D)

* Data acquisition status indication (R)

System outputs

* Tool assembly issued (D,E)

* Tool transporter rack issued (D,E)

* Operator display selections (T)

* Operator hard copy report requests (P)

* Tool room exception reports (D,T,P) 


\section{Discussion}

The issue and return of tool assemblies to the tool room must be tightly controlled. Each transaction must be recorded by reading the tool identification chip in the tool holder. The data base must then be automatically updated to reflect changes in the status of a tool assembly. An audible and a visual conformation must be given to the operator that this data base transaction has occurred. The TMS shall provide a graphics terminal and a tool identification reader to the issue and receiving area of the tool room to support issue and receiving activities.

Each tool assembly is identified by the tool identification number. When tool assemblies are issued, the tool identification chip is wanded. An unmatched identification number must cause a fault signal to be issued to the operator in an audible form. An error message should be displayed on the CRT. Once a tool assembly is identified, it may then be verified by calling up the tool assembly drawing and comparing it with the actual assembly. The operator should then verify that tool offset data have been recorded for this tool assembly. Tool assemblies should not have to be placed in specific positions in the tool transport device. Specific position assignments would increase the possibility of operator error. Since tool assemblies are automatically identified as they enter a cell, specific position assignments in the transports are unnecessary.

Tool assemblies returned from the production area will be identified and placed in temporary storage. These tool assemblies will be inspected then reworked or disassembled.

Tool assembly issue and receipt data is not relevant to CIMS except as it pertains to the status (location) of tooling components within the system. The data on individual tool assemblies is only required for the local tool management system.

\subsection{Inspection and Disassembly}

Tool assemblies that are no longer needed on the shop floor must be returned to the tool room. Worn tool assemblies must also be returned. Tool assemblies that are no longer needed must be disassembled and their component parts returned to stock, sent to regrind, or scrapped. Tool assemblies are no longer needed when the part(s) they are used on will not be manufactured within the next 30 days.

The component inventory is updated when tool components are returned. Tool assemblies that require rework (e.g., worn consumables replaced) are transferred to 
assembly and presetting, reworked, and then reissued. Worn consumables that can be reused are sent to the regrind area.

The TMS will provide data terminals and user interface software to coordinate the routing of tool assemblies returned to the tool room. The TMS will use these terminals both to provide data to tool room personnel and to acquire data from the staff. The TMS must also provide interfaces for data acquisition to a planned tool identification system.

\section{Functions}

* Identify and determine the disposition of tool assemblies returned to the tool room.

* Disassemble tool assemblies that are no longer required for production and return individual components to component inventory.

* Update the component inventory data base when components are returned to inventory.

* Route tool assemblies that are required for production to the presetting area for rework.

* Route worn (dull) cutting tools to the regrind area.

Timing requirements for performing functions

* Tool data base transactions - Data base transactions should be completed within 10 seconds of an operator initiating the transaction.

* Tool identification data - Data acquisition from the tool identification system should be completed within 3 seconds after operator indicates that tool identification data is ready for acquisition.

\section{Hardware requirements}

* Two data entry and display terminals shall be provided local to the inspection and disassembly area. 
* The user interface provided on these terminals shall support data entry and display operations for at least the following types of activities:

- Request the status of a tool assembly

- Report the status of a tool assembly

- Generate tool room exception reports

- Initiate the printing of hard copy reports

* One tool identification reader shall be provided to the inspection and disassembly area. Suitable hardware interfaces shall be provided for the purpose of communicating acquired data from the tool identification system.

System inputs

* Tool identification (R,T)

* Tool identification reader status indication (R)

* Tool setup schedule (D)

* Tool setup specification (D)

* Tool assembly drawing (D)

* Tool availability report (D)

* Tool assembly data and status ( $T, R, D)$

System outputs

* Operator display selections (T)

* Operator hard copy report requests $(\mathrm{P})$

* Tool assembly status update, e.g., returned for regrind, no longer required, routed to presetting, etc. (T,D,P)

* Tool room exception reports entered $(T, D, P)$

\section{Discussion}

The routing of returned tool assemblies inside the tool room must be rapid and accurate. Each transaction must be recorded and the data base must be immediately updated to reflect changes in the status of a tool assembly. A terminal and a tool identification reader, e.g., a wand, should be provided for installation in the inspection and disassembly area of the tool room. This station will support the routing of tool assemblies and tooling components.

Each tool assembly is identified by the tool identification number. Tool assemblies will be identified by reading the tool identification. The status of a tool assembly may 
then be checked by accessing the tool assembly data base to determine the proper routing for the tool assembly.

Tool assemblies that require rework (worn consumables replaced, inserts indexed, etc.) will be sent to presetting and then reissued. Tool assemblies that are no longer required (for the next 30 day period there is no part calling for this tool) will be disassembled and their component parts returned to inventory. All worn consumable components will be sent to regrind or scrapped. All monitored tools are separated and set aside for the tool monitoring program personnel.

It is essential that the tool data base be updated when tool assemblies are reworked or disassembled. All tool setup data associated with an identified holder must be removed prior to the holder being returned to inventory for future use. Most tool setup data must be removed prior reworking a tool assembly. This should be done only after pertinent information is extracted and stored in historical data files for future analysis.

\subsection{Tool Regrind}

The tool regrind areas is responsible for reconditioning worn tools. Worn tools, e.g., drills, mills etc., are redressed, inspected and returned to the component inventory.

\section{Functions}

* Recondition worn cutting tools for reuse in production operations.

* Report quantities of cutting tools reconditioned, quantities scrapped. * Update component inventory data base when cutters are scrapped.

Timing requirements for performing functions

* Data base transactions - The response to a data base update should take no more than 10 seconds.

Hardware requirements

* One data entry and display terminal shall be provided local to the regrind area.

* The user interface provided on this terminal should be the same as the terminals for the component inventory terminals. 
System inputs

* Worn cutter identification data (T)

* Worn cutters (E)

System outputs

* Reconditioned cutting tools (E)

* Component inventory updates (D,T)

* Tools processed reports $(\mathrm{P}, \mathrm{D})$

Discussion

An accurate record of scrapped tooling must be maintained to assure that replacement tooling can be procured in a timely manner.

\subsection{Performance Monitoring}

Tool monitoring data for some tool assemblies is collected as these tool assemblies pass through the tool room. Information pertaining these tool assemblies (e.g., condition of tool point after regrind) must be recorded to the tool data base. The TMS will provide data terminals and user interface software to input tool monitoring data to the tool data base. The TMS will use these terminals both to provide data to tool room staff and to acquire data from the tool room staff. The TMS must also provide interfaces to a planned tool identification system.

\section{Functions}

* Collect tool monitoring data as tool assemblies are processed through the tool room.

Timing requirements for performing functions

* Tool data base transactions - Data base transactions should be completed within 10 seconds of an operator initiating the transaction.

* Tool identification data - Data acquisition from the tool identification system should be completed within 3 seconds after operator indicates that tool identification data is ready for acquisition. 


\section{Hardware requirements}

* One data entry and display terminal shall be provided local to the tool monitoring station.

* The user interface provided on this terminal shall be support data entry and display operations for at least the following types of activities:

- Check the current tool status (location, remaining life, number of regrinds)

- Input tool data (life between regrinds, regrind parameters, visual inspection comments, etc.)

- Initiate the printing of hard copy reports

* One tool identification reader shall be provided local to the tool monitoring area. Hardware interfaces shall be provided for the purpose of communicating acquired data from the tool identification system.

System inputs

* Tool monitoring data $(T, D)$

* Tool Identification (T,R)

* Data Acquisition Status Indication (tool identification) (R)

System outputs

* Tool monitoring data $(\mathrm{T}, \mathrm{D}, \mathrm{P})$

* Operator display selections (T)

* Operator hard copy report requests $(\mathrm{P})$

* Tool assembly status data $(T, D, P)$

\section{Discussion}

An important part of the tool monitoring function is collecting life cycle data on tooling used in production. This tool wear analysis data is collected by tracking selected tools through their life cycle and recording performance data. Performance data is collected at the machine tool with the Freeze Frame system and tool wear sensors. Performance data is also collected in the tool room by visual inspection before and after regrind.

In the tool room, selected tools that have been specially identified are separated from the normal tool flow. Data concerning these tools, e.g., number of regrinds, life 
between regrinds, parameters of regrind, etc., are recorded in the tool data base. These tools are subject to handling restrictions inside the tool room. Each tool must remain with the same tool holder, i.e., it must maintain the same tool identification number, until that tool is scrapped. Each tool must be held for special inspection before and after regrind. (The above discussion is a description of how performance monitoring relates to tool room activities only - a further discussion of tool monitoring is found in Section 6). 


\section{Tool Assembly Storage and Delivery}

Tool assembly transport involves the physical exchange of tool assemblies between storage locations in the system. A bidirectional flow exists between the tool room and the various machining cells in the production area to ensure availability of tools when needed. If tool transfer is accomplished manually, tool kits are prepared in the tool room for each job or batch. These kits are sent to the appropriate machining cells on a trolley, forklift, or by other means. Unscheduled tooling requests are also filled in the same manner. Worn tools are sent back to the tool room where appropriate action is taken, e.g., regrind, replace inserts, etc.

The transfer of tools between the tool room and the machining cells may be automated in various ways. Exchanges may involve individual tool assemblies, a small cartridge of several tools, or an entire machine tool magazine. The segmented magazine approach is a compromise between the flexibility of swapping individual tools and the simplicity of swapping entire magazines, which saves the transport network from overload at the expense of flexibility (e.g., transferring a tool assembly between two machines) and a higher tool inventory. The type of system used is a function of lot sizes and part variability. With large lot sizes, "bulk" exchange of tooling would be adequate. With small lot sizes and diverse part types, more flexibility is required and a system that can handle individual movement of tool assemblies has many advantages.

There are two basic types of automated transport systems; overhead cranes and automated guided vehicles. Overhead cranes are usually used to transport part or all of a machine tool magazine. They are much less complicated than AGV systems and, in general, cost less. AGV type systems can also be used transport to tool cartridges, but have the added flexibility required to route individual tools (or groups of individual tools) through the system. With AGV systems, the tool handling network should be independent of the work piece handling network. The use of the material handling system to transport tools often interferes with random movement of work pieces and complicates material flow planning.

Tool storage buffers are placed next to machine tools or groups of machine tools to complement the tool storage capacities of the individual tool magazines. These buffers come in the form of stationary shelves, racks, or moveable carousels. Some storage units are vertical, others are horizontal. They may be totally separate from the machine tool or integrated with the machine and its controller. A pick-place manipulator may be used to facilitate the tool transfer between the buffer and the tool magazine on the machine tool. This manipulator is a simple device which can be attached to the machine tool itself. A larger, mobile robot may be used to transfer tool assemblies between individual machines in machining cells where several machine tools are grouped together. 
The ability to respond automatically to scheduled tool requirements, as well as unexpected tool requests, necessitates the use of an automated storage and retrieval system (ASRS) for tool assemblies in the tool room. This ASRS must be computer controlled and integrated with the rest of the automated manufacturing system to enable it to receive, interpret, and respond to requests for tools. Completed tool assemblies are stored in the ASRS according to production plans and estimates of unexpected failures. Tools may be stored in the ASRS individually or in groups according to the adopted transfer control method. The storage location of all tool assemblies must be maintained in the tool data base.

A computer controlled ASRS is a prerequisite for implementing unmanned machining operations, regardless of the level of automation of the other activities in the tool room. With a manual tool delivery system, the ASRS can be controlled by an operator from an adjacent terminal. The storage capacity of the ASRS required for a given tool management system can be determined using computer simulation.

\subsection{Automated Tool Assembly Delivery}

The System Controller will issue commands to the tool delivery automated guided vehicle control system. TMS will provide data terminals to display operations of the tool delivery AGV system and provide manual control when necessary. The tool delivery AGV system will be capable of transporting tool assemblies between the tooling automated storage and retrieval system and the tool que stations of zones $A, B, C, D$ in the automated manufacturing area.

\section{Functions}

* Deliver tool transport devices to machining cells.

* Transmit AGV status and location reports to tool management system controller.

Timing requirements for performing functions

* User data entry - (See Section 8.4)

* Display terminal - (See Section 8.4)

* Command acknowledgement - Commands received from the tool management system controller should be acknowledged within 5 seconds after they are received. 
Tool Assembly Storage and Delivery

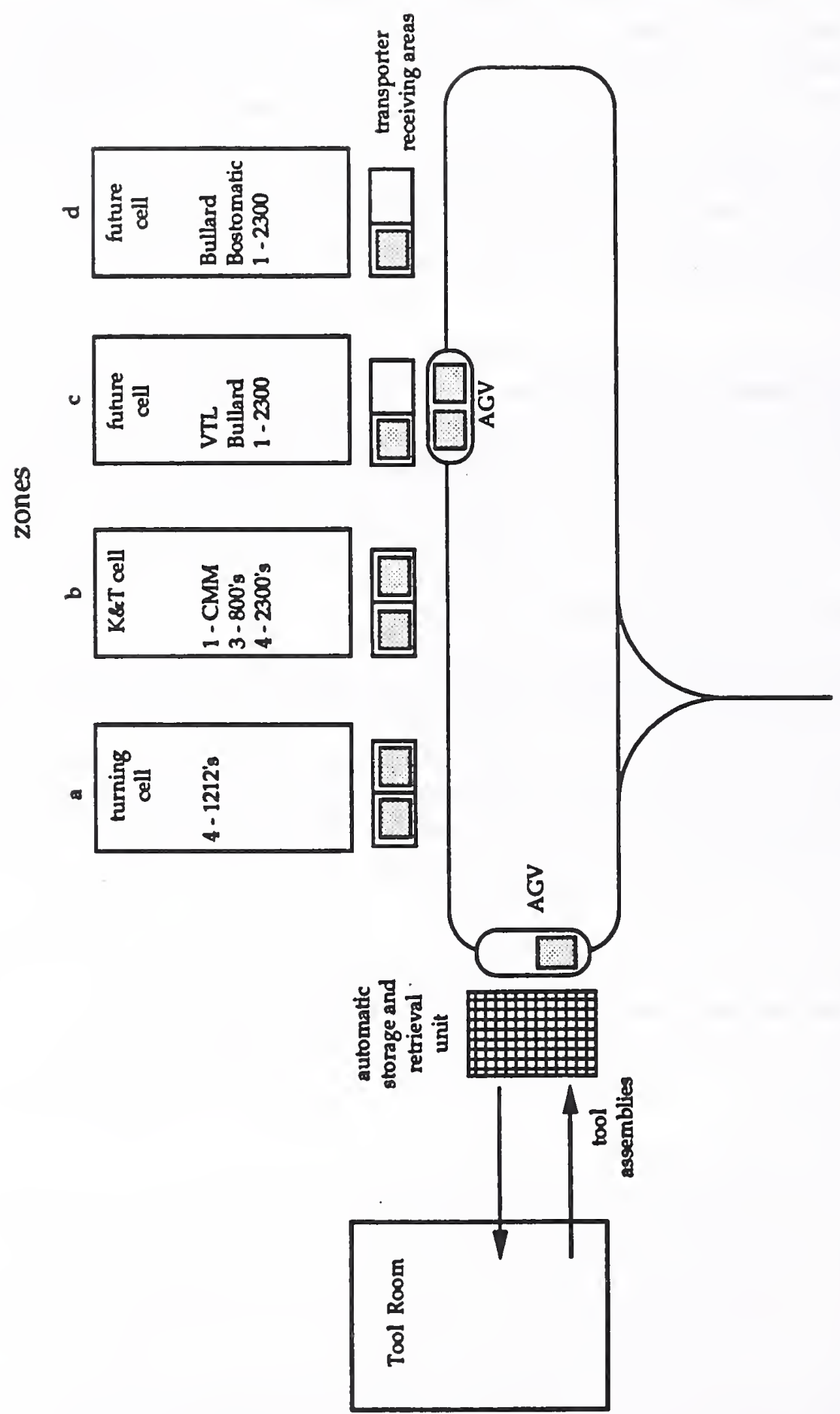

Figure 5-1. Automated tool assembly delivery system 
* Vehicle performance - The AGV system should be capable of making one round trip drop-off/pick-up operation between the tool room and zones $A-D$ in no more than 15 minutes.

\section{Hardware requirements}

* At least two AGV's and associated hardware are required.

* Tool transport devices should be capable carrying a minimum of 12 tool assemblies.

* One terminal should be provided for AGV console purposes. See Section 8.4 for terminal requirements.

* The AGV controller should be equipped with several communications interface options such as; Ethernet, MAP, RS-232, RS-422.

* Load Carrying Capabilities. The vehicle should be equipped to accept the tool assembly transfer device specified for the system. The vehicle should be capable of carrying a minimum load of 3000 pounds. The load may be unevenly distributed.

* Vehicle Guidance. The vehicle should be capable of following a wire, taped to or buried in the floor, over which a guidance signal is transmitted. The wire sensor should not require contact with the floor.

* The vehicle should be capable of stopping at locations to load or unload materials. These locations should be defined by data input to the control computer (this is a very important point - no "hard-wired" load and unload points should be allowed). Changing of the station locations should not require modifications to the control software but only to input data (no "hard-coded" load and unload points should be allowed). The vehicle should be capable of stopping at these locations with an acceptable tolerance (this depends on transfer mechanism). The vehicle should also be capable of passing by these locations without stopping if no load transfer is required there. The vehicle should stop as soon as the guidance signal is lost or stopped, unless specifically commanded to do otherwise. The vehicle should be capable of switching to alternate guide paths when commanded to do so. The vehicle should be capable of operating on the same guide path at the same time, with other identical vehicles. 
* Vehicle Operation. The vehicle should be capable of operating in both manual and automatic modes. In the manual mode, an operator should be able to guide the vehicle to any desired location through the use of a hand held control box. The operator should also be able to control the speed of the vehicle and the auxiliary functions including the load transfer and battery charging mechanisms. In the automatic mode, the vehicle should follow the guide path wire and perform functions it is commanded to do without human intervention. The vehicle should be capable of operating in a multiple vehicle system environment. The vehicle should be capable of operating in both forward and reverse directions in both manual and automatic modes. The vehicle should be capable of reversing direction at any point on the guide path.

System inputs

* Tool transfer commands received in manual or automatic mode (T,S)

* Tool transporters from tool room and machining cells (E)

System outputs

* Tool transporters to machining cells and tool room (E)

* Command acknowledgement $(T, S)$

* Tool assembly transfer device status $(T, S)$

* Vehicle status and location (T)

* AGV Status Reports of daily performance (P)

\section{Discussion}

The installation of and automated tool assembly delivery system (AGV and ASRS) is the last step in the staged implementation of the TMS. An automated tool assembly delivery system should be implemented only after all other tool management systems are installed and fully functional. A manual delivery system will be fully adequate in the interim. Since the communications interfaces and the command structures will be virtually identical for both the automated and manual systems, conversion to an automated system in the future should not require any major modifications to the tool management system.

\subsection{Automated Storage and Retrieval of Tooling}

The tool assembly automatic storage and retrieval system will be used in an integrated tool delivery system which includes automatic guided vehicles. The ASRS will 
store tool transport devices and transfer them to and from AGV's. Interaction between the Tool Room and the ASRS will be manual. The system will automatically transfer tool transporters (tray, chain, rack, etc.) onto and off of an AGV. The system should respond to storage and retrieval instructions for storing and transferring tool transporters. The ASRS controller will accept instructions entered manually or transmitted from the tool storage and transport control computer.

Functions

* Store tool transport devices.

* Transfer tool transporters to and from AGV's.

* Transmit ASRS status and contents reports to tool management system controller.

* Identify tool transport devices.

Timing requirements for performing functions

* User data entry - (See Section 8.4)

* Display terminal - (See Section 8.4)

* Command acknowledgement - Commands received from the tool management system controller should be acknowledged within 5 seconds after they are received.

* System performance - The ASRS system should be capable of responding to commands without delay, and capable of stopping on demand. Tools should be delivered within scheduled times provided by schedules produced in the system.

\section{Hardware requirements}

* The system should consist of identical modules for storing tool assembly transport devices (e.g., trays, .racks, etc.) on shelves. The system should consist of the necessary components and other supporting/auxiliary systems necessary to operate manually and automatically as an integral unit. It should perform operations under computer control or under operator control.

* Control System. The ASRS should include a local control computer capable of controlling the system and responding to commands from and providing status to an external supervisory control computer. It should be 
possible for an external computer to control the actions and maintain status communications with the storage system control by network interface. The system should respond to external computer commands to : 1) transfer trays between selected shelves and the conveyor, and 2) transfer a tray between the conveyor and the AGV. Sensors should detect the presence of the AGV and disable tray transfer off the conveyor if the AGV is not present. The system should provide status feedback to the external computer. The status data should indicate whether the system is busy with a current operation or whether it is ready for another command. Error conditions should be reported in the status data. Manual intervention should be possible.

* Manual Operation. Activation of all specified functions should be possible by manual operation from a terminal.

* Tray Identification System. The system should include a means for automatically identifying tooling transport devices. Each transport device should incorporate a permanently attached electronic or optical identifier which can be read automatically. Read heads should be installed in all locations where tray transfers take place. Hardware, software and communications interfaces should be provided for the purpose of reading and communicating transport device identity to the system controller.

* One terminal should be provided for ASRS control purposes. See Section 8.4 for terminal requirements.

System inputs

* Tool transporter transfer commands received in manual or automatic mode $(\mathrm{T}, \mathrm{S})$

* Tool transporters from AGV's (E)

System outputs

* Tool transporters to AGV's (E)

* Command acknowledgement (S,T)

* ASRS status (T,S)

* ASRS contents report (T,D,P) 


\section{Discussion}

The ASRS referenced here is not the existing ASRS (Interlake) and is not the existing Stanley-Vidmar stacker presently located in the tool room. This ASRS will act as a buffer between the tool room and the automated tool delivery system. Completed tool assemblies that have been inspected and issued to production are placed in the ASRS and are then considered "in the system." All interaction between the AGV and the ASRS is completely automated.

\subsection{Manual Handling and Storage}

The TMS will provide data terminals and user interface software such that total manual delivery and storage of tool assemblies is possible before an automated tool delivery system is implemented. The terminals should provide display software that is human readable for manual activities.

\section{Functions}

* Display terminals shall be provided local to the tool room, and tool storage area.

* The user interfaces provided for these terminals should support the following activities:

- View tool delivery requests

- View contents of tool storage areas

- View list of tooling to be removed from cells

* All commands shall be accessible from the console computer and all operations shall be able to be performed manually.

* The tool delivery controller will make all tool movement decisions. The operator need only read tool delivery requests and perform the required actions to satisfy these requests.

Timing requirements for performing functions

Controller Data - The system shall send commands to the operators terminal as soon as a previous command has been accepted and/or executed. 
Hardware requirements

Console terminal - A console terminal shall be provided for operator control of the storage systems. All reports and status formats shall be accessible from this console. Any intervention shall also be done from the console.

System inputs

* Operator display selections (T)

* Operator hard copy report requests (T)

* Tool identification $(T, R)$

* Tool transfer commands (T,P)

System outputs

* Tool Allocation Schedule (P,T)

* Tool Availability Report (D,P,T)

\section{Discussion}

The installation of an automated tool assembly delivery system (AGV and ASRS) is the last step in the staged implementation of the TMS. An automated tool assembly delivery system should be implemented only after all other tool management systems are installed and fully functional. A manual delivery system will be fully adequate in the interim. Since the communications interfaces and the command structures will be virtually identical for both the automated and manual systems, conversion to an automated system in the future should not require any major modifications to the tool management system. With a manual tool delivery system, tool transport devices will be delivered by forklift to tool transport device receiving area in each zone. Tool storage would also be manual (i.e., the Stanley-Vidmar stacker which currently resides in tool crib). 


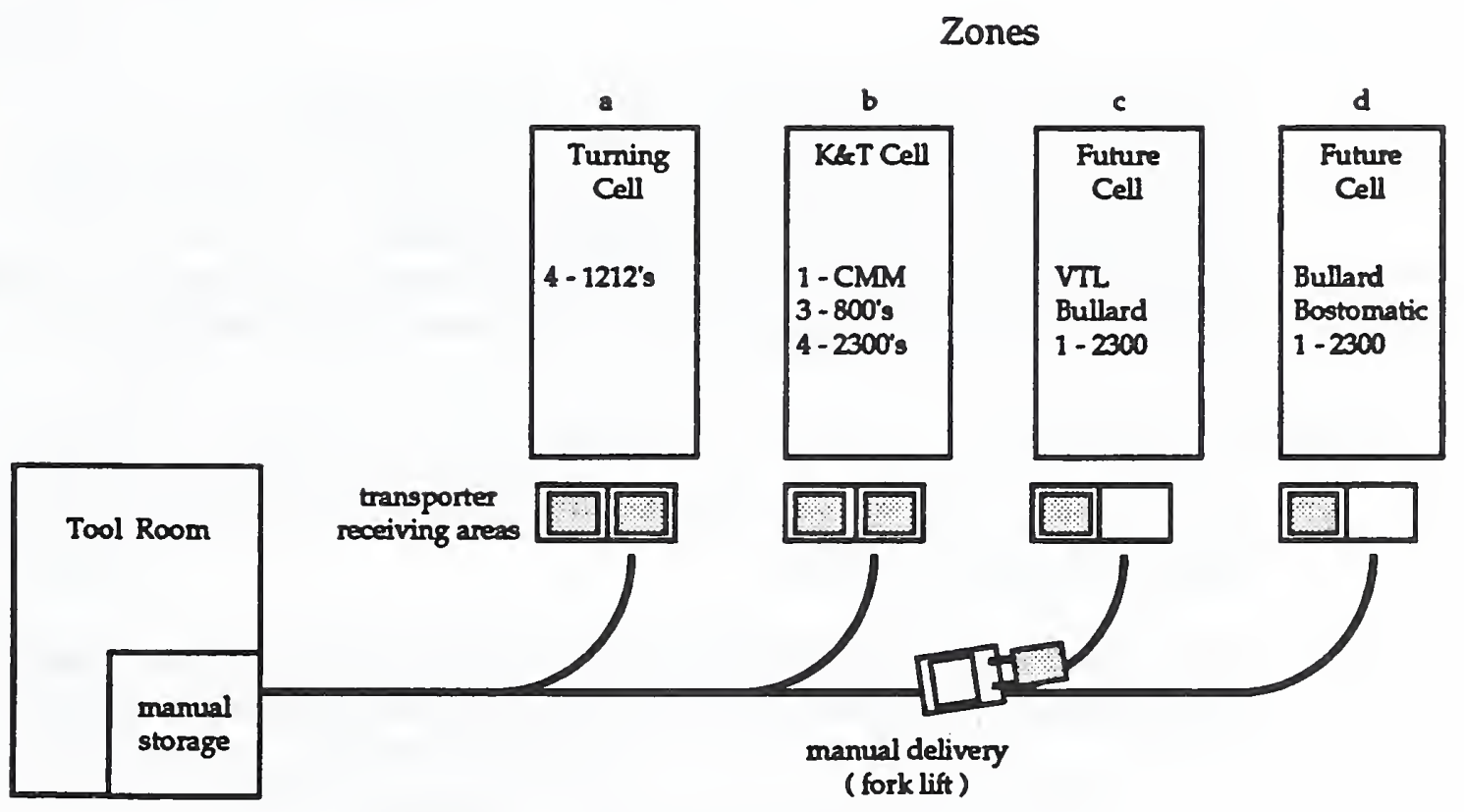

Figure 5-2. Manual tool assembly delivery 


\subsection{System Controller}

The TMS will provide communications links, interface software and user interface terminals to the tool delivery system controller. The tool delivery system controller coordinates the activities of the AGV and ASRS. The controller must constantly track the location of the AGV's and all the tool transport devices (racks or trays delivered by AGV's). The controller must also maintain an up-to-date record of the contents of the ASRS.

\section{Functions}

* Coordinate the activities of the tool delivery system.

* Plan the movements of the AGV's.

* Transmit move requests to the AGV controller.

* Transmit move requests to the ASRS controller.

* Report delivery system status to the tool management system controller.

* Update tooling data base when tooling enters/exits ASRS.

\section{Hardware requirements}

* One data entry and display terminal shall be provided at the tool delivery system controller.

* The user interface provided on this terminal shall be support data entry and display operations for at least the following types of activities:

- Check the current tool assembly location status.

- View an AGV Status Report.

- Display an ASRS Status Report.

- Determine the availability and location of storage areas in ASRS.

- Generate Tool Transfer Reports.

* All transport system controller commands shall be accessible from the console computer and it should be possible to perform all operations in both manual and automatic modes.

* The tool delivery controller must have a suitable communications interface with the tool management system controller and the tool management data bases. It is possible that the tool delivery controller could be a process running on the same computer as the tool management 
system controller. If it runs on a separate computer, communications should be over the local network.

Timing requirements for performing functions

* Controller Data - Shall be able to send commands to the AGV or ASRS systems as soon as a previous command has been accepted and/or executed. The system should be immediately ready to respond to the next request after completing a transaction.

System inputs

* Tool delivery commands from TMS controller (S)

* Tool Availability Report (D)

* Identification of tool transport device removed from ASRS by tool room $(R)$

* Identification of tool transport device placed in ASRS by tool room $(\mathrm{R})$

* Report on contents of tool transport device (D)

* Operator display selections (T)

System outputs

* Command acknowledgements to TMS controller (S)

* Tool transfer commands to the AGV and ASRS systems (E)

* Tool delivery system status (T)

* Operator hard copy reports (P)

Discussion

The tool delivery system controller is responsible for directing the actions of the AGV and ASRS. The local controller would receive tool transfer commands from the TMS and would then determine the required AGV and ASRS commands to accomplish the required tool transfer. The controller would issue these commands to the equipment controllers and monitor their actions. A local system controller will not be required unless both an ASRS and AGV system are present. Dispatches for manual moves can be issued to terminals directly by the TMS Controller. 


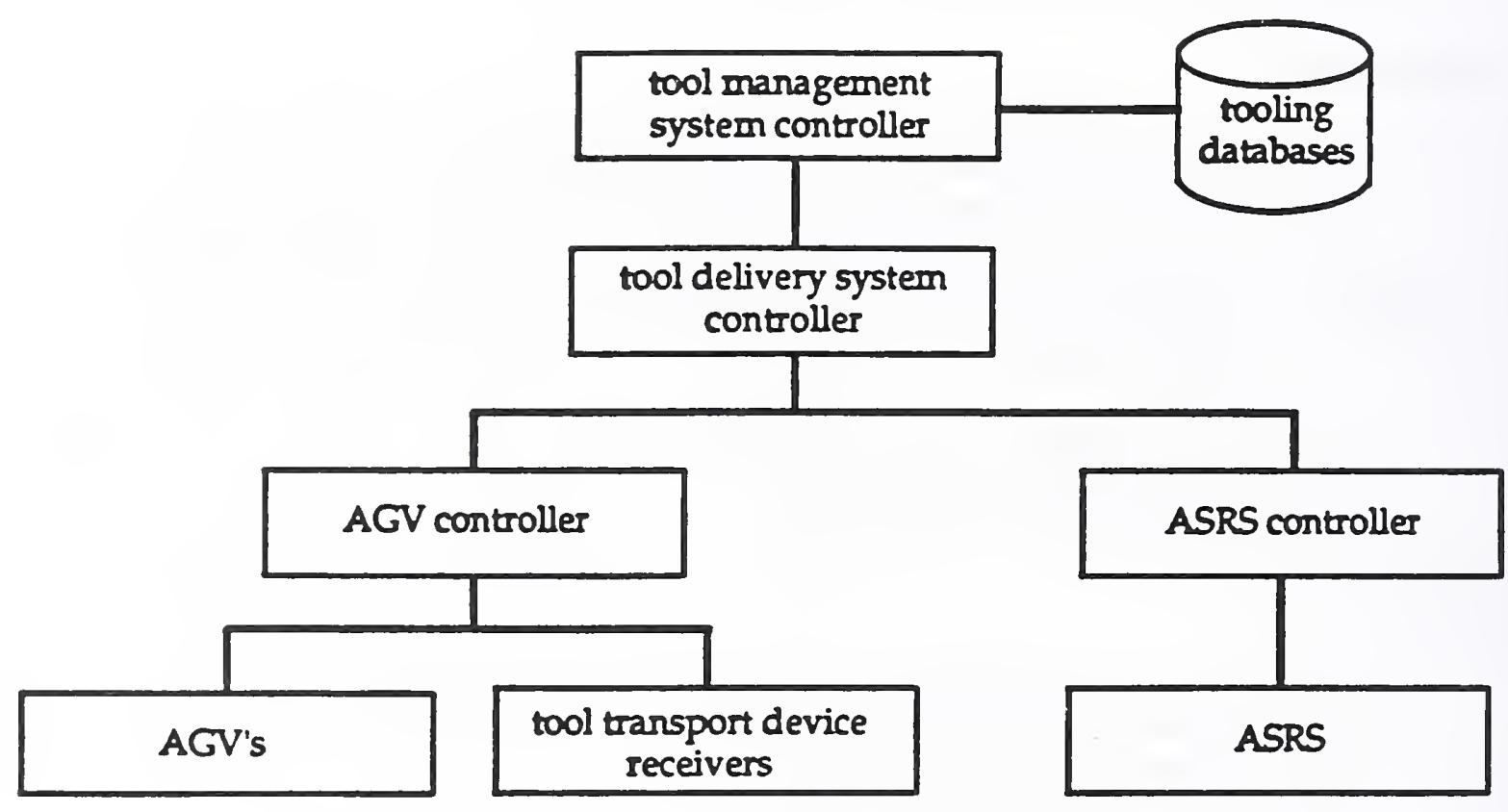

Figure 5-3. Block diagram of proposed automated system 


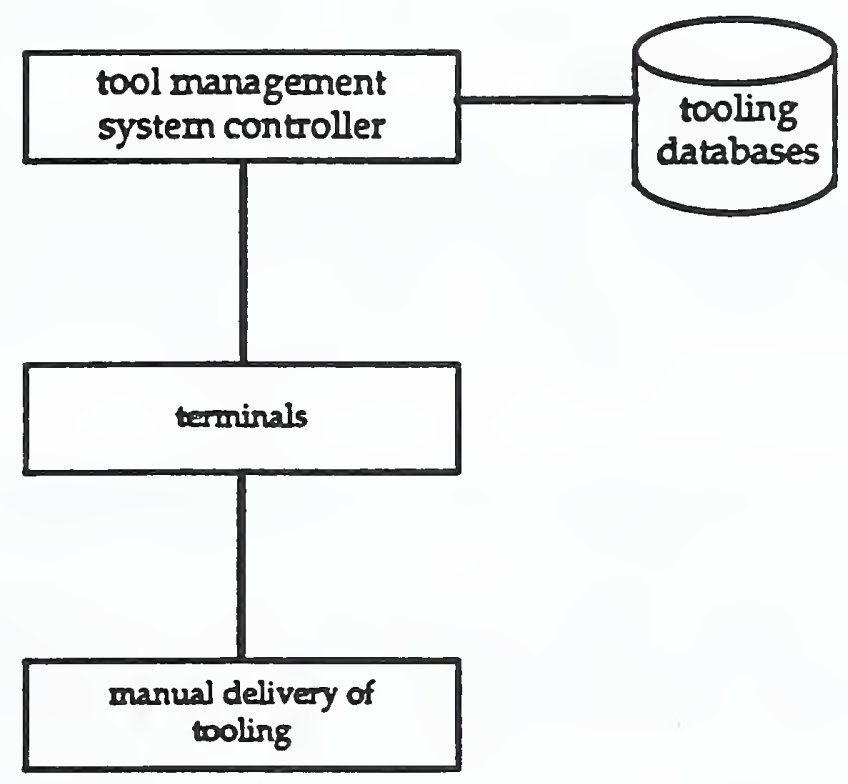

Figure 5-4. Block diagram of proposed manual delivery system 


\section{Tool Tracking and Data Collection}

The performance of cutting tools is one of the most critical factors governing the productivity of machining operations. Tool identification systems are needed to track tools through the system and to prevent an incorrect tool from being used. Sensors are needed for monitoring tool wear (in order to initiate tool replacements), and for detecting sudden breakage to prevent further damage to the work piece or machine. The system should be able to detect both imminent and actual tool failure. It should be adaptable to support a wide range of machine tools.

\subsection{Tool Identification System}

The tool identification system provides for unique identification of each tool assembly in the automated manufacturing system domain. This unique identification is used by the tool tracking system to identify the tool data base entry that is associated with a particular tool assembly.

\section{Functions}

* Provide each tool holder with a unique identification chip capable of supporting a minimum of a 32 -bit identification code.

* Provide reader heads (stationary and wand type) and reader controllers at various locations throughout the system; machine tools, buffer storage locations, tool room, etc. (see section 6.2).

* Provide communication interfaces between reader controllers and network interfaces, CRT displays, terminals, machine tool controllers, cell controllers and host computers.

Timing requirements for performing functions

* Read rates - Read rate must be less than $8.0 \mathrm{~ms}$ with $\pm 5.0 \mathrm{~mm}$ misalignment.

* Verification - Readers must perform a minimum of five scans per "read" to provide verification of tool assembly identification.

* Tool magazine reads - The system must be capable of reading with relative motion (moving tool magazine). Relative motion limit should be no less than $25.0 \mathrm{in} / \mathrm{sec}$. 


\section{Hardware requirements}

* The tool assembly identification system should be the read-only noncontact microchip type.

* Readers should be capable of non-contact sensing up to an $8.0 \mathrm{~mm}$ read distance.

* Code carrier should be imbedded in the tool holders.

* No less than 1000 identification code carrier chips should be provided with the system.

* The memory capacity of each code carrier should be a minimum of 32 bits.

* Communication between the microchip and reader head must not be impaired by oil, dirt, cutting fluid, or metal chips.

* Data exchange must be complete and accurate. Reader reliability must be on the order of $99.9 \%$ success.

* Reader controllers should be capable of accepting data from no less than four read heads and communicating the data to the host computer (e.g., cell controller).

* Reader controllers must generate a read error message when a read error occurs.

* The system must not be affected by extraneous radio frequency energy or electrical noise.

* Read heads must be small enough to mount in machine tool magazines.

* Hand held readers should be available for manual identification of tool assemblies.

System inputs

* Message receipt acknowledgment to reader controllers (D,S) 
System outputs

* Tool assembly identification (D,S,T)

* Tool transport device identification $(D, S, T)$

* Read error messages (S,T,P)

\section{Discussion}

Automatic tool identification systems consist of identification tags mounted on the tool holders, readers that communicate with the tags, and reader controllers that collect data from the tags and transmit the data to system computers that store and interpret the data. An effective tool identification system is a preliminary requirement to a tool management system. The tool identification can point to a data base that contains such information as storage location, tool offsets, remaining life, cutting parameters, and sister tools. To eliminate the great potential for human error in manual record keeping and keyboard data entry, the technologies of barcoding and embedded integrated circuits have been adopted for use with cutting tools. These technologies permit error-free storage and transfer of tool information tool room, machine, and computers.

All automatic tool identification systems fall into two major categories: read-only and read-write. In read-only systems, the tags carry fixed codes only. Readers acquire data from the tags, but cannot transmit to them. In read-write systems, the tags contain programmable memory chips. Variable data are transmitted from the local controller, through a reader, to an individual tag. When excited by signals from a read head, the tag transmits its data back through a reader to the controller.

Within the two major categories of tool identification systems there are four basic technologies. These are 1) bar codes, 2) microchips with (IC) induction coils, 3) microchips with (RF) radio frequency transceivers, and 4) microchips with mechanical contacts.

Mechanical contact type systems are undesirable because the tool assembly must be stationary to perform the read operation. This would result in unacceptably slow read rates. Bar code systems are undesirable because the bar code tags are susceptible to damage and contaminate build-up which would result in a unacceptable level of read errors. This leaves IC and RF microchip systems, both of which are available in readonly and read-write configurations.

In an FMS environment, a read-only system has several advantages over a readwrite system. With a read-write system, the amount of tooling data that can be stored is limited. Read-only storage is limited only by the capacity of the host computer data 


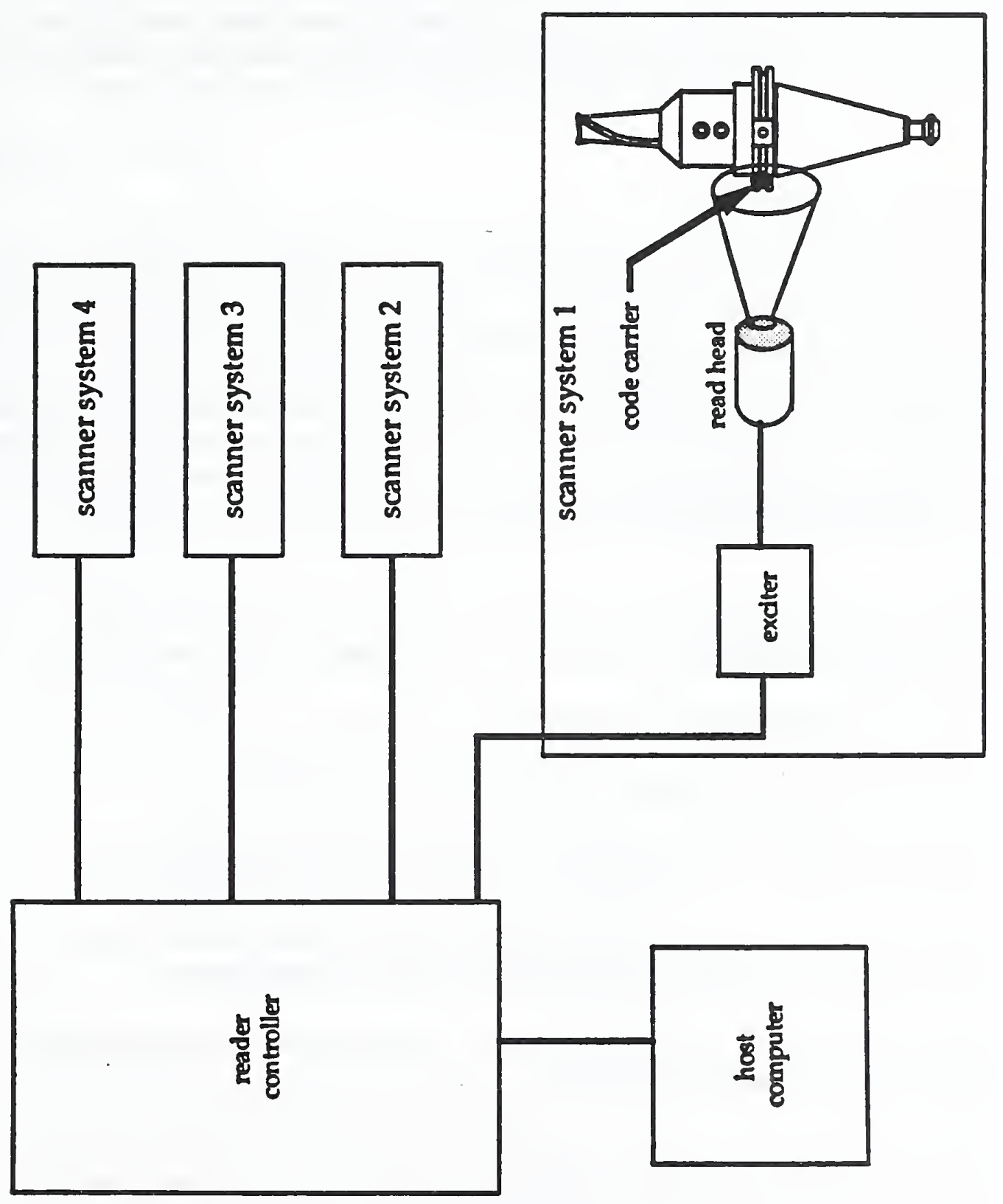

Figure 6-1. Tool Identification System 
base. In a read-write system, information can be accidentally or erroneously changed on the shop floor. In a read only system data is stored in computer data base, access to change this data can be restricted. Since read-write systems carry more data, read times are slower than with read-only systems. With read-write systems, tooling data is carried on the chip. Updating this data requires that the tool be stationary during the writing and verification cycle. This would add extra time to the machining cycle.

\subsection{Tool Assembly Tracking}

The TMS will provide a tool assembly data base, tool assembly identification system and interfaces to control systems to allow constant real-time tracking of the location and status of all the tool assemblies the manufacturing system. The tool tracking system will automatically update the tool assembly data base when the status (e.g., remaining life) or location of a tool assembly changes. Tool identities will be read each time a tool assembly enters or leaves the tool room, enters or leaves a machining cell, enters or leaves a storage buffer, enters or leaves a machine tool magazine, passes a read head in a magazine, or is placed in a spindle.

\section{Functions}

* Post tool assembly location and status changes to data bases

* Locate tool assemblies in the system

Timing requirements for performing functions

* User data entry - see section 8.4 for user data entry requirements.

* Display terminal - Screens should be updated with selected report screen within 3 seconds of the user making a new selection.

* Tool assembly data base transactions - Data base transactions should take no longer than 30 seconds.

* Tool assembly data - Data acquisition from the tool assembly data base should be completed within 5 seconds after operator indicates the desired tool assembly. The system should be immediately ready to respond to the next operator request after completing data acquisition.

* Tool assembly status updates - updates to the tool assembly data base should be made every time a tool assembly is moved. 
* Tool assembly status updates from machine tools - updates to the tool assembly data base should be made immediately after every execution of an NC program (e.g., new remaining life and new location in the magazine). Machine tools will communicate with the cell controller which will update the local cell data base and then send an update to the tool data base over the network link.

\section{Hardware requirements}

* Communication (network) links to the tooling data base(s) including local cell data bases.

* Communication (network) links to the cell controllers

* Software required to access tooling data base(s) and interface with the tool identification system.

* One data entry and display terminal shall be provided local each machining cell and the tool room.

* The user interface provided on the terminals shall be support data entry and display operations for at least the following types of activities:

- View a list of all tool assemblies showing location, assigned job, remaining life

- View the location and status of a tool assembly

- View a Tool Setup Specification

- Change the status of a tool assembly

- Display a Tool Assembly Drawing

- Generate Tool Room Exception Reports

- Initiate the printing of hardcopy reports

* Data acquisition communications interfaces - Suitable hardware interfaces shall be provided for the purpose of communicating data from the tool identification system.

* Tool identification reader locations:

- Tool room - at each presetting station, issue area, receiving area

- Machine tools - at each machine tool 
- Machining cells - at the entry/exit point to each machining cell

- Buffer storage locations - at each buffer storage location inside each machining cell

* Tool transport device reader locations:

- ASRS entry/exit points

- Tool room - issue and receiving area

- Machining cells - at the tool transport device receiving area

System inputs

* Tool assembly data from tool setting stations (D,T)

* Operator display selections (T)

* Tool assembly status requests (T,S)

* Tool assembly status updates (T)

System outputs

* Tool assembly data (T,S,D,P)

* Tool assembly status and location (T,S,D,P)

* Data acquisition status indication (R,T)

Discussion

The location and status of all tool assemblies in the manufacturing system must be constantly tracked and kept up-to-date. Tool assembly status and location information must reside in a central data base that is accessible to other manufacturing disciplines such as; process planning, scheduling, etc. Tool assembly status information may also reside in a local cell controller data base to reduce access time and central data base transactions. For example, the K\&T cell controller would probably maintain its own local tooling data base to reduce access time to tool assembly data. But, tooling data must not reside solely at the cell controllers. All tooling data must eventually be transmitted to the central tool management data base. Maintaining tool assembly status and location data is one of the most important tool management functions. 


\subsection{Tool Monitoring at Machine Tools}

Tool monitoring at machine tools has several important functions; to ensure that a tool never breaks during a cutting operation, to ensure that a broken tool is never used for a cutting operation, and to gather data on tooling performance for later processing by engineering staff.

\section{Functions}

* Measure tool length, diameter, and flank wear of each tool before it is used for machining operations.

* Check tool measurements against data base values (note: initial tool length and diameter values are measured in the tool room during presetting operations).

* Monitor spindle torque and axes thrust and compare these values to specified limits.

Timing requirements for performing functions

* Probe measurements - Probe measurements should take no more than 5 seconds.

* Torque and thrust monitors - Torque and thrust data should be available instantaneously during machining operations.

* Torque and thrust limits - The spindle should retract immediately when specified torque or thrust limits are reached.

\section{Hardware requirements}

* Provide a means to measure tool length, diameter, and flank wear at each machining center. (Note: length and diameter are general terms, tool wear is usually judged by the amount of flank wear, probes could be positioned to measuring material loss at many positions on the tool edge depending on cutter type, etc.). The refined tool length and diameter values would be used as a form of "adaptive" control for real-time corrective action. 
* Provide a means to check tool measurements against data base values (note: initial tool length and diameter values are measured in the tool room during presetting operations). Differences between current and previous (data base) values indicate tool wear.

* Provide a means to monitor spindle torque and axes thrust and comparing these values to specified limits.

* Tool probes permanently mounted at machine tools, tied in to machine tool controllers.

* Probes should be capable of measuring tool length and diameter to 0.0001 inch.

* Software for communicating probe measurements to the cell controller data base (note: everything sent to a cell controller data base is subsequently copied to the central tool data base).

* The machine tool controller must generate a probe error message when a probe error occurs.

* The operator should always have the option of deciding that a tool is behaving irregularly. The operator should be able to identify the tool as a worn tool by entering commands into a terminal.

* The system must not be affected by extraneous radio frequency energy or electrical noise.

System inputs

* Message receipt acknowledgments (S,D)

System outputs

* Probe measurements (E,S,D)

* Read error messages (E,S,D)

* Torque and thrust readings (S,D)

Discussion

A cutting tool is considered worn when it is no longer capable of holding geometric tolerances and finishes. Since the individual machining centers in an FMC are 
not operated on a one man for one machine basis, it is essential to monitor cutters for wear and breakage, and to keep a record of remaining tool-life. Most FMC control systems rely on predetermined cutter lives, based on past data, to replace worn tools.

The FMC controller monitors elapsed cutting time for each tool. When the preset value has ben reached, a tool change is performed. One disadvantage is that predetermined cutting life values are usually conservative, resulting in underutilized tools. This leads to increased tooling costs and additional time spent performing tool changes. Also, broken tools go undetected.

A preferable method for detecting cutter wear or breakage is by using a fixed probe permanently mounted on the machine tool. The NC part programs contain routines that check the cutter for wear. The cutting tool in the machine spindle is brought into contact with a fixed probe. The probe software verifies tool length (another probe could be used for diameter), which is specified with a tolerance in the tool assembly data base. Any change in the tool length exceeding tolerances indicates either a worn or broken tool. Tool offsets can be updated as the tool wears.

Several other methods have been developed to monitor tool wear or breakage. These include:

* Cutting force - As a cutter begins to dull, feed force increases. Also, if a cutter breaks, there is a sudden decrease in feed force. The cutting force components (\% of max load) and the flank wear are measured and plotted for any correlation. Once a complete correlation has been established, the magnitude of the cutting force, measured while cutting, is used to predict tool wear and remaining tool-life without interrupting machining. The Freeze Frame system already collects in-process cutting force data. With the proper software, Freeze Frame results could be used to constantly modify remaining tool-life estimates.

* Torque and power - The amount of power drawn by the machine spindle is monitored. A dull tool requires more power than a sharp tool. Control limits are set based on predetermined torque and power values for maximum acceptable tool wear. Also, a correlation between tool wear and torque-power readings is established. For this system to be successful, very sensitive torque sensing instruments must be used. This system should be used in conjunction with contact sensing. 
* Vibration - As a tool dulls, its cutting edge rubs against the work piece a produces different frequency vibrations. Vibration frequency patterns are correlated against tool wear. High vibration frequencies indicate imminent tool failure.

* Cutting temperature - The temperature at the tool-work piece interface is measured by infrared radiation or by thermocouples. After the establishment of a correlation, the temperature being measured is used for on-line prediction of tool wear.

* Radioactive sensing - A small amount of radioactive material is implanted in the tool flank. At the end of each cycle the amount of radioactivity is measured to determine the amount of flank wear (One can imagine the OSHA and disposal problems with radioactive tools).

* Electrical resistance - Flank wear is measured by sensing the electric resistance of a thin film coated on the tool flank face.

* Acoustic frequencies - A sensor measures the amount of ultrasonic energy transmitted from the cutter. The acoustic frequency patterns of the cutter are correlated to tool wear.

* Vision - The flank wear is measured directly using an ITV camera.

* Optical - The edge of a cutter is illuminated, and the vision system analyzes the reflected pattern. Any changes indicate tool wear or breakage.

All these sensing and monitoring schemes require the input of predetermined data corresponding to cutter wear and breakage. If the measured values signify excessive wear, the tool is replaced. If the measured value indicates a possible broken cutter, the machine is halted and an error message alerts the operator. Except for the first two, the above sensing methods all suffer from various implementation problems - the least of which is that they are not commercially available. Some require sophisticated instrumentation, most work well in the laboratory, but are unproven in a production environment. Contact sensing and torque/force monitoring are the current methods of choice with vision and optical methods as promising future choices. 


\subsection{Tool Data Collection at Machine Tools (Freeze Frame)}

One of the most important functions of tool monitoring is to collect and record cutting parameters during the machining cycle. This data is used by the engineering staff to predict tool-life, determine what factors influence tool wear, calculate optimum feeds and speeds, evaluate vendor performance, and evaluate special cutter coatings, cryogenic treatments, etc.

\section{Functions}

* Record cutting data at preprogrammed points during the machining cycle (Freeze Frame). This data should include:

- machine number

- tool number

- tool assembly number (microlog number)

- date/time (y,m,d,h,m,s)

- position $(x, y, z)$

- spindle RPM

- spindle horsepower

- feed rate

$-x, y, z$, axis thrust (as a \% of maximum)

- depth of cut

- feature number (e.g., hole number)

* Transmit collected data from machine tool controllers to cell controller data base (note: all data transmitted to the cell controller data base is subsequently copied to the central tool data base).

Timing requirements for performing functions

* Data collected during the execution of an NC program should be transmitted just after the program is completed - before the next NC program is started.

* Cell controller must immediately acknowledge receipt of data files.

* Timing requirements for Freeze Frame data acquisition software on machine tool controllers will not be discussed here (it already works). 
Hardware requirements

* Communications interface between individual machine tool controllers and cell controllers capable of transmitting data collected during the machining cycle.

System inputs

* Commands to initiate data collection (in NC programs) $(\mathrm{S}, \mathrm{T})$

* Data transfer receipt acknowledgment. $(S, D, T)$

System outputs

* Real-time cutter data $(D, P)$

Discussion

RIA staff developed a real-time cutter data collection system called "Freeze Frame" to evaluate tooling performance. This system has been used successfully, but the data collection is currently a manual operation. This should be upgraded to an automatic system. Cutter data should be transmitted to the tooling data base rather than printed at the machine tool. Cutter data could then be read from the data base, processed, and plotted for analysis. 
Tool Tracking and Data Collection

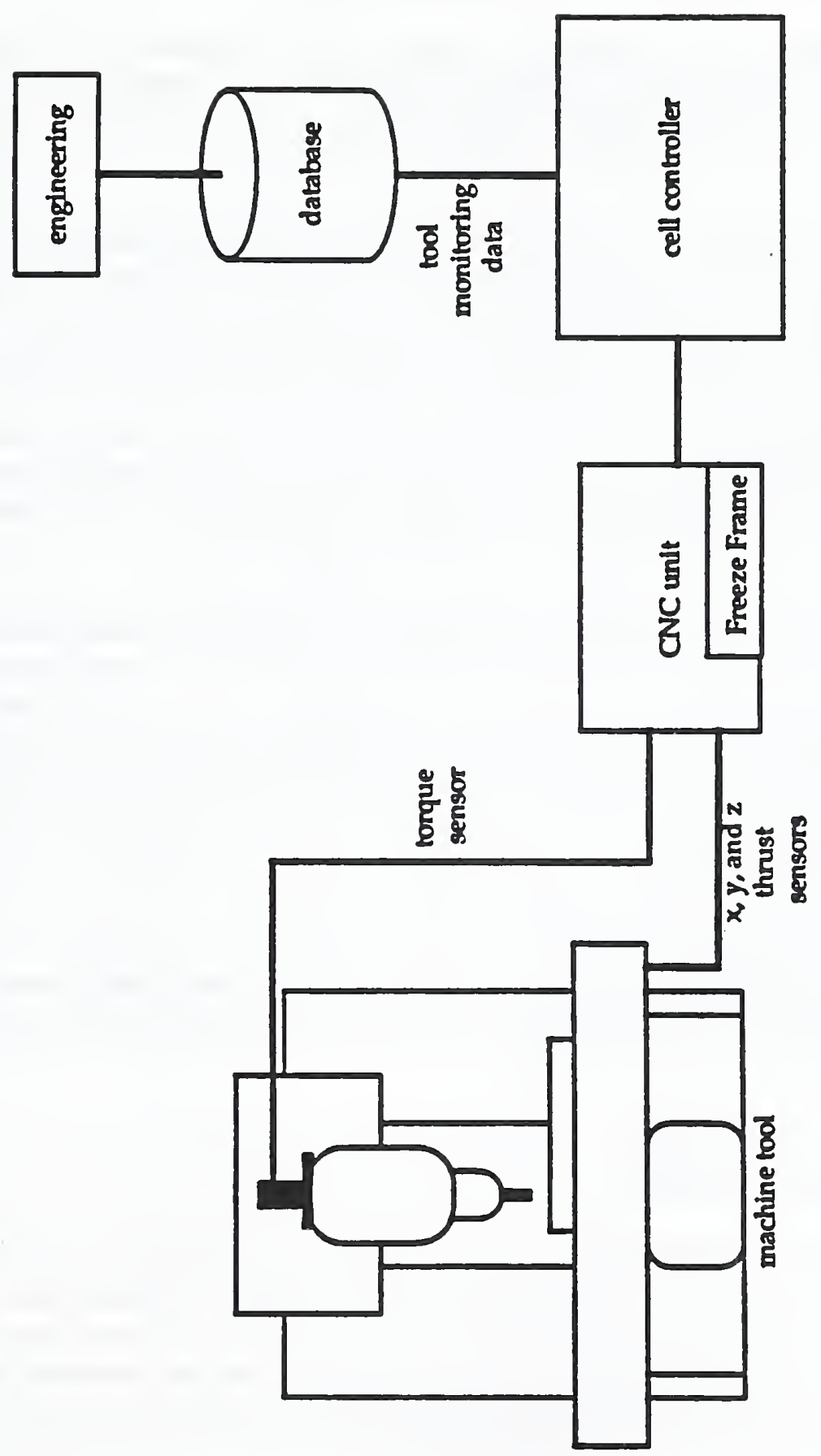

Figure 6-2. Freeze Frame real-time data collection system 


\section{Tool Management Upgrades to the Existing Cell}

The existing FMC currently consists of two Kearney and Trecker MM-800 horizontal machining centers, a common pallet shuttle car, 12 part load/unload stations, and a K\&T level I cell controller. The Flexcell is presently being upgraded to add additional machines and control capability.

When this upgrade is completed, the cell will consist of two three K\&T MM-800 horizontal machining centers, a third horizontal machining center, one 4-axis coordinate measurement machine, four K\&T Orion-2300 machining centers, a common pallet shuttle car, 52 part queue stations, a tool delivery robot, tool ID system, and an upgraded cell controller. In addition, all seven machine tool magazines will be upgraded to incorporate tool changer arms and small tool buffers to facilitate tool exchange with the tool delivery robot. Added control capabilities will include in-process tool monitoring (Freeze Frame; already in use), tool wear sensing, machine tool monitoring, tool assembly tracking, network communications, and database interaction.

The cell controller's LAN connection will enable it to communicate with the various manufacturing databases and other elements in the manufacturing system. This network connection will permit the cell controller to obtain tool assembly data (e.g., offsets, remaining life, etc.), receive NC part programs, and store data for other functions such as statistical process control. This increased access to manufacturing data, combined with planned sensory enhancements will allow the cell controller to perform advanced tool management functions.

The tool management upgrades will provide the capability to migrate tooling within the cell and optimize tool usage. The tool identifications system will permit tool assembly tracking. Tool monitoring and wear sensing systems will provide the information required to identify dull tools.

\subsection{Tool Handling by Robot(s)}

The robotic tool delivery system shall be responsible for transferring tool assemblies between pickup/delivery points, tool buffer storage areas, reader stations, and machine tool magazines within the cell. The tool buffer storage areas store tool assemblies that have been delivered to the cell but are not yet needed in individual machine tool magazines. Due to space availability considerations, the robot should be configured as a mobile overhead system. 


\section{Functions}

* Move tool assemblies between ready stations within the cell

* Exchange tools between grippers and ready stations

* Move tool assemblies to/from buffer storage areas

* Move tool assemblies to/from cell pickup/delivery point

* Position tool assemblies at read stations

* Perform self-calibration checks

* Provide system status to supervisory system

Timing requirements for performing functions

* Tool moves - A robot tool transfer between any of two of the stations listed above should complete in less than 90 seconds. The robot should be capable of completing this task from an idle state anywhere in the system. It should be capable of performing all communications, sensor readings and safety checks necessary to perform this function in the allotted 90 seconds. Tools are assumed to be in a ready position at the beginning of the operation.

* Tool exchanges - An exchange between the magazine and the ready for pickup/drop-off position should be completed in 30 seconds or less.

* Tool identification reads - When positioned at the read station, a robot should complete a tool identification read sequence in less than 10 seconds.

* Acknowledgement of commands - The robot controller should issue a response message or status report to supervisory controller within 15 seconds of receipt.

* Power-up self checks - When the robot is brought up from a cold start or after a loss of power, it should perform all internal diagnostic checks and axes calibrations within 5 minutes.

\section{Hardware requirements}

* The tool delivery robot system must be capable of delivering tools to and removing tools from the following equipment: the three K\&T MM-800 machining centers, the four K\&T Orion-2300 machining centers, and the tool buffer storage areas within the cell.

* The robot should be completely electrically operated. 
* All axes should be powered by AC servo motors with position feedback.

* The robot should be equipped with two electrically operated grippers capable of handling 50 taper, Cat standard tooling. In the case of a monorail robot system, a single gripper on each robot may be sufficient.

* The robot should be capable of carrying two tools at the same time, each weighing as much as 60 pounds. In the case of a monorail robot system, handling a single tool at a time may be sufficient.

* The robot should be capable of the following minimum travel speeds:

- Longitudinal axis (length of cell)

- Vertical axis

- Beam axis (width of cell)

- Grippers

- Gripper rotation
$40 \mathrm{in} / \mathrm{s}$

$20 \mathrm{in} / \mathrm{s}$

$20 \mathrm{in} / \mathrm{s}$ (Gantry robot only)

$12 \mathrm{in} / \mathrm{s}$

$60 \mathrm{deg} / \mathrm{s}$

* The robot delivery system should operate efficiently without excessive wear to tooling and tool handling components. The robot should be capable of the following minimum positioning and repeatability accuracies:

- Linear axes

- Gripper rotation \pm 0.025 inch

\pm 1.0 degree

* The robot delivery system should be equipped with an incoming/outgoing tool assembly transporter receiving area at the north end of the cell. The tool transport device should be carried by a fork truck (or AGV) to and from the tool set area. The tool assembly transport device must be provided with sufficient locators at the delivery robot site to ensure positive and continued orientation in relation to the robot axes. The transport device should be able to carry a minimum of 24 tool assemblies.

* A stationary read unit should be provided at the transporter receiving area to identify tool assembly transporters. The same identification system should be used as is used for the tooling.

* Sufficient sensors must be provided to determine if a tool transporter is in place and secured. 
* The identity of incoming/outgoing tooling should be verified as it is being handled by the robot. A read head mounted on the robot gripper should be considered.

* The tool delivery robot should be programmed for specific paths between machine tools and storage buffers.

* The robot controller shall be configured so as to support a number of different communication options with the supervisory controller. A variety of communications capabilities shall be offered as alternative options: direct RS-232, Ethernet, Map network, etc.

* The robot control system shall have a terminal and a teach pendant for operating in stand alone mode.

* Sufficient calibration points and mechanisms should be provided so that robot can automatically self-calibrate.

System inputs

* Move, tool exchange, calibrate commands $(S, T)$

* Status requests $(\mathrm{S}, \mathrm{T})$

System outputs

* Robot physical movement and handling operations (E)

* Command feedback (S,T)

* Status reports $(\mathrm{S}, \mathrm{T})$

* Error Messages (S,T)

Discussion

The requirements for the robot delivery system and the tool buffer stations at the cell have not yet been completely specified. Several alternatives are presently being considered. The most recently considered option consists of horizontally-mounted chain-driven magazine(s) and a continuous track monorail robot system. In a completely automated system, a stationary robot (mechanism) would be used to transfer tool assemblies between an AGV delivered tool transport device and the horizontal tool carousel. In a manual tool delivery mode, the tool assemblies would be loaded into the system by hand. A tool identification reader mounted on the carousel would continuously verify the identities of tool assemblies placed in the carousel. 

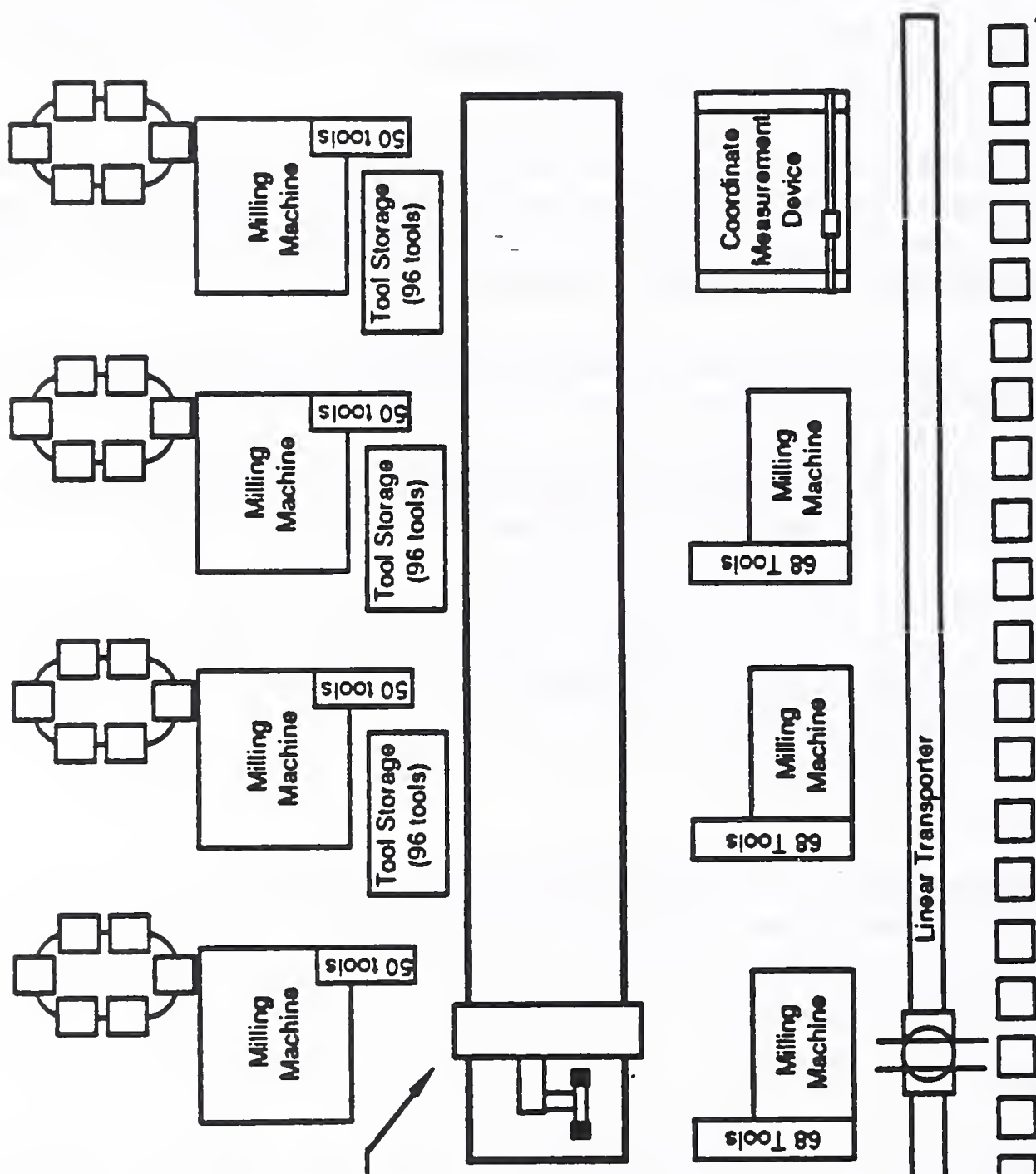

施

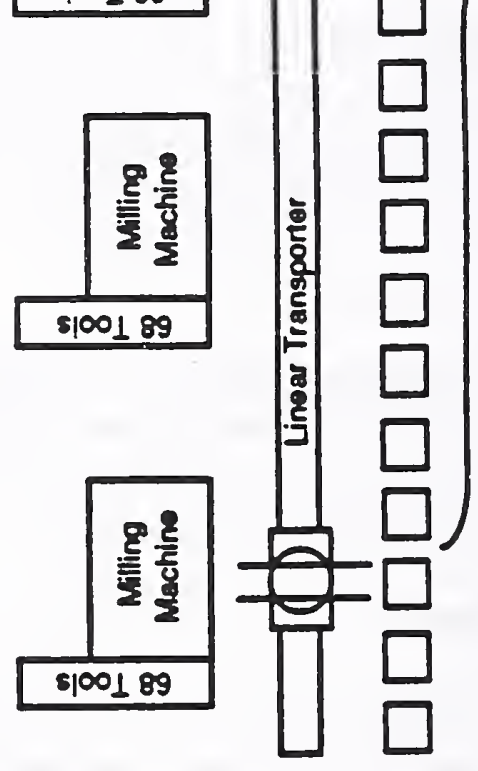

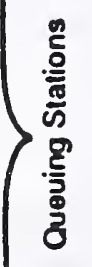

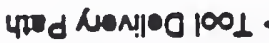

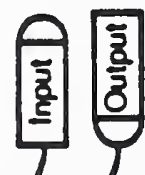

을

Figure 7-1. Gantry robot configured with tool buffers racks 
Tool Management Upgrades to the Existing Cell

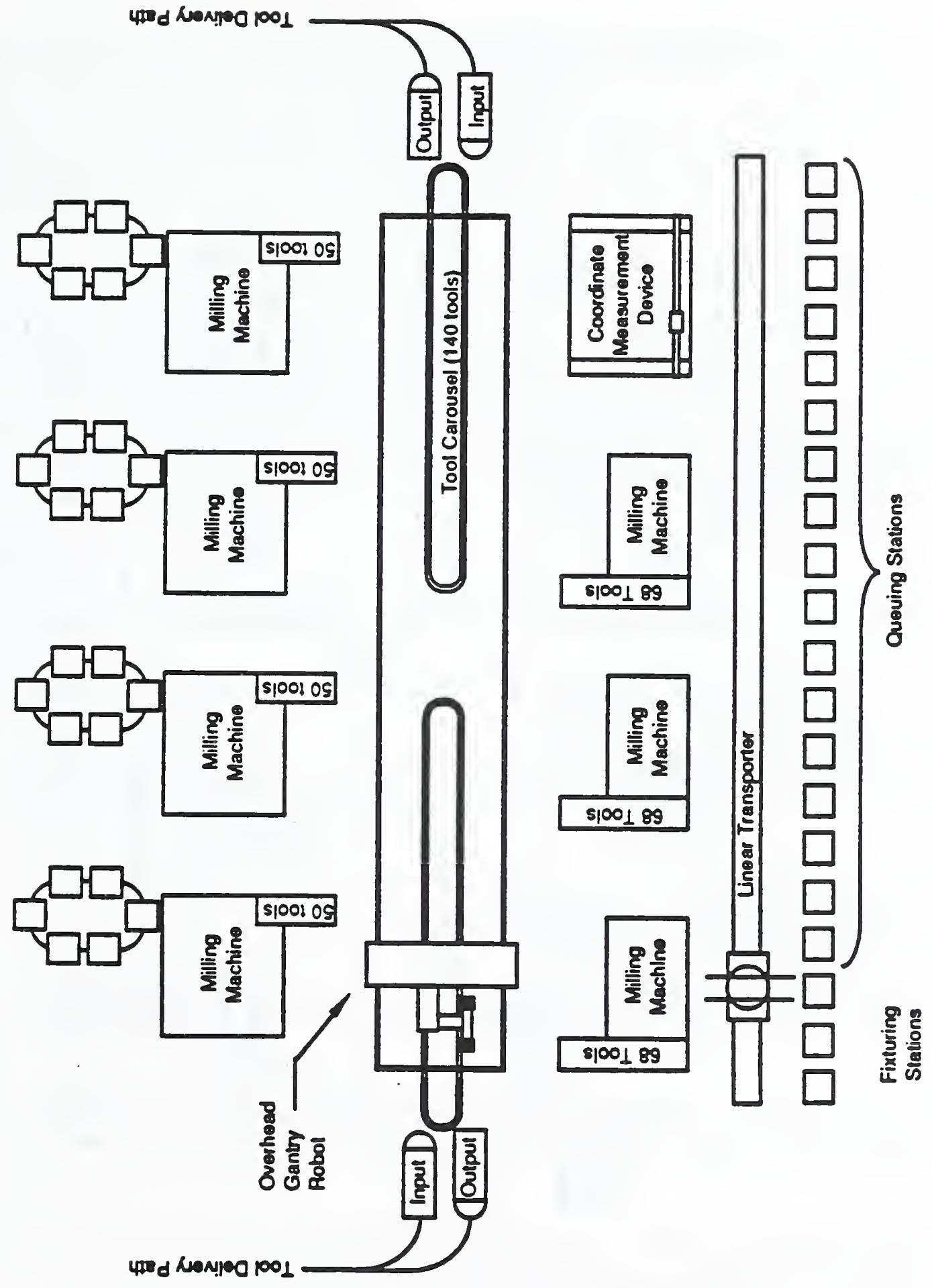

Figure 7-2. Gantry robot configured with carousel tool storage 


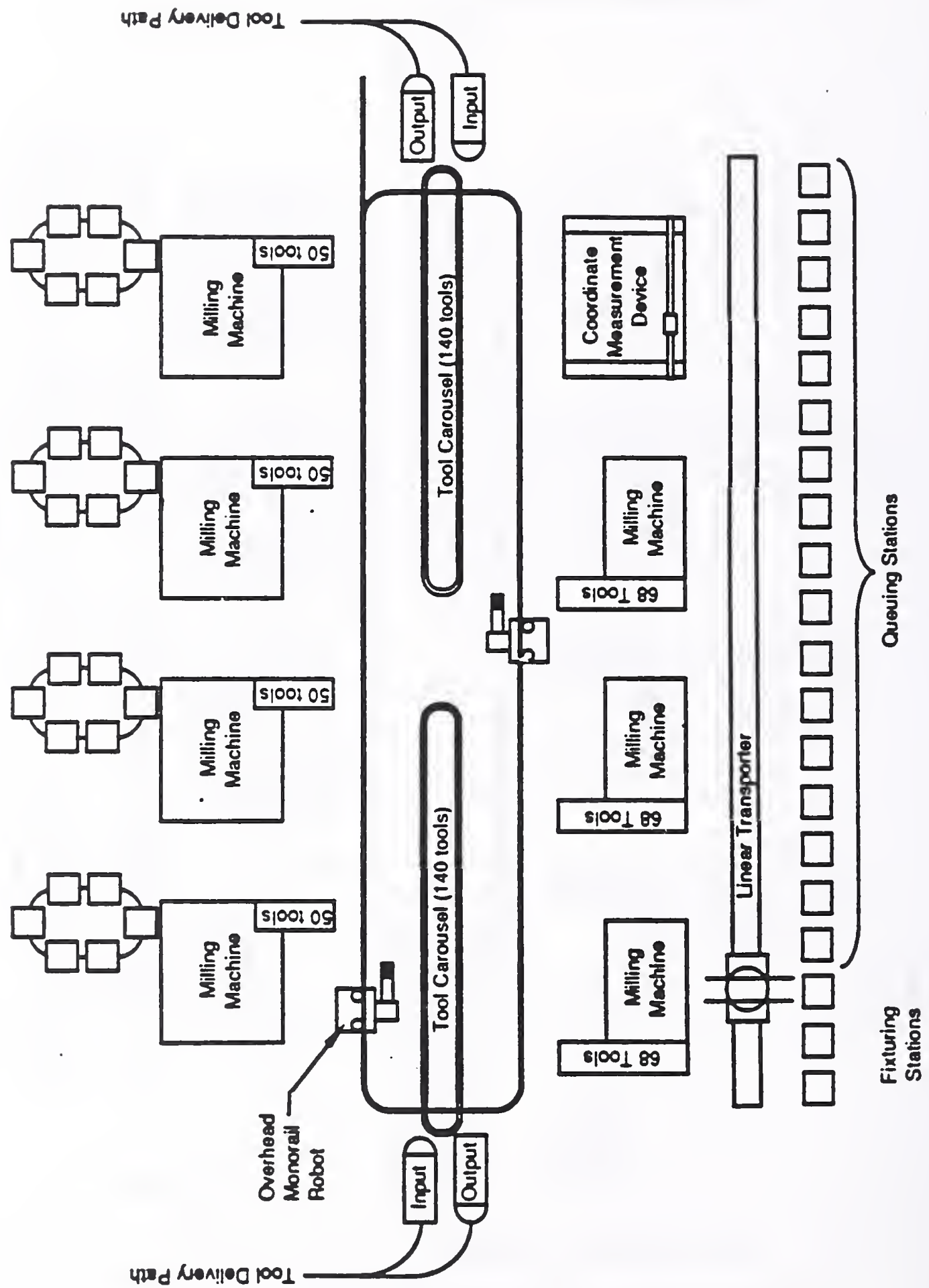

Figure 7-3. Monorail robot configured with carousel tool storage 
A monorail robot system has an advantage over a gantry type robot system. In a monorail system, two robots would operate on a continuous loop track. One would predominantly service the four Orion-2300 machining centers and the other would service the three MM-800's. Both would be capable of servicing all seven machine tools. The tool buffer storage areas would be mutually accessible. In the case of a failure, tool handling operations could continue with one robot while the other was serviced on a small side track (see Figure 7-1, 7-2, and 7-3).

\subsection{Loading and Unloading of Tool Magazines}

Tool assemblies must be transferred between the machine tool ready stations (robot pickup and delivery points at the machine tools) and the machine tool magazines. This transfer will be accomplished with a tool changer arm controlled by the machine tool controller. This system should also include a reader to positively identify tools during each transfer operation.

\section{Functions}

* Move tool assemblies between ready stations and machine tool magazines Timing requirements for performing functions

* Tool exchanges - An exchange between a machine tool magazine and the ready for pickup/drop-off position should be completed in 30 seconds or less, including magazine search time.

* Tool identification reads - When positioned at the ready station, a tool identification read sequence should be completed in less than 10 seconds.

* Acknowledgement of command - The machine tool controller should issue a command received response message to the cell controller within 5 seconds of receipt.

* Transfer completed message - The machine tool controller should issue a transfer completed message to the cell controller within 5 seconds after a transfer is finished.

* Spindle/robot tool exchange coordination - Tool exchanges at the machine tool storage magazine shall not take place unless the machining center has at least one minute of cutting time remaining with the current tool in the spindle. During a tool exchange between the magazine and the 
ready station, the machine tool controller will not attempt to perform a tool exchange at the spindle until the ready station exchange is completed. The machine tool magazine shall be returned to NC program control as soon as the tool enters/clears the socket.

\section{Hardware requirements}

* Each machining center shall be equipped with a tool transfer device capable of removing a tool from the machine tool storage magazine, placing the tool on a transfer station for pick up by the robot, picking up a tool deposited on the transfer station by the robot and placing the tool in the machine tool storage magazine.

* The system should be capable of exchanging tools weighing as much as 60 pounds.

* The identity of a tool being exchanged shall be verified by a stationary read head at the ready station as it leaves/enters the machine tool magazine. Sufficient sensors must be provided to determine if a tool assembly is present at the ready station.

* It shall be possible to operate the exchanger arm manually (MDI).

System inputs

* Exchange tool commands (S,T)

System outputs

* Command feedback (S,T)

* Error Messages (S,T)

\section{Discussion}

The differences between the tool storage magazines on the MM-800's and the Orion-2300's have caused several problems in designing a suitable robot - magazine interface. The tool assemblies on the MM-800's are held level and secured by a clip. Tool assemblies on the Orion-2300's are held at 45 degree angle and are not secured by a clip. Excessive force is required to extract the tool assemblies from the MM-800's. A neutral interface mechanism solves this problem at the expense of adding more moving parts to the system. 
This expense is justified because the neutral interface also solves the problem of coordinating tool exchanges at machine tool spindles with robot movements. Having the robot wait at a machine tool while a tool assembly is brought into position or having a machine tool idle while the robot loads a tool places severe overhead on the system. With the ready stations and exchange mechanisms the robot can perform pick-ups and deliveries independent of machine tool storage magazine movement.

The neutral interface also provides the option of replacing any of the machining centers with no effect on the robot delivery system. If a machine is replaced with a machine from a different supplier, only a customized exchange mechanism is needed to integrate the new machine into the cell (plus a control interface with the cell controller).

\subsection{Tool Storage in Buffer Areas}

A considerable number of tools will need to be positioned in the machining cell to avoid delaying production jobs. These buffer storage areas will have to be readily accessible to the overhead robot that will deliver the tools to the exchange points behind each machine tool magazine. The storage areas must be configured so that the robot has clear access to each tool storage position and large tools do not interfere with each other.

\section{Functions}

* Provide storage locations for tool assemblies within the machining cell.

Timing requirements for performing functions - N/A

\section{Hardware requirements}

* Storage devices should be capable of handling tools assemblies that weigh up to 60 pounds. The system should store tool assemblies with diameters up to 4.5 inches with adjacent pockets full and tool assemblies with diameters up to 9.0 inches with adjacent pockets empty.

* A buffer storage device should be equipped with sensors to detect the presence of a tool assembly in any of its storage locations.

* Tool identification readers should be located at all tool storage buffers.

* All storage positions within a storage area should be easily accessible by shop personnel. 


\section{System inputs}

* Tool assemblies (E)

System outputs

* Tool assemblies (E)

Discussion

See the discussion in Section 7.1.

\subsection{Cell Controller}

The cell controller is responsible for distributing NC programs and tooling data to the machine tools within the machining cell. It also directs the movements of the pallet exchange mechanism and the tool delivery robot.

Functions

* Delivery robot sequencing.

* Perform tool allocation based on tool allocation strategies defined by the user.

* Manage tool monitoring data.

* Access manufacturing databases to perform the following functions:

- Obtain tooling data

- Obtain NC part programs

- Store tool monitoring data

- Store tool assembly status data

* Transmit NC part programs and tooling data to machine tools within the cell.

* Identify worn tools and initiate tool exchange.

* Provide tool location data to the tool management system. 
Timing requirements for performing functions

* Tool assembly data - Tool assembly data (e.g. tool offsets) should be obtained from the tool management database immediately after the tool assembly enters the machining cell.

* Worn tool replacement requests - Worn tool replacement requests should be posted when a tool assembly reaches $75 \%$ of its projected life.

* Tool location data reports - The system must respond to requests from the TMS controller to provide data reports which identify the location and status data for all tools within the cell within 60 seconds.

Hardware requirements

* Network communications interface to LAN (to be determined).

* Sufficient memory capacity to store a minimum of 1000 part programs, 1000 tool assembly data files, tool buffer storage location data, etc. (approximately 256 megabytes of disk storage).

System inputs

* Part production schedules (D,T)

* NC part programs (D)

* Tool assembly data (D)

System outputs

* Tool monitoring data $(\mathrm{D}, \mathrm{T}, \mathrm{P})$

* Tool assembly status and location within the cell $(D, T, P)$

Discussion

Due to the existing configuration of machining centers within the cell, it is likely that this function will be performed by an upgraded cell controller. It is an off-the-shelf system that will require some custom upgrades to satisfy these requirements. The upgrades will be principally to provide access to data structures that are maintained in internal proprietary formats. 


\section{Information Services}

This function is a composite of three major areas of information systems requirements that form the glue that holds the entire network of tool management systems together. Data base management acts as a repository for all information that is shared by more than one subsystem (See Figure 8-1). The communications network connects that various computer system by passing messages between various application programs. It also connects applications with the data base management system and peripheral devices, e.g., terminals and printers. The peripheral devices are primarily terminals or work stations where users can interact with various tool management subsystems.

\subsection{Data base Management}

The data base management system is a critical component of the tool management system. The data base is used to store the lasting information needed by any of the tool management functions. The system is composed of a data dictionary, a core commercial data base management systems (DBMS), physical data storage devices, logical view processors (report generators), data manipulation language (DML) translators, and supervisory software. Some examples of the types of information that will be maintained in the tool management data bases include:

- Tool setup specifications

- Tool drawings

- Tool assembly location

- Component inventory

- Tool Performance

The operation of the data administration system should be transparent to the systems that it supports. Computer processes within the tool management system post data base queries and update transactions via messages passed over the communications network. All transactions are written in a neutral, non-proprietary DML syntax. Data administration system servers translate the transaction into the proprietary language of the appropriate commercial DBMS to access or update the data bases. This neutral DML approach ensures that applications developers can construct systems that are dependent upon specific DBMS implementations.

Data base Command/Status Messages - A protocol is required by which application processes interact with the data administration system and the common data bases. ANSI/ISO Abstract Syntax Notation (ASN.1) standards can be used as the specification language and encoding syntax for specifying the format of command and 
Information Services

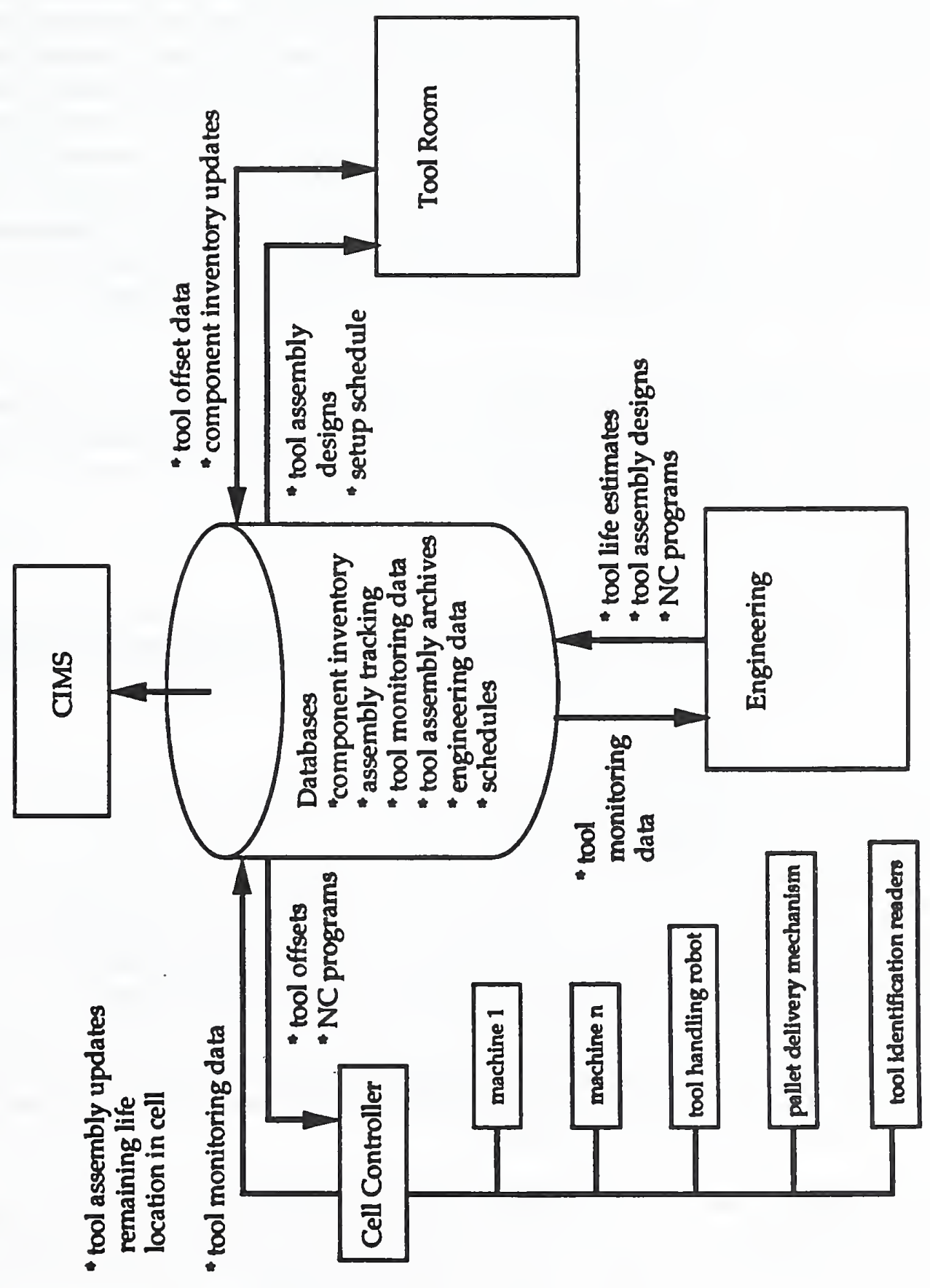

Figure 8-1. Tool management data bases 
status messages to the data base system. The protocol for interacting with the data base should support the grouping of multiple transactions, which may be targeted at physically distributed data bases, in a single data base transaction message. Command message formats need to be defined which may be used to initialize, start up, terminate and abort data base interactions. Data base queries, which contain Data Manipulation Language (DML) statements, are also specified within the format of command messages. Fields of data base command messages should include a message number, a user identifier, and a command in one of the formats listed above. A data base query type command is simply a DML statement. The DML syntax used is a subset of the ANSI Relational Data base Structured Query Language (SQL) Specification. Status messages describe the state of the link to the data base and identify the commands and queries to which the data administration system is currently responding.

Data base Reports - The data base command/status messages, outlined above, should trigger the posting and/or retrieval of data base reports. These reports should be globally defined, logical groupings of information which are exchanged between applications systems and the data base. Reports will need to be defined for all applications within the tool management system.

\section{Functions}

* The system will handle all routine data base transactions supported by the SQL (Structured Query Language) specification, including:

- Insertion

- Retrieval

- Selection

- Deletion

* The system itself will be implemented as a relational data base - a technology in wide use in industries today. This approach will be taken since the primary role of the $d^{2}{ }^{t} \mathrm{n}$ base server is to provide reliable service.

* The data base will normally be operating as a background system, with an interface to all calling subsystems. It will operate purely as a server module, with the subsystems being clients.

* There will also be a simple dialogue interface for a user to directly examine the data base.

* The system will provide a full set of maintenance and support functions, such as transaction logging, backup and recovery tools. 
Timing requirements for performing functions

* Most interactions with the data bases will be from embedded programs, not from interactive sessions with a user generating SQL queries. Thus, the data base architecture must support program access, and should have a rapid response, typical queries should be processed in less than 30 seconds.

Hardware Requirements

* At least 512 megabytes of disk storage.

* Mini- or Microcomputer system with at least 10 megabytes of RAM memory to act as data base server.

System inputs

* Operator generated queries.

* Program generated queries.

System outputs

* Query responses

* Query status messages

Discussion

The RIA data base management system should be based on existing, proven, commercially available technology. RIA should not become a laboratory for testing new architectures or systems. Rather, robust and hardened systems should be combined to deal with the unique needs of RIA. In light of this, a relational data base approach would be most appropriate. Relational systems have been widely implemented in a variety of areas with proven success. They already support concurrency control (simultaneous multiple access) and transaction management issues. While some data base needs might be better handled with other approaches in the future, such as objectoriented data bases, needs such as concurrency control are only minimally addressed by these newer data base architectures. At this point the prudent course would be to use a relational system.

A question which invariably arises when integrating a new information system into an existing environment is how best to access existing data. One alternative is to migrate all existing data to the new data base system. However if there is a large body of data - and possibly several different data bases - this approach often becomes prohibitively expensive and time consuming. A more attractive alternative involves 
integrating the existing data bases with the new data bases into a single distributed data base architecture. Such distributed systems are commercially available, and generally rely upon the Structured Query Language (SQL) as the common query language shared by all data bases within the system. It would seem that this type of distributed data base architecture would be the best alternative for RIA. It will allow continued access to current data bases, since the data would not be moved. In addition, it allows the existing data bases to be accessible by new systems as they come on-line. Some code development may be required to provide the interface between the existing data base and the distributed system. The use of SQL as the query language is important since numerous commercial products are being released which are compatible with SQL.

By building the subsystems around a commercial DBMS, it becomes much easier to maintain information consistency. This leads to a cleaner set of data representations to be shared among subsystems as well as a clearly defined communication path between the systems. A single data base server obviates the need to construct custom interfaces between component systems and speeds performance. A single interface specification will be used between the data base and all client applications.

The data bases should be accessible through all the terminals making up the tool management system. Most, if not all, of the system functions rely on embedded data base transactions to provide or retrieve the supporting information. This means that there will be software running which will insulate a user from actually composing data base requests manually. In terms of connections, however, all terminals should have the same configuration.

\subsection{Tool Management Data bases}

This section identifies some of the data bases that should be maintained by the data base management system to support tool management.

\section{Functions}

* The tool management data base management system should be able to support at least the following functions:

- Tool inventory

- Requisition reporting

- Requisition document preparation

- Receiving information

- Obsolete tool reporting

- Expendable tool reporting 
- Tool equivalence reporting

- Cycle counting

- Tool-life and tracking

- Tool allocation and scheduling

- Tool preparation

- Tool specification (assembly definition)

- Historical data - maintenance and usage records

- Tool requirements projection

- "Where used" reporting

- Multiple tool dispositions - available, rework, in-use.

- Reporting based on center, part, order, job or location.

- Tool-kit maintenance

- Multi-level kitting

- Time-phased allocations

- Tool catalogs

Timing requirements for performing functions - N/A

Hardware Requirements - N/A

System inputs.

* Operator generated queries $(T)$

* Programmatic queries $(S, E, R)$

System outputs

* Reports and status messages ( $T, S, E, R)$

Discussion

The data base should reflect a classification taxonomy of tools, as well as tool assemblies. An appropriate top-level classification is:

* Tool

* Processing tool

* Locating tool

* Tool assembly

* Tool holder 
Attributes or fields which should be present include:

* Status - whether a given tool is available, or assigned to a job Tool id - used to uniquely identify a tool or class of tools. Tool bar code (if used)

* Tool manufacturer and/or vendor

* Reorder point

* Tool cost

* Remaining tool-life

* Expected tool-life

* Set length

* Tool assembly identification

* Cutter length

* Effective length

* Cutter angle

* Cutter radius

* Cutter number of flutes

* Extension diameter

* Extension length

* Extension type

* Adapter diameter

Additional attributes should be included as needed.

A client system must be able to generate arbitrary queries involving any combination of the above attributes and tool classes. This allows future expansion into unanticipated applications without requiring the rewriting of hard-coded access functions.

\subsection{Communications Network}

The transmission of messages between different software processes and computer hardware is the primary function of the communications network. The system includes the transmission media, network interface units on individual computers, and software to process messages for transmission. Processes should communicate with each other by writing and reading messages in memory areas that are accessible by both the process and the communications system. These shared memory areas are called mailboxes. The communications system is responsible for delivering messages from the source mailboxes written by applications processes to any destination mailboxes that are logically connected to them. This mailbox scheme keeps the inner-workings of the network transparent to the applications processes. 


\section{Functions}

* Transfer messages between mailboxes

* Maintain network connection data

* Handle recovery and retransmissions

Timing requirements for performing functions

* The network should support transmission rates commonly found in available local area networks.

* A distributed system requires a method of transferring information which is fast, accurate, reliable, and independent of the actual physical location of the machines. The transfer of information must also be done without interfering with or adversely affecting real-time processes on the receiving machine. This can be achieved either by direct originator-to-recipient message passing or by using a common (shared) memory.

\section{Hardware Requirements}

* The demands of the tool management system are likely to expand as new applications are identified at RIA. For this reason, it is important to implement a communications solution at this time which will have the capacity and flexibility to accommodate future needs. An appropriate solution is the implementation of a local area network based on Ethernet hardware. This system of cables and transceivers supports a number of networking protocols, any of which would be acceptable for tool management purposes.

System inputs

* Communications messages ( $\mathrm{S}, \mathrm{E}, \mathrm{T}, \mathrm{D}, \mathrm{R}, \mathrm{P})$

* Network management operations (T)

System outputs

* Communications messages $(\mathrm{S}, \mathrm{E}, \mathrm{T}, \mathrm{D}, \mathrm{R}, \mathrm{P})$

* Network status reports (T,D,P)

\section{Discussion}

In the area of factory communications, there have been a number of attempts made at industry-wide standardization. Any of several network protocols could be used 
with this local net implementation, including TCP/IP or MAP/TOP. Whatever specific protocol is chosen, it is well advised, but not mandatory, to use the same protocol for all systems on the network. Thus, communications with the data base system would use the same protocol as the rest of the tool management system. If different protocols are used for various parts of the system, gateways should be included to pass information from one protocol to another. While several local networks may be desirable, the use of different protocols is not recommended.

One of the key elements of the NIST AMRF communications network is the communications mailbox protocol. The structure of a mailbox includes a sequence number, a mailgram length, and a mailgram text area. The sequence number is used as a flag to indicate that the contents of the mailbox has been changed. The mailgram length reflects the total length of the current message contained within the text area of the mailbox. Mailboxes may be written by only one process, but may be read by many. Hence, a mailbox for incoming messages is typically written only by the network, while outgoing mailboxes are written only by one control process. A default set of input and output communications mailboxes are created for an application process when it is initiated. Additional mailboxes may be opened afterwards by a series of interactions with the data administration system. The synchronization of mailbox read and write operations is necessary to avoid accessing partially updated data. The methods used to synchronize common memory accesses are hardware and operating system dependent and will not be presented here.

Network management is accomplished by a human operator using a locallydeveloped software program. Configuration instructions are submitted by the operator and conveyed to the respective network interface program residing on a specific remote host. These instruction are used to build, monitor and disassemble the global common memory. Specifically, they establish the networked mailbox-to-mailbox linkages between remote local common memories that together constitute the global common memory.

The network interface programs (NIPs) build a mail delivery table based on the instructions received from the network manager. The mail delivery tables contain information identifying local mailboxes that are to be linked using network services and the location of other local common memories to which copies of updated mailgrams should be sent or from which mailgrams will be received. If the contents of a mailbox listed in the NIP's mail delivery table are changed, the respective NIP sends a copy of the new mailgram across the network to the destinations associated with that mailbox. Likewise, when the NIP receives a mailgram, it deposits it into the mailbox identified in the data packet and in accordance with the authorization implied by the information in the mail delivery table. 
8.4 Peripheral Devices - Terminals, Printers, Readers, etc.

These devices, such as: computer terminals, graphics monitors, status display panels, printers, plotters, tape and disk drives, light pens, tablets, bar code readers, etc. (See also Section 6 Tool Identification). The input/output devices are integrated under software control to form functional work stations for performing functions associated with: operator control and system monitoring, order entry, management information, tool room operations, inventory management, performance data analysis, maintenance diagnostics, etc.

\section{Functions}

* Data entry and display terminals shall be provided local to the assembly and presetting area. The display shall have sufficient graphic resolution to display schedules as Gantt charts or network displays.

* The user interface provided on this terminal shall be support data entry and display operations for at least the following types of activities:

- View text reports and forms

- View graphics representations of tool drawings

- Initiate the printing of hard copy reports

* Hard copy reports that shall be printable on the control room printer shall include all reports accessible at the user interface.

* The user interface shall be easy to use. Control room personnel should be able to become qualified in its operation with one day of hands-on training.

* The user interface shall employ a password security mechanism which prevents unauthorized personnel from modifying data that is defined as protected.

Timing requirements for performing functions

* User data entry - Keystrokes should be immediately echoed to screen. The delay time to echo should be less than a second to create the effect of an instantaneous response.

* Display terminal - Screens should be updated with selected report screen within 5 seconds of the user making a new selection. 
* Printed reports - A report should start printing within 30 seconds of the completion of a user request at a terminal, assuming that other reports are not already queued for printing.

\section{Hardware requirements}

* Display and data entry terminals - The terminal and keyboard must be hardened for use in an industrial environment. The graphics resolution should be at least 640 horizontal by 480 vertical pixels. At least 16 colors should be available on the screen.

* Printer - One printer shall be provided for shared access by all user terminals within the control room area. The printer shall support both graphics and text. It shall have sufficient graphics resolution to display the tool assembly drawings contained within the Tool Setup Specification. It shall be capable of printing 10 pages per minute. The printer must be capable of quiet operation or be contained within a sound enclosure.

* Data acquisition interfaces - Suitable communications interfaces shall be provided for the purpose of acquiring data from the tool presetting equipment and the tool identification system.

System inputs

* Peripheral device data streams, i.e., text and graphics (T,R,E,D,S)

System outputs

* Peripheral device data streams, i.e., text and graphics (T,R,E,D,S)

Discussion

See also the workbench user interfaces discussion in Section 9.4. 


\section{CIM System Links}

The TMS is initially expected to be a stand alone system. An open architecture should make integration with CIMS simpler. However, future integration into CIMS should be anticipated. Close cooperation with CIMS planners will be required to ensure sufficient compatibility (e.g., data base structures, network communications) between the TMS and CIMS to allow for any future integration. Although direct connections between TMS and CIMS will not be critical, initially, the following areas should be studied closely:

* data services and external data bases

* the distributed numerical control system

* network bridge or gateway

* workbench user interfaces

The remainder of this section roughly identifies requirements for possible future TMS to CIMS links.

\subsection{Data Services and External Data bases}

Retrieving data from external data bases is highly desirable if TMS is going to be integrated into the CIMS system. Data location, message formats, data transfer rates, data base query languages and redundancy of data should be considered. Some of the external data bases or files that the TMS will need access to are:

* NC programs

* tool lists

* tool setup specifications

* tool assembly drawings

* process plans

* dispatch lists

* master production schedules

* invientory

Furthermore, the CIMS system will need access to data maintained within internal TMS data bases. Some examples of the types of data that CIMS may need access to include:

* tool component inventory

* tool performance data

* TMS schedules

* job status information 
* system configuration and availability status

* system maintenance requirements

Utilization of common data base query languages, data base management and data dictionary systems will facilitate systems integration and the sharing of data between the TMS and CIMS.

\subsection{Distributed Numerical Control (DNC) System}

The present configuration of the DNC system will not permit the transfer of tool management data. The DNC system will support only the upload and download of NC programs to designated systems. If tool management systems other than the cell controller or machining centers need access to the NC programs, it is not clear that they will be able to access those programs. A link between the DNC system and the local TMS data base should be considered, if there is any possibility that access will be required by tool management systems or tool management personnel.

\subsection{Network Bridge or Gateway}

The TMS systems will be connected on their own local area network (LAN). The LAN should have sufficient capacity to support future expansion, including both additional TMS systems and bridges, or gateways, to permit communications between TMS and any CIMS system. When a LAN is selected for TMS, the availability of suitable bridges or gateways should be verified. The use of common basic message formats in both systems should also be investigated as it will help minimize integration problems. The down side of compatibility in this area is that off-the-shelf commercial tool management subsystems may already have message formats defined, which would need to be changed.

\subsection{Workbench User Interface}

Within the CIMS environment, the concept of a workbench user interface has been defined. The workbench will provide a single system image to a user by accessing different systems from one terminal. The workbench should also provide common access to different systems by using similar menus and/or function keys. The standard user interface concept is an excellent idea which should be pursued within the tool management system. The user interface for CIMS and TMS should, wherever possible, employ user interface standards that are sound technically and have user/vendor acceptance. 


\section{Bibliography}

ANON., "Good management cuts tooling problems", Metalworking Production, June, 1987, pp. 115-118.

ALBERT, M., "The Vending Machine Approach To Tool Control", Modern Machine Shop, Volume 60, No. 10, March 1988, pp. 51-60.

ALBERT, M., "Tool Management For A Ten-Year-Old FMS", Modern Machine Shop Volume 59, No. 10, March 1987, pp. 54-64.

ATKEY, M., "Only Computers Can Manage...", Machinery and Production Engineering, Volume 144, No. 3685, January 1986, pp. 35-43.

BER, A. and FALKENBURG, D.R., "Tool Management for F.M.S.", Annals of the CIRP, Volume 34, No.1, 1985, pp. 387-391.

BYRKETT, D.L., OZDEN, M.H. and PATTON, J.M., "Integrating Flexible Manufacturing Systems With Traditional Manufacturing, Planning, And Control", Production and Inventory Management Journal, Third Quarter, 1988, pp. 15-20.

CARRIE, A.S. and PERERA, D.T.S., "Work scheduling in FMS under tool availability constraints", International Journal of Production Research, Volume 24, No. 6, 1986, pp. 1299-1308.

COLEMAN, J.R., "Life Extension Through Tool-Condition Monitoring", Machine and Tool Blue Book, March 1987, pp. 46-49.

CONSTANTINIDES, N. and BENNETT, S., "An Investigation Of Methods For The On-Line Estimation Of Tool Wear", International Journal Of Machine Tools \& Manufacture Design, Research And Application, Volume 27, No. 2, 1987, pp. 225-237.

CUPPAN, Dr. B.C., "Tool Management Concerns For Machine Tool Cells and FMS", Cutting Tool Engineering, June, 1987, pp. 51-56.

ELMARAGHY, H. A., "Tooling Rationalization and Automation in Modern Factories", Successful Implementation of Computer-Integrated Manufacturing Conference, Toronto, Ontario, Canada, sponsored by CASA SME, No. MS85-123, 1985.

ELMARAGHY, H.A., "Automated Tool Management in Flexible Manufacturing", Journal of Manufacturing Systems, Volume 4, No. 1, 1985, pp. 1-13. 
GARAPATI, S. and WANG, H.P., "Decision Support System for Tool Management in

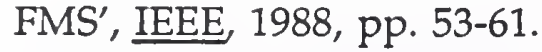

GAYMAN, D. J., "Computers in the Tool Crib", Manufacturing Engineering, Volume 97, No. 3, September 1986, pp. 41-44.

GAYMAN, D.J., "Meeting Production Needs with Tool Management", Manufacturing Engineering, Volume 99, No. 3, September 1987, pp.41-47.

GETTELMAN, K., "Japan's TLC", Modern Machine Shop, Volume 60, No. 7, December 1987, pp. 79-87.

KELLOCK, B., "Vibration Monitoring Signals Tool Failure", Machinery and Production Engineering, Volume 144, No. 3704, November 1986, pp. 19-21.

KELLOCK, B., "Could You Manage To Raise Utilization?", Machinery and Production Engineering, Volume 146, No. 3729, January 1988, pp. 37-44.

KELLOCK, B., "Satisfying The Needs Of An FMS", Machinery and Production Engineering, Volume 144, No. 3685, January 1986, pp. 44-48.

KIRAN, A.S. and KRASON, R.J., "Automating Tooling In A Flexible Manufacturing System", Industrial Engineering, Volume 20, No. 4, April 1988, pp. 52-57.

MARTIN, J.M., "Managing Tools Makes the Cell Click", Manufacturing Engineering, April 1989, pp. 59-62.

MARTIN, M. F., "Tool-ID Systems: One Key to Successful Automation", Machine and Tool Blue Book, March 1987, pp.41-43.

MASON, F., "Computerized Cutting-Tool Management", American Machinist \& Automated Manufacturing, Special Report 786, May 1986, pp. 106-120.

MASON, F., "Tool management in aerospace", American Machinist \& Automated Manufacturing, Volume 131, No. 12, December 1987, pp. 78-81.

MURPHY,K. and CRANFIELD, J.K., "Automatic identification and information systems", The FMS Magazine Volume 5, No. 4, October 1987, pp. 169-172.

PAULICK, R., "Applying toolroom automation", Tooling \& Production, March 1985, pp.94-96. 
PETRONIS, T.J. and KRAUSE, L.R., "Innovative Robotic Workcell Performs Tool Setup", Robotics Today December 1984, pp. 37-39.

PLUTE, M., "Computerized Control Systems For The Tool Crib", Modern Machine Shop, February 1984, pp. 51-58.

POND, J.B. , "Here's Money, In Tool Management", Iron Age, October 3, 1986, pp. $41-43$.

QUINLAN, J.C., "Talking with tools", Tooling \& Production, April, 1988, pp. 50-56.

REUTLINGEN, M.W., "Present Day Tool Organization Manufacturing Technology", Industrial \& Production Engineering, Volume 10, No. 3, 1986, pp. 125-128.

SAMPATH, A. and VAJPAYEE, S., "Tool health monitoring using acoustic emission", International Iournal of Production and Research, Volume 25, No. 5, 1987, pp. 703-719.

SARIN, S.C. and CHEN, C.S., "The machine loading and tool allocation problem in a flexible manufacturing system", International Journal of Production and Research, Volume 25, No. 7, 1987, pp. 1081-1094.

SIEBENTHAL, A., "Tooling for Flexible Manufacturing System", Industrial \& Production Engineering, Volume 10, No. 2, 1986, pp. 63-66.

STAUFFER, R. N., "Tool Handling Advancements", Robotics Today February 1986, pp. 25-27.

SULTANOV, T.A. and BRUK, V.I., "Special Features Of Tooling Organization", Stankri Instrument, Volume 58, Issue 6, 1987, pp. 3-4.

VENTURA, J.A. , CHEN, F.F. and LEONARD, M. S., "Loading Tools To Machines In Flexible Manufacturing Systems", Computers Industry Engineering, Volume 15, Nos. 1-4, 1988, pp. 223-230.

WILDISH, M., "Making Machine Tools Unmanned", Machinery and Production Engineering, Volume 144, No. 3685, January 1986, pp. 30-31.

ZELENY, J., "Flexible Manufacturing Systems with Automatic Transport of Tools", Annals of the CIRP, Volume 30, No. 1, 1981, pp. 349-352. 


\section{Glossary of Terms}

$A G V$

Automated Guided Vehicle.

ASRS

Automated Storage and Retrieval System.

backup tool

(See redundant tool).

$B C L$

Binary Cutter Location data format (EIA Standard RS-494).

buffer storage station (rack)

A buffer storage station (rack) is a table or shelf for storing tool assemblies in the machining cell outside the tool magazines. Tool assemblies are usually picked and placed by a robot.

bulk exchange

A tool allocation strategy. This strategy is to provide a complete dedicated set of tooling for each different part. At the completion of a part run, all tooling would be transferred back to the tool room, to be replaced by a different dedicated set of tools for the next part.

\section{CIMS}

Computer Integrated Manufacturing Systems.

command message

A command message is issued by a supervisory control system to a subordinate control system to tell it what to do. The command message carries work order records which refer to work elements which describe the basic activity the controller is to perform.

computer peripheral

A computer peripheral is a display terminal, a graphics device, a mouse, a printer, a tape unit, etc.

critical path display

A network display of activities and the precedence relationships between those activities. 
cutting conditions

A combination of material hardness, cutter type, cutter coatings, feed rates, and operating/machining parameters that affect tool wear and tool-life.

\section{data acquisition status indication}

An LED or display terminal indication that shows that a data device has completed its read or that a read error exists.

\section{database}

A collection of relations that is managed by a database system.

dispatch list

A list of tasks that are currently ready for processing by a subordinate system or station.

DNC

Distributed Numerical Control

\section{elapsed cutting time}

Time in cut, the machine tool maintains either the accumulated cutting time or decrements a total cutting time to maintain this value.

field

The basic element of database records. This may be a number or a string. It is also referred to as an atomic element.

\section{Freeze Frame}

A modification to the machine tool controller developed at Rock Island Arsenal which allows the real-time capture of cutting parameters, as specified at points in the NC program, or as requested by the operator at the machine tool console.

\section{Gantt chart}

A project management display technique that shows the expected start and completion time, that is the duration for each activity and possibly available slack time.

\section{inventory location report}

An inventory record that shows the current location on the shop floor of an inventory item. 
job

A high level work order, i.e., a work order that is assigned to a system by its supervisor.

job number

The unique identifier that is associated with each job.

job schedule

The expected start and completion times and priorities which in the Rock Island system would be assigned by a higher level system to the manufacturing system on the shop floor.

key

A key is a field which may be used for searching the database to discriminate between records.

\section{$L A N$}

Local Area Network.

machine tool ready station

(See ready station).

machining cell

A set of machining centers that supervised by single control computer and are served by internal material and tool handling systems, sometimes involving a robot.

\section{migration}

A tool allocation strategy. With this strategy there is no complete changeover of tooling. As a job is completed, tools unique to that job are purged from the matrix, and new tools for other jobs are loaded. This strategy allows for reduction of tooling inventory through sharing of common tools between jobs.

network display

(see critical path display).

NIST

National Institute of Standards and Technology. 
operator console

A display terminal where an operator can change the operating mode of a system, i.e., shut it down or bring it up. An individual using an operator console would have more authority to effect system operations than a user with a monitor terminal.

peripheral device

An external computer device such as a terminal, bar code reader, disk drive, printer or plotter.

perishable tooling

Tooling that becomes worn with use and requires replacement or reconditioning,

e.g., drills and mills. Also called consumable tooling.

pixel

A picture element, a single point or dot on a display screen.

process plan

A document specifying the detailed production steps necessary to accomplish a particular job. A typical process plan should have a header, a requirements list, and a procedural steps section.

ready station

A tool exchange point next to a machine tool where the cell tool delivery robot drops off and picks up tool assemblies.

recondition

(See regrind).

regrind

Sharpen a cutting tool.

relation

A set of fields, i.e., a data structure, that makes up a basic record in a database. Relation or table may be used interchangeably.

\section{resident tool}

A tool that has been identified as a high use tool and remains in the machine tool magazine at all times. Resident tools are only replaced when worn and are always replaced by identical tools. 
redundant tool (sister tool)

Multiple, identical setups of the same tool assembly. Redundant tools are required for high use tool assemblies or when tool-life is very short.

resource requirements list

Identifies the tooling materials that are necessary to perform a particular job or task.

rework (pertaining to tool assemblies)

Replacing or indexing worn consumable components of a tool assembly to prepare for immediate reissue of the tool assembly. New preset data is acquired, and the tool assembly is placed back into production.

rework point

A fixed number of minutes of remaining life on a tool at which the tool assembly should be reworked, i.e., inserts should be replaced or indexed or basic high speed steel cutters such as a drill or mill should be replaced.

RIA

Rock Island Arsenal, U.S. Army.

sister tool

(See redundant tool).

status message

A message passed from a subordinate control system to a supervisory system which provides feedback on the state of the subordinate system and its progress in carrying out jobs.

system initialization

The various functions of powering up systems, setting initial data values, performing verification checks, etc.

taper

The conical shaped end of a tool adapter which mates with the machine tool spindle. A number of different standard taper configurations have been defined for tool adapters.

task

A more detailed work order. Many tasks may make up a single job. 
task schedule

The internal schedule of a controller which identifies when each task in a job would be performed and on which system.

TMS

Tool Management System.

tool adapter

The holder into which cutters are placed which mates with the machine tool. Also called a tool holder.

tool allocation

The assignment of individual tool assemblies by tool identification number to specific jobs, tasks or machine tools.

\section{tool allocation plan}

The report which records the assignment of all of the tool assemblies for each job. The tool allocation plan would include tool assembly movement, resident tool assignments, etc.

tool allocation strategy

The rationale, algorithms or logic used to assign tools to specific jobs and/or machines. Some examples include: resident tooling, bulk exchange, migration, and shared tooling.

tool assembly

The combination of tool holder, cutter, extenders, adapters, inserts, etc. that are assembled into a single device that can be placed into a machine tool spindle and used in a manufacturing operation.

tool assembly drawing (tool sketch)

A drawing specification which shows the relationship of various tool components in a tool assembly and the specific dimensions that are required for that assembly.

tool assembly rework

(See rework).

tool availability report

A database report that identifies the number of tools or tooling components that are available in a particular configuration. 
tool components

Inserts, extenders, holders, fasteners, and various individual pieces that make up a tool assembly.

tool identification number

A unique number that is assigned to each tool holder for tracking it as it moves through the system. i.e., A tool identification number is determined by the button or chip mounted on a particular tool holder.

tool-life

The expected cutting time that is available on a tool under a normalized/weighted set of cutting conditions.

tool location report

The current location of all tool assemblies within the system. i.e., at a machine tool, in a storage rack, in the tool room, etc.

tool migration

A technique for allocating tools within a system to make best use of available tooling when and where needed.

tool monitoring

Collecting performance data on cutting tools. Data collected includes torque and thrust values during machining (Freeze Frame system), number of regrinds, tool-life between regrinds, properties of materials machined, physical examination of tools (in process or in tool room); amount of measured flank wear, etc.

tool number ( $R I A$ tool sketch number)

An identifier that has been established at Rock Island to distinguish between different tool assembly setups. Multiple instances of a tool number may exist in a system at one time.

tool offset data

The data that represents the exact length and diameter of a cutting tool as taken at a presetting station.

tool reconditioning

(See regrind). 
tool room exception reports

Reports to the tool management system that expected inventory items were not found or that a tool could not be assembled due to lack of supporting tooling or equipment.

tools

Cutting tools. e.g., drills, mills inserts, taps, etc. In some facilities, tools may also be used to refer to jigs, fixtures, molds, etc.

tool setup

The operations of assembly and presetting tool assemblies to satisfy the tool allocation plan.

tool setup schedule

Identifies when and which tools are to be setup currently and at each scheduled period in the future.

tool setup specifications

A record in the database that includes a drawing which shows the particular setup for the tool number and a components parts list that makes up the tool.

tool setup status

A progress report back to the system that indicates that no action has been taken, the tool has been assembled or the tool has been preset or the tool is ready for issue.

tool sharing

A tool allocation strategy. This strategy recognizes different part pieces share common tooling within a given production period. Common tools are not duplicated. Because tools are only exchanged at the beginning of each production period, all tools must be in the system. At the end of a production period, a completely new set of tooling is loaded into the system.

tool tracking

The process of recording each movement of the tool assembly through the system so that it can be immediately retrieved at any time for other usage.

tool usage profile

A list of all jobs that a specific tool is used on and the number of minutes of cutting life spent by the tool on each particular job. 
virtual storage

All areas of the tool management system are considered valid tool assembly storage locations, e.g., tool room, transport device, machine tool magazines, storage racks in the cell, etc. Tool assemblies are tracked at all times so they can be immediately retrieved for use. Tool assemblies are not, for example, removed from a machine tool magazine because they are not required for the next job. The machine tool magazine is a valid storage location as long as the space is not required for a different tool at that machine.

wand

A hand held device that is used to read tool identification micro-chips. "To wand" is to read the micro-chip device. 
1. PUBUCATION OR REPORT NUMBER

\section{BIBLIOGRAPHIC DATA SHEET}

\section{TITLE AND SUBTITLE}

System Requirements Analysis for the U.S. Army Rock Island Arsenal Tool Management System

5. AUTHOR(S)

Simon P. Frechette, Charles R. McLean

i. PERFORMING ORGANIZATION (IF JOINT OR OTHER THAN NIST, SEE INSTRUCTIONS)

U.S. DEPARTMENT OF COMMERCE

NATIONAL INSTITUTE OF STANDARDS AND TECHNOLOGY

GATTHERSBURG, MD 20899

7. CONTRACT/GRANT NUMBER

8. TYPE OF REPORT AND PERIOD COVERED Final

3. SPONSORING ORQANIZATION NAME AND COMPLETE ADDRESS (STREET, CITY, STATE, ZIP)

U.S. Army Rock Island Arsenal

Rock Island, IL 61299-6000

10. SUPPLEMENTARY NOTES

DOCUMENT DESCRIBES A COMPUTER PROGRAM; SF-185, FIPS SOFTWARE SUMMARY, IS ATTACHED.

11. ABSTRACT (A 200-WORD OR LESS FACTUAL SUMMARY OF MOST SIGNIFICANT INFORMATION. IF DOCUMENT INCLUDES A SIGNIFICANT BIBUOORAPHY OR UTERATURE SURVEY, MENTION IT MERE,

The U.S. Army is conducting an extensive manufacturing modernization program at the Rock Island Arsenal (RIA) in Illinois. Tool management, as it applies to these automated systems, is one area that RIA has identified for additional modernization activities. Engineers at the Rock Island Arsenal and the National Institute of Standards and Technology (NIST) recognize that a consistent and integrated implementation of tool management is critical to the Army's manufacturing program.

The purpose of this document is to outline requirements for implementing an-automated tool management system at Rock Island. Tool management is not a single, simple activity-it is comprised of a complex and pervasive set of functions. No comercial systems are known to exist which perform all of the functions outlined in this document. This study documents joint efforts by NIST and RIA staff to develop an open architecture for tool management at the Rock Island manufacturing facility.

12. KEY WORDS (6 TO 12 ENTRIES; ALPHABETICAL ORDER; CAPITALZE ONLY PMOPEA MAMES; AND SEPARATE KEY WORDS BY SEMICOLONS)

tool management; tool allocation; tool 1ife; Rock Island Arsenal; machining cell; tool identification; tool migration

\section{AVAILABISTYY}

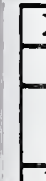

UNUMITED

FOR OFFCLAL DISTRIBUTION. DO NOT RELEASE TO NATIONAL TECHNICAL INFORMATION SERVICE (NTIS).

ORDER FROM SUPERINTENDENT OF DOCUMENTS, U.S. GOVERNMENT PRINTING OFFICE, WASHINGTON, DC 20402

ORDER FROM MATIONAL TECHNICAL INFORMATION SERVICE (NTIS), SPRINGFIELD, VA 22161.
14. MUMBEA OF PRINTED PAGES

15. PRICE A06 


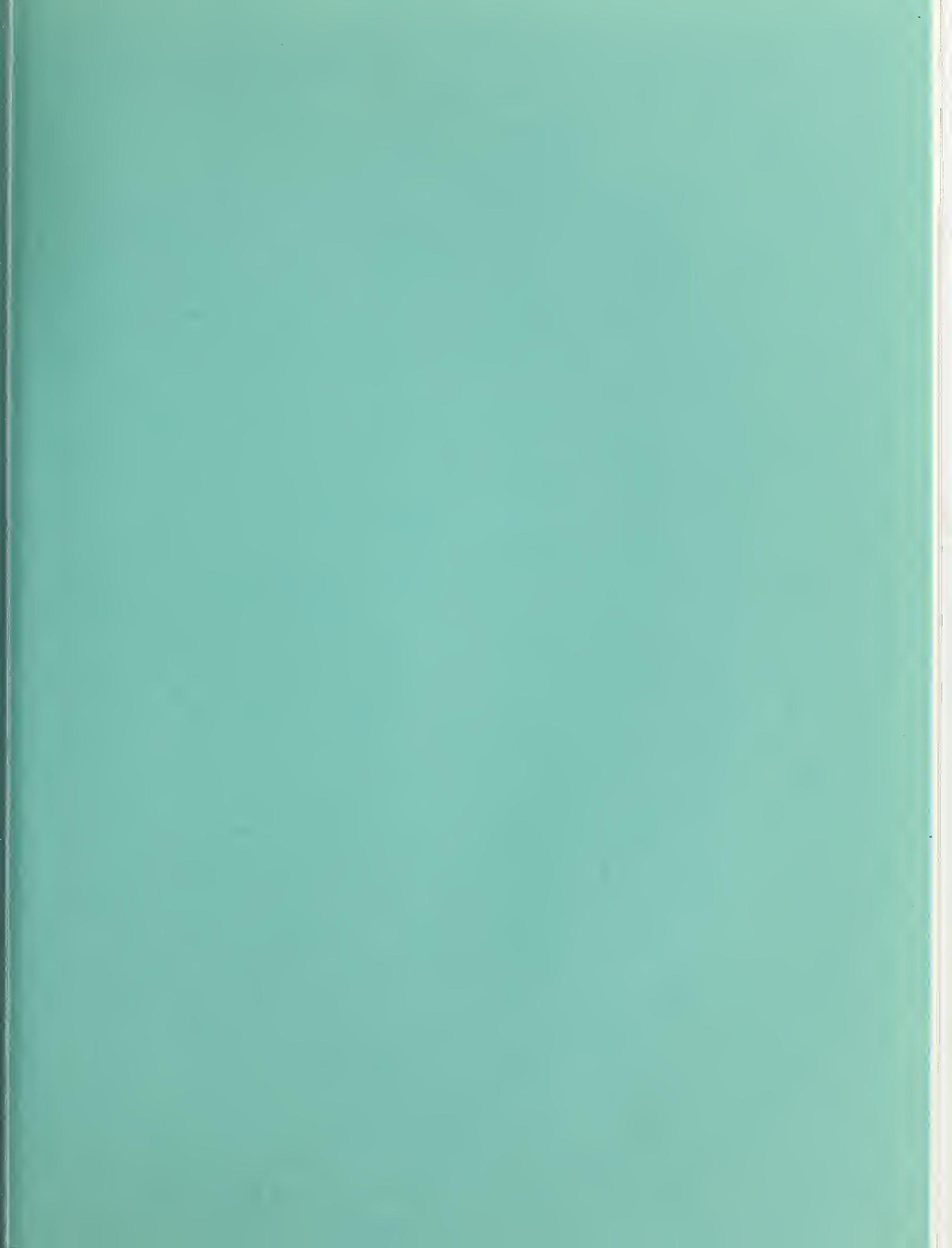
